\begin{abstract}
Kharebava, Zviad. Singular Cochains and Rational Homotopy Type. (Thesis Director: Dr. Tom Lada)

Rational homotopy types of simply connected topological spaces have been classified by weak equivalence classes of commutative cochain algebras (Sullivan) and by isomorphism classes of minimal commutative $A_{\infty}$-algebras (Kadeishvili).

We classify rational homotopy types of the space $X$ by using the (non-commutative) singular cochain complex, $C^{*}(X, Q)$, with additional structure given by the homotopies introduced by Baues, $\left\{E_{1, k}\right\}$ and $\left\{F_{p, q}\right\}$. We show that if we modify the resulting $B_{\infty}$-algebra structure on this algebra by requiring that its bar construction be a Hopf algebra up to homotopy, then weak equivalence classes of such algebras classify rational homotopy types.
\end{abstract}




\title{
Singular Cochains and Rational Homotopy Type
}

\author{
by \\ Zviad Kharebava \\ Bachelor of Science, Georgian Technical University, Tbilisi, 1995 \\ Master of Science, Georgian Technical University, Tbilisi, 1998
}

Submitted in Partial Fulfillment of the Requirements

for the Degree of Doctor of Philosophy in the

Department of Mathematics

North Carolina State University

2004

Dr. Tom Lada

Chair, Advisory Committee

Dr. Amassa Fauntleroy

Committee Member
Dr. Ernie Stitzinger

Committee Member

Dr. Kailash Misra

Committee Member 


\section{BIOGRAPHY}

The author was born in Republic of Georgia in 1973. He received Bachelor of Science in mathematics, 1995, and Master of Science in mathematics, 1998, from the Georgian Technical University, Tbilisi.

During the years 1998-2000 he was an aspirant at A.Razmadze Mathematical Institute of the Georgian Academy of Sciences.

In 2001 he entered the graduate program in mathematics at North Carolina State University. 


\section{ACKNowledGements}

I want to thank my supervisor, Dr. Tom Lada, for his helpful guidance throughout the dissertation.

Also I want to thank Professor James Stasheff, Professor Tornike Kadeishvili, and

Professor Samson Saneblidze for their useful comments and suggestions on my work.

Finally, I want to thank the entire Mathematics Department of North Carolina State University for making my stay at NC State memorable. 


\section{Contents}

Introduction 1

1. Review of notation and known results 4

1.1 DG module 4

1.2 DG algebra 4

1.3 DG coalgebra 5

1.4 DG Hopf algebra $\quad 6$

$\begin{array}{ll}1.5 \text { Suspension } & 7\end{array}$

$\begin{array}{ll}1.6 \text { Tensor algebra } & 7\end{array}$

1.7 Tensor coalgebra 8

$\begin{array}{ll}1.8 \text { Lie algebra } & 10\end{array}$

1.9 Lie coalgebra $\quad 15$

$1.10 A_{\infty}$-algebra $\quad 19$

1.11 Twisting cochain 22

1.12 Perturbation Lemma 26

1.13 Hopf algebras up to homotopy 31

1.14 Homotopy G-algebra 39

2. On the Gugenheim map 45

2.1 Sullivan functor and some key lemmas 46

2.2 Multiplicativity up to homotopy $\quad 57$

3. $\tilde{B}_{\infty}$-algebra category $\quad 59$

$\begin{array}{lll}3.1 B_{\infty} \text {-algebra } & 59\end{array}$ 
$3.2 A_{\infty}$-algebra structure in homology

4. Rational homotopy type

Bibliography 


\section{INTRODUCTION}

Throughout the paper we work over the rationals $Q$, unless otherwise specified.

The singular cochain algebra $C^{*}(X, Q)$ of a topological space $X$ almost never is commutative, although it is homotopy commutative. However, we can replace $C^{*}(X, Q)$ by a genuinely commutative cochain algebra. More precisely, there is a commutative cochain algebra $A_{P L}(X, Q)[43]$ and natural cochain algebra quasiisomorphisms

$$
C^{*}(X, Q) \stackrel{\simeq}{\longrightarrow} D(X) \stackrel{\simeq}{\longleftarrow} A_{P L}(X, Q)
$$

where $D(X)$ is a third natural cochain algebra. The contravariant functor $X \longmapsto$ $A_{P L}(X, Q)$ serves as the fundamental bridge to transfer problems from topology to algebra.

Quasi-isomorphisms above define a natural isomorphism of graded algebras

$$
H^{*}(X, Q)=H\left(A_{P L}(X, Q)\right)
$$

through which they are always identified; thus for any continues map, $f$, we identify $H^{*}(f, Q)=H\left(A_{P L}(f, Q)\right)$.

Recall that simply connected spaces $X$ and $Y$ have the same rational homotopy type if and only if there is a chain of rational homotopy equivalences

$$
X \rightarrow Z_{0} \leftarrow Z_{1} \rightarrow Z_{2} \leftarrow \cdots \rightarrow Z_{k} \leftarrow Y
$$

(a map $f: W \rightarrow Z$ is a rational homotopy equivalence if $H^{*}(f, Q)$ is an isomorphism). There is an analogous notion for commutative cochain algebras: two commutative cochain algebras $(A, d)$ and $(B, d)$ are weakly equivalent if they are connected by the 
chain

$$
(A, d) \stackrel{\simeq}{\longleftarrow}(C(0), d) \stackrel{\simeq}{\longrightarrow}(C(1), d) \stackrel{\simeq}{\longleftarrow} \cdots \stackrel{\simeq}{\longleftarrow}(C(k), d) \stackrel{\simeq}{\longrightarrow}(B, d)
$$

of quasi-isomorphisms of commutative cochain algebras; such a chain is called a weak equivalence between $(A, d)$ and $(B, d)$.

It follows that if $X$ and $Y$ have the same rational homotopy type then $A_{P L}(X, Q)$ and $A_{P L}(Y, Q)$ are weakly equivalent; and so $A_{P L}(-, Q)$ defines a map

$$
\begin{aligned}
\{\text { rational homotopy types }\} \rightarrow & \text { \{weak equivalence classes of } \\
& \text { commutative cochain algebras }\}
\end{aligned}
$$

Moreover, it was shown in [43] that if simply connected spaces $X$ and commutative cochain algebras $(A, d)$ are such that $H^{*}(X, Q)$ and $H(A, d)$ have finite type, then this correspondence is a bijection.

Later Kadeishvili described the rational homotopy types in terms of $A_{\infty}$-algebras; it was shown that $X$ and $Y$ have the same rational homotopy type if and only if $H\left(A_{P L}(X, Q)\right)$ and $H\left(A_{P L}(Y, Q)\right)$ are isomorphic commutative $A_{\infty}$-algebras [25]; this relation defines a map

$$
\begin{aligned}
\{\text { rational homotopy types }\} \rightarrow & \text { isomorphism classes of minimal } \\
& \text { commutative } \left.A_{\infty} \text {-algebras }\right\}
\end{aligned}
$$

which turns out to be a bijection if simply connected spaces $X$ and commutative $A_{\infty}$-algebras $\left(A,\left\{m_{i}\right\}\right)$ are such that $H^{*}(X, Q)$ and $H(A)$ are of finite type, see [25].

In both cases above the bijections were established using Sullivan's commutative $D G$-algebra $A_{P L}(X, Q)$. In our dissertation, we classify the rational homotopy types of simply connected space $X$ by using the (noncommutative) singular cochain algebra, $\left(C^{*}(X, Q), d_{C^{*}(X, Q)}, \smile\right)$, with additional structure given by the homotopies

$$
\left\{E_{1, k}: C^{*}(X, Q) \otimes\left(C^{*}(X, Q)^{\otimes k}\right) \rightarrow C^{*}(X, Q)\right\}
$$


with $E_{1,1}=\smile_{1}$ and

$$
\left\{F_{p, q}:\left(C^{*}(X, Q)^{\otimes p}\right) \otimes\left(C^{*}(X, Q)^{\otimes q}\right) \rightarrow C^{*}(X, Q)\right\}
$$

with $F_{1,1}=\smile_{2}$ of Baues [6], [7].

In Section 3 we introduce the notion of $\tilde{B}_{\infty^{-}}$-algebra, a modified version of $B_{\infty^{-}}$ algebra of Getzler and Jones [19], and prove the following

Theorem 74 The 1-reduced spaces $X$ and $Y$ have the same rational homotopy type if and only if $\left(C^{*}(X), d_{C^{*}(X)}, \smile,\left\{E_{1, k}^{X}\right\},\left\{F_{p, q}^{X}\right\}\right)$ and $\left(C^{*}(Y), d_{C^{*}(Y)}, \smile,\left\{E_{1, k}^{Y}\right\},\left\{F_{p, q}^{Y}\right\}\right)$ are weakly equivalent in the $\tilde{B}_{\infty}$-algebra category.

Finally we show that the singular cochain functor $C^{*}$ induces an equivalence of rational homotopy categories

$$
\mathcal{H} o C^{*}: \mathcal{H o C W} W_{1} \rightarrow \mathcal{H} o \tilde{B}_{\infty}-A l g
$$

see Theorems 75 and 76. 


\section{REVIEW OF NOTATION AND KNOWN RESUltS}

In this section we collect the definitions and facts we need; most can be found in $[\mathbf{3}]$, [25] and [41]. Since these results are not new, we omit their proofs.

\subsection{DG MODULE}

Let $R$ be a commutative ring with identity $1_{R}$. Let $\left\{M_{i}\right\}_{i \in \mathbb{Z}}$ be a sequence of $R$ -

modules; $M=\sum M_{i}$ is a called a graded $R$-module (throughout this paper we assume $M_{i}=0$ for $i<0$ ). An element $x \in M_{i}$ is said to be homogeneous of degree $i$, in which case we write $|x|=i$.

A differential graded module ( $D G$-Modules) is a graded module $M$ together with an endomorphism $d \in \operatorname{End}(M)$ of degree +1 and square zero. Given $D G$-Modules $M, N$ and a homomorphism $f:\left(M, d_{M}\right) \rightarrow\left(N, d_{N}\right)$ of degree $k$, define the differential of $f$ by $D f=d_{N} f-(-1)^{k} f d_{M}$. The category of $D G$-Modules is denoted by $D G M$; its morphisms are chain maps, i.e., maps $f$ of degree 0 with $D f=0$.

A $D G M$ homomorphism inducing an isomorphism on homology is called a quasiisomorphism.

\subsection{DG ALGEBRA}

$D G A$ denotes the category of augmented associative $D G$-algebras with unit. If $(A, d, \mu, \eta, \varepsilon) \in D G A$, then the multiplication $\mu: A \otimes A \rightarrow A$, the unit $\eta: R \rightarrow A$, 
the augmentation $\varepsilon: A \rightarrow R$ and the differential $d: A \rightarrow A$ satisfy the usual requirements. We say that $A$ is connected, if $\eta: R \rightarrow A_{0}$ is an isomorphism. To simplify notation we omit $\eta$ and $\varepsilon$ and write $(A, d, \mu)$ for $(A, d, \mu, \eta, \varepsilon)$.

A morphism $f: A \rightarrow B$ in $D G A$ is a chain map preserving all structure. A $D G A$ homomorphism inducing an isomorphism on homology is called quasi-isomorphism.

Let $I A=\operatorname{ker}(\varepsilon)$ be the augmentation ideal of $A$. Then the exact sequence $0 \rightarrow$

$I A \stackrel{i}{\rightarrow} A \stackrel{\varepsilon}{\rightarrow} R \rightarrow 0$ defines a functor $I: D G A \rightarrow D G M$; the multiplication $\mu$ induces a map $I \mu: I A \otimes I A \rightarrow I A$ given by $i(I \mu)=\mu(i \otimes i)$.

Let $f, g:\left(A, d_{A}, \mu_{A}\right) \rightarrow\left(B, d_{B}, \mu_{B}\right) \in D G A$. A degree $r R$-homomorphism $F: A \rightarrow B$ is an $(f, g)$-derivation if it satisfies

$$
F \mu_{A}=\mu_{B}\left(F \otimes g+(-1)^{r} f \otimes F\right)
$$

A derivation homotopy from $f$ to $g$ is an $(f, g)$-derivation of degree +1 further satisfying $f-g=d_{B} F+F d_{A}$.

We call the DG-algebra $(A, d, \mu) 1$-connected if it is connected and $A_{1}=0$.

\subsection{DG COALGEBRA}

$D G C$ denotes the category of coaugmented differential graded coassociative coalgebras ( $D G$ Coalgebras) with counit. If $(C, d, \Delta, \eta, \varepsilon) \in D G C$, the comultiplication $\Delta: C \rightarrow C \otimes C$, the counit $\varepsilon: C \rightarrow R$, the coaugmentation $\eta: R \rightarrow C$ and the differential $d: C \rightarrow C$ satisfy the usual requirements. We say that $C$ is connected, if $\varepsilon: C_{0} \rightarrow R$ is an isomorphism. To simplify notation we omit $\eta$ and $\varepsilon$ and write $(C, d, \Delta)$ for $(C, d, \Delta, \eta, \varepsilon)$.

A morphism $f: C \rightarrow D$ in $D G C$ is a chain map preserving all structure. A $D G C$ homomorphism inducing an isomorphism on cohomology is called quasi-isomorphism. 
Let $J C=c o \operatorname{ker}(\eta)$ be the coaugmentation ideal of $C$. Then the exact sequence $0 \rightarrow R \stackrel{\eta}{\rightarrow} C \stackrel{p}{\rightarrow} J C \rightarrow 0$ defines a functor $J: D G C \rightarrow D G M$; the comultiplication $\Delta$ induces a map $J \Delta: J C \rightarrow J C \otimes J C$ given by $(J \Delta) p=(p \otimes p) \Delta$.

Let $f, g:\left(C, d_{C}, \Delta_{A}\right) \rightarrow\left(D, d_{D}, \Delta_{D}\right) \in D G C$. A degree $r R$-homomorphism $F: C \rightarrow D$ is an $(f, g)$-coderivation if it satisfies

$$
\Delta_{D} F=\left(F \otimes g+(-1)^{r} f \otimes F\right) \Delta_{C} .
$$

A coderivation homotopy from $f$ to $g$ is an $(f, g)$-coderivation of degree +1 further satisfying $f-g=d_{D} F+F d_{C}$.

We call the DG-coalgebra $(C, d, \Delta) 1$-connected if it is connected and $C_{1}=0$.

\subsection{DG Hopf ALGEBRA}

A connected differential graded Hopf algebra (DGHA) is defined to be a quartet $(A, d, \mu, \Delta)$ such that $(A, d, \mu)$ is a connected differential graded algebra, $(A, d, \Delta)$ is a connected differential graded coalgebra and $\Delta \mu=(\mu \otimes \mu)(1 \otimes T \otimes 1)(\Delta \otimes \Delta)$. Notice that the last equality is equivalent to saying either that

$$
\Delta: A \rightarrow A \otimes A,
$$

is a morphism of algebras or that

$$
\mu: A \otimes A \rightarrow A
$$

is a morphism of coalgebras. The $D G H A A$ is cocommutative if $T \Delta=\Delta$ and commutative if $\mu T=\mu$, where $T: A \otimes A \rightarrow A \otimes A$ is the twisting involution given by $T(a \otimes b)=(-1)^{|a||b|} b \otimes a$.

A morphism $f:(A, d, \mu, \Delta) \rightarrow\left(A^{\prime}, d^{\prime}, \mu^{\prime}, \Delta^{\prime}\right)$ between $D G$ Hopf algebras is defined to be a graded $R$-module homomorphism compatible with the both structures; i.e. $d^{\prime} f=f d, f \mu=\mu^{\prime}(f \otimes f)$ and $\Delta^{\prime} f=(f \otimes f) \Delta$. 
It is easy to check that connected $D G$ Hopf algebras and their morphisms form a category.

\subsection{Suspension}

The suspension functor $s^{r}: D M \rightarrow D M$ is given by $\left(s^{r} M\right)_{n}=M_{n-r}$ and $d_{s^{r} M}=$ $-d_{M}$. On an object $M$, the map $s^{r}: M \rightarrow s^{r} M$ has degree $r$, is the identity in each dimension and $D s=0$. If $f: M \rightarrow N$ has degree $k$, so does $s^{r} f: s^{r} M \rightarrow s^{r} N$ and the following diagram commutes:

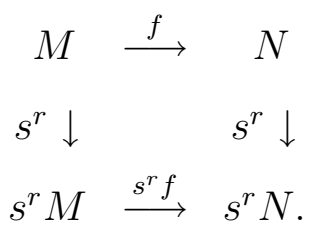

\subsection{Tensor Algebra}

Let $M$ be a graded R-module with $M_{0}=0$.

A tensor algebra $T(M)$ on a graded module $M$ is the direct sum $T(M)=$ $\sum_{i \geq 0} M^{\otimes i}$, where $M^{\otimes 0}=R$, with multiplication given by

$$
\mu\left(\left(a_{1} \cdots a_{i}\right) \otimes\left(a_{i+1} \cdots a_{n}\right)\right)=\left(a_{1} \cdots a_{n}\right) .
$$

Let $i_{1}: M \rightarrow T(M)$ be the injection. $T(M)$ has the following universal property: If $A$ is any connected DGA and $\alpha: M \rightarrow I A$ is any homomorphism, there is a unique algebra map $f_{\alpha}: T(M) \rightarrow A$ which makes the following diagram commute:

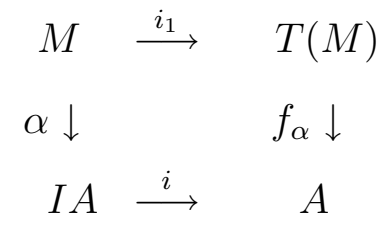


An explicit formula for $f_{\alpha}$ is given by

$$
f_{\alpha}=\mu_{A}^{n}\left(\alpha^{\otimes n} \otimes i^{\otimes n}\right)
$$

where $\mu_{A}^{1}=I d, \mu_{A}^{2}=\mu_{A}$ and $\mu_{A}^{n}=\mu_{A}\left(\mu_{A}^{n-1} \otimes I d\right)$.

The tensor algebra $T(M)$ has the analogous universal property with respect to derivations: for any graded $T(M)$-module $A$ and for any homomorphism $\beta: M \rightarrow I A$ there exists a derivation $D_{\beta}: T(M) \rightarrow A$ such that $D_{\beta} i_{1}=\beta$.

Let $(C, d, \triangle)$ be a connected DGC. The Cobar construction $\Omega(C, d, \triangle)$ due to Adams [2] is defined as a free tensor algebra $T\left(s^{-1}(J C)\right)$ whose differential $d_{\Omega}$ is defined on generators $s^{-1} J C$ by

$$
d_{\Omega} \cdot i^{1}=-i^{1}\left(s^{-1} d s\right)+i^{2}\left(s^{-1} \otimes s^{-1}\right)(J \Delta) s
$$

where $i^{k}:\left(s^{-1} J C\right)^{\otimes k} \rightarrow T\left(s^{-1} J C\right)$ is the obvious inclusion.

Thus the Cobar construction of a 1-connected DGC is a connected DGA with augmentation $1: R \rightarrow R$.

\subsection{Tensor COAlgebra}

Let $M$ be a graded R-module with $M_{0}=0$.

A tensor coalgebra $T^{c}(M)$ on a graded module $M$ is the direct sum $T^{c}(M)=$ $\sum_{i \geq 0} M^{\otimes i}$, where $M^{\otimes 0}=R$, with diagonal given by

$$
\Delta\left(a_{1} \cdots a_{n}\right)=\sum_{i=0}^{n}\left(a_{1} \cdots a_{i}\right) \otimes\left(a_{i+1} \cdots a_{n}\right) .
$$

Let $P_{1}: T^{c}(M) \rightarrow M$ be the projection. $T^{c}(M)$ has the following universal property: If $C$ is a any connected DGC and $\alpha: J C \rightarrow M$ is any homomorphism, there is a unique coalgebra map $f_{\alpha}: C \rightarrow T^{c}(M)$ which makes the following diagram 
commute:

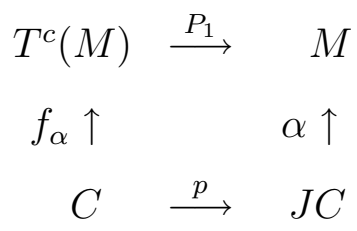

An explicit formula for $f_{\alpha}$ is given by

$$
f_{\alpha}=\sum_{i=1}^{\infty}\left(p^{\otimes i} \otimes \alpha^{\otimes i}\right) \Delta_{C}^{i},
$$

where $\Delta_{C}^{1}=I d, \Delta_{C}^{2}=\Delta_{C}$ and $\Delta_{C}^{i}=\left(\Delta_{C}^{i-1} \otimes I d\right) \Delta_{C}$.

The tensor coalgebra $T^{c}(M)$ has the analogous universal property with respect to coderivations: for any graded $T^{c}(M)$-comodule $C$ and for any homomorphism $\beta: C \rightarrow M$ there exists a coderivation $D_{\beta}: C \rightarrow T^{c}(M)$ such that $P_{1} D_{\beta}=\beta$.

Let $(A, d, \mu)$ be a connected DGA. The Bar construction $B(A, d, \mu)$ is the DGC $T^{c}(s(I A))$ with differential (coderivation)

$$
\begin{array}{r}
d_{B A}\left[a_{1}|\cdots| a_{n}\right]=\sum_{i=0}^{n-1}\left[\bar{a}_{1}|\cdots| \bar{a}_{i}\left|d a_{i+1}\right| a_{i+1}|\cdots| a_{n}\right] \\
\quad+\sum_{i=0}^{n-2}\left[\bar{a}_{1}|\cdots| \bar{a}_{i}\left|\mu\left(\bar{a}_{i+1} \otimes a_{i+1}\right)\right| a_{i+k+1}|\cdots| a_{n}\right],
\end{array}
$$

where $\bar{a}=(-1)^{|a|+1} a$, and the degree of $\left[a_{1}|\cdots| a_{n}\right] \in B A$ is $\sum_{i=1}^{n}\left|a_{i}\right|+n$.

The Bar construction of a 1 -connected $D G A$ is a connected $D G C$ with coaugmentation $1: R \rightarrow R$.

Let $S_{p, q}$ denote the shuffle permutations in the set of all permutations $\sum_{p+q}$, i.e., $\sigma \in S_{p, q}$ implies $\sigma(1)<\cdots<\sigma(p), \sigma(p+1)<\cdots<\sigma(p+q)$. The shuffle product $\mu_{s h}: B A \otimes B A \rightarrow B A$ is given by

$$
\mu_{s h}\left(\left[a_{1}|\cdots| a_{p}\right] \otimes\left[a_{p+1}|\cdots| a_{p+q}\right]\right)=\sum_{\sigma \in S_{p, q}} \varepsilon(\sigma)\left[a_{\sigma^{-1}(1)}|\cdots| a_{\sigma^{-1}(p+q)}\right]
$$

where $\varepsilon(\sigma)=+1$ if the number of transpositions is even, and $\varepsilon(\sigma)=-1$ if the number of transpositions is odd. If $\operatorname{DGA}(A, d, \mu, \eta, \varepsilon)$ is commutative, then the 
differential $d_{B A}: B A \rightarrow B A$ on the Bar construction is a derivation of $\mu_{s h}$ in which case $\left(B A, \Delta, d_{m}, \mu_{s h}\right)$ is a DGHA.

\subsection{LIE ALGEBRA}

In the following two sections we give the main definitions and facts about Lie algebras and Lie coalgebras. References include $[\mathbf{1}],[\mathbf{3}],[\mathbf{8}],[\mathbf{2 5}],[\mathbf{3 5}]$. For simplicity we consider vector spaces over the field of characteristic 0 .

Recall that a Lie algebra consists of a vector space $L$ together with a linear map $[]:, L \otimes L \rightarrow L$ (called the bracket) such that

$$
\begin{aligned}
& {[x, x]=0 \quad x \in L} \\
& {[x,[y, z]]+[x,[y, z]]+[x,[y, z]]=0 \quad x, y, z \in L .}
\end{aligned}
$$

In this form, the definition of Lie algebra is not dualizable: what we must to do is

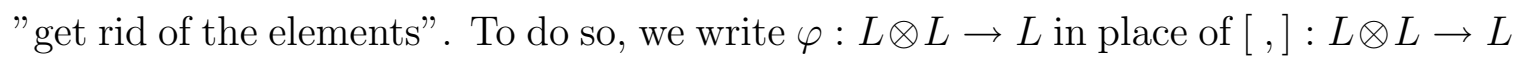
and

$$
\xi: L \otimes L \otimes L \rightarrow L \otimes L \otimes L
$$

for the linear map induced by the map

$$
L \times L \times L \rightarrow L \times L \times L
$$

which cyclically permutes the co-ordinates (i.e. $(x, y, z) \mapsto(y, z, x))$. In effect,

$$
\xi=\xi_{V}: V \otimes V \otimes V \rightarrow V \otimes V \otimes V
$$

may be defined for any vector space $V$ as the composite

$$
V \otimes V \otimes V \stackrel{\simeq}{\rightarrow} V \otimes(V \otimes V) \stackrel{T_{V \otimes(V \otimes V)}}{\longrightarrow}(V \otimes V) \otimes V \stackrel{\simeq}{\rightarrow} V \otimes V \otimes V .
$$

In this notation Jacobi identity becomes

$$
\varphi\left(1_{L} \otimes \varphi\right)\left(1_{L \otimes L \otimes L}+\xi_{L}+\xi_{L}^{2}\right)=0
$$


and the (1) condition can be replaced by

$$
\operatorname{ker}(1-T) \subset \operatorname{ker}(\varphi)
$$

Definition 1. A graded Lie algebra over $R$ is a pair $(L, \varphi)$, where $L$ is a graded vector space and $\xi: L \otimes L \rightarrow L$ is a linear map subject to the conditions

$$
\begin{aligned}
& \text { (1) } \quad \operatorname{ker}(1-T) \subset \operatorname{ker}(\varphi) \\
& \text { (2) } \varphi(1 \otimes \varphi)\left(1+\xi+\xi^{2}\right)=0 .
\end{aligned}
$$

A graded Lie algebra $L$ is called $t$-reduced if $L_{n}=0$ for $n<t$; a 1-reduced Lie algebra is also called connected.

Definition 2. A subspace $V$ is called a Lie subalgebra of a Lie algebra $L$ if $\varphi(V \otimes V) \subset V$

We give here two familiar examples of a Lie algebra which we will use in our further definitions:

Example (1): Let $(A, \mu)$ be a connected associative algebra, then $(I A, I \mu-I \mu T)$ is a Lie algebra denoted by $l(A)$. Recall that by $I A$ we denoted an augmentation ideal of algebra $A$, Subsection 1.2.

Example (2): Let $(H, \mu, \Delta)$ be a connected Hopf algebra with associative multiplication. Then $P(H)$, the space of primitives of $H$, carries a structure of a Lie algebra. Recall that $P(H)$ is the space

$$
\{x \in H \mid \Delta x=1 \otimes x+x \otimes 1\} .
$$

Since the diagonal $\Delta$ of $(H, \mu, \Delta)$ is an algebra map, we easily find that

$$
\Delta[x, y]=1 \otimes[x, y]+[x, y] \otimes 1
$$

where $x, y \in P H$ and $[x, y]=\mu(x \otimes y)-\mu T(x \otimes y)$. As we can see $P H$ is a Lie subalgebra of $l(H)$. 
Definition 3. A homomorphism $f:\left(L_{1}, \varphi_{1}\right) \rightarrow\left(L_{2}, \varphi_{2}\right)$ is called a Lie algebra homomorphism if $f \varphi_{1}=\varphi_{2}(f \otimes f)$.

The category of Lie algebras and Lie algebra homomorphisms is denoted by $L A$.

Let $V$ be a vector space with $V_{0}=0$, and $\left(T V, \mu, \Delta_{s h}\right)$ be a connected Hopf algebra over $V$.

DEFINITION 4. The free Lie algebra over vector space $V$ is defined as the space of primitives $P\left(T V, \mu, \Delta_{s h}\right)$.

The free Lie algebra over vector space $V$ is denoted by $\mathcal{L}(V)$.

From Subsection 1.6 we know that any graded algebra map $f: T(V) \rightarrow A$, where $A$ is a connected graded algebra, is uniquely defined by its restriction on generators $\alpha=\left.f\right|_{V}: V \rightarrow I A$ and $f=f_{\alpha}$. Let $(A, m, \Delta)$ be any connected cocommutative graded Hopf algebra and

$$
f_{\alpha}:(T V, \mu) \rightarrow(A, m)
$$

any graded algebra homomorphism. Then $f_{\alpha}:\left(T(V), \mu, \Delta_{s h}\right) \rightarrow(A, m, \Delta)$ is a graded Hopf algebra homomorphism if and only if $\operatorname{Im}(\alpha) \subseteq P A$, i.e. $\alpha(v)$ is a primitive for any $v \in V$.

We also know that any derivation $D: T V \rightarrow T V$ is uniquely defined by its restrictions on generators $\beta=\left.D\right|_{V}: V \rightarrow I(T V)$ and $D=D_{\beta}$. Let

$$
D_{\beta}:(T V, \mu) \rightarrow(T V, \mu)
$$

be any derivation. Then $D_{\beta}$ is also coderivation with respect to $\Delta_{s h}$ (i.e. $D_{\beta}$ is a Hopf derivation) if and only if $\operatorname{Im}(\beta) \subseteq P T(V)=\mathcal{L}(V)$, i.e. $\beta(v)$ is a primitive for any $v \in V$.

The free Lie algebra $\mathcal{L}(V)$ has the analogous universal property as the tensor algebra $T(V)$ : if $L$ is any connected graded Lie algebra and $\alpha: V \rightarrow L$ is any homomorphism, then there is a unique Lie algebra map $f_{\alpha}: \mathcal{L}(V) \rightarrow L$ which makes 
the following diagram commute:

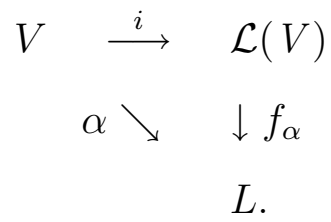

DeFinition 5. The universal enveloping algebra $U L$ of a Lie algebra $(L, \varphi)$ is an object in DGA together with a morphism

$$
i_{U L}: L \rightarrow l(U L)
$$

in $L A$, such that for any $A \in D G A$ and any morphism $f: L \rightarrow l(A)$ in $L A$, there exists a unique morphism $F: U L \rightarrow A$ of $D G A$ making the diagram

$$
\begin{array}{r}
L \stackrel{i_{U L}}{\longrightarrow} l(U L) \\
f \searrow \downarrow l(F)
\end{array}
$$

commutative.

Let's look at the construction of $U L$; let $(T L, \mu)$ be a tensor algebra and $I$ be a smallest ideal of $T L$ containing $\operatorname{Im}\left\{\mu(1-T)\left(i_{T L} \otimes i_{T L}\right)-i_{T L} \varphi\right\}$. Then $U L=\frac{T L}{I}$ and $i_{U L}=P i_{T L}$, where $i_{T L}: L \rightarrow T L$ and $P: T L \rightarrow U L=\frac{T L}{I}$ are the natural inclusion and the natural projection correspondingly.

In fact, $U L$ inherits from $\left(T L, \mu, \Delta_{s h}\right)$ a coalgebra structure too. We mention once and for all that $\Delta_{U L}$ makes $U L$ into cocommutative coassociative Hopf algebra; moreover, there is a natural Hopf algebra isomorphism

$$
U \mathcal{L}(\mathrm{V})=\mathrm{TV}
$$

$($ see $[36])$.

A differential graded Lie algebra is a connected graded Lie algebra $(L, \varphi)$ together with a derivation $\delta$, i.e. $\delta \varphi=\varphi(\delta \otimes 1-1 \otimes \delta)$, of degree -1 whose square is 0 . 
A differential graded Lie algebra homomorphism

$$
f:\left(L_{1}, \varphi_{1}, \delta_{1}\right) \rightarrow\left(L_{2}, \varphi_{2}, \delta_{2}\right)
$$

is a graded Lie algebra homomorphism $f:\left(L_{1}, \varphi_{1}\right) \rightarrow\left(L_{2}, \varphi_{2}\right)$ with $f \delta=\delta f$.

The free Lie algebra $\mathcal{L}(V)$ has the analogous universal property with respect to derivations: for any homomorphism $\beta: V \rightarrow \mathcal{L}(V)$ there is a unique derivation $D_{\beta}: \mathcal{L}(V) \rightarrow \mathcal{L}(V)$ which makes the following diagram commute:

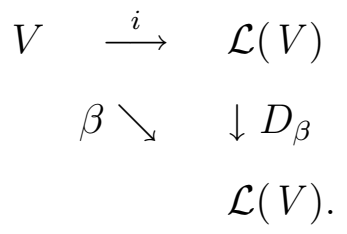

The category of $D G$-Lie algebras and their homomorphisms is denoted by $D L A$.

In the category of $D G$-Lie algebras there exists coproducts [8]. The coproduct of $L$ and $M$ is denoted by $L \coprod M$. If $\mathcal{L} V$ is a free Lie algebra over vector space $V$, then $\mathcal{L}(V) \amalg \mathcal{L}(V)=\mathcal{L}(V \oplus V)$.

Let $(L, d, \varphi)$ be a DG-Lie algebra. A DG-Lie algebra $\left(L^{*}, d^{*}, \varphi^{*}\right)$ together with homomorphisms

$$
(L, d, \varphi) \underset{j_{2}}{\stackrel{j_{1}}{\longrightarrow}}\left(L^{*}, d^{*}, \varphi^{*}\right) \stackrel{p}{\rightarrow}(L, d, \varphi)
$$

is called a cylinder on $(L, d, \varphi)$ if

(i) $j_{1} \amalg j_{2}:(L, d, \varphi) \amalg(L, d, \varphi) \rightarrow\left(L^{*}, d^{*}, \varphi^{*}\right)$ is injective;

(ii) $p j_{1}=p j_{2}=1$; and

(iii) $p$ is a quasi-isomorphism.

Two DGA homomorphisms $f, g:\left(L, d_{L}, \varphi_{L}\right) \rightarrow\left(M, d_{M}, \varphi_{M}\right)$ are called homotopic, $f \sim g$, if $f \amalg g:\left(L, d_{L}, \varphi_{L}\right) \amalg\left(L, d_{L}, \varphi_{L}\right) \rightarrow\left(M, d_{M}, \varphi_{M}\right)$ extends over some cylinder $\left(L^{*}, d^{*}, \varphi^{*}\right)$, see $[3]$. 


\subsection{LIE COALGEBRA}

This subsection is completely dual to the previous one; for more details see [1], [25], $[35]$.

Definition 6. A Lie coalgebra over $R$ is a pair $(M, \psi)$, where $M$ is a vector space and $\psi: M \rightarrow M \otimes M$ is a linear map subject to the conditions

$$
\begin{aligned}
& \text { (1) } \operatorname{Im} \psi \subset \operatorname{Im}(1-T) \\
& \text { (2) }\left(1+\xi+\xi^{2}\right)(1 \otimes \psi) \psi=0
\end{aligned}
$$

A graded Lie coalgebra $M$ is called $t$-reduced if $M_{n}=0$ for $n<t$; a 1-reduced Lie coalgebra is also called connected.

Definition 7. A subspace $W$ is called a Lie subcoalgebra of a Lie coalgebra $M$ if $\psi(W) \subset W \otimes W$.

We give here two familiar examples of a Lie coalgebra which we shall use in further definitions:

Example (1): Let $(C, \Delta)$ be a connected coassociative coalgebra, then $(J C, J \psi-$ $T J \psi)$ is a Lie coalgebra denoted by $l^{c}(C)$. Recall that by $J C$ we denoted a coaugmentation ideal of coalgebra $C$, Subsection 1.3.

Example (2): Let $(H, \mu, \Delta)$ be a connected Hopf algebra with coassociative diagonal. The $Q(H)$, the space of indecomposables of $H$, carries a structure of a Lie coalgebra. Recall that $Q(H)$ can be considered to be a quotient space

$$
Q(I H)=\frac{I H}{I m(I \mu)}=\frac{I H}{I H \cdot I H} .
$$

Since the multiplication $\mu$ of $(H, \mu, \Delta)$ is a coalgebra map, we easily check that $(Q H, I \Delta-T I \Delta)$ is a Lie coalgebra.

Definition 8. A homomorphism $f:\left(M_{1}, \psi_{1}\right) \rightarrow\left(M_{2}, \psi_{2}\right)$ is called a Lie coalgebra homomorphism if $\psi_{2} f=(f \otimes f) \varphi_{1}$. 
The category of Lie coalgebras and Lie coalgebra homomorphisms is denoted by $L C$.

Let $V$ be a vector space with $V_{0}=0$, and $\left(T^{c} V, \Delta, \mu_{s h}\right)$ be a connected Hopf algebra over $V$.

Definition 9. The free Lie coalgebra over vector space $V$ is defined as a quotient space $Q\left(T V, \Delta, \mu_{s h}\right)$.

The free Lie coalgebra over vector space $V$ is denoted by $\mathcal{L}^{c}(V)$.

From Subsection 1.7 we know that any graded coalgebra map $f: C \rightarrow T^{c}(V)$, where $C$ is a connected graded coalgebra, is uniquely defined by the projection

$$
\alpha=P_{1} f J: J C \rightarrow C \rightarrow T^{c}(V) \rightarrow V
$$

and $f=f_{\alpha}$. Let $(C, \psi, \mu)$ be any connected commutative graded Hopf algebra and

$$
f_{\alpha}:(C, \psi) \rightarrow\left(T^{c}(V), \Delta\right)
$$

any graded coalgebra homomorphism. Then $f_{\alpha}:(C, \psi, \mu) \rightarrow\left(T^{c}(V), \Delta, \mu_{s h}\right)$ is a graded Hopf algebra homomorphism if and only if there exists a homomorphism $\alpha^{\prime}: Q C \rightarrow V$ such that $\alpha=\alpha^{\prime} q$, i.e. the following diagram commutes

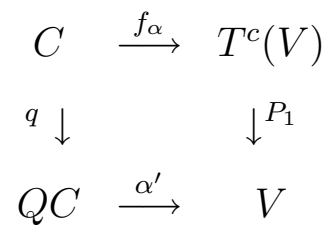

where $q: C \rightarrow Q C=\frac{C}{C \cdot C}$ is a quotient map. Note that commutativity of the diagram implies that $\alpha$ vanishes on $C \cdot C$.

We also know that any coderivation $D: T^{c}(V) \rightarrow T^{c}(V)$ is uniquely defined by the projection

$$
\beta=P_{1} D J: J T^{c}(V) \rightarrow T^{c}(V) \rightarrow T^{c}(V) \rightarrow V
$$

and $D=D_{\beta}$. Let

$$
D_{\beta}:\left(T^{c}(V), \Delta\right) \rightarrow\left(T^{c}(V), \Delta\right)
$$


be any coderivation. Then $D_{\beta}$ is also derivation with respect to $\mu_{s h}$ (i.e. $D_{\beta}$ is a Hopf derivation) if and only if there exists a homomorphism $\beta^{\prime}: Q T^{c}(V)=\mathcal{L}^{c}(V) \rightarrow V$ such that $\beta=\beta^{\prime} q$, i.e. the following diagram commutes

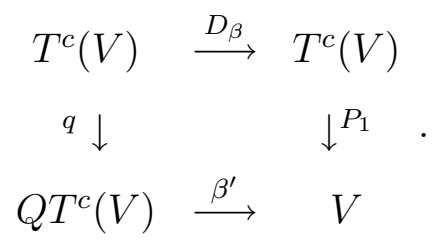

Note that commutativity of the diagram implies that $\beta$ vanishes on the shuffles.

The free Lie coalgebra $\mathcal{L}^{c}(V)$ has the analogous universal property as tensor coalgebra $T^{c}(V)$ : if $M$ is any connected graded Lie coalgebra and $\alpha: M \rightarrow V$ is any homomorphism, there is a unique Lie coalgebra map $f_{\alpha}: M \rightarrow \mathcal{L}^{c}(V)$ which makes the following diagram commute:

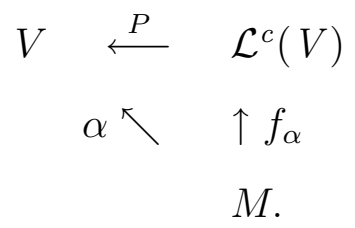

Definition 10. The universal coenveloping coalgebra $U^{c} M$ of a Lie coalgebra $(M, \psi)$ is an object in DGC together with a morphism

$$
\pi_{U^{c} M}: l^{c}\left(U^{c} M\right) \rightarrow M
$$

in $L C$, such that for any $C \in D G C$ and any morphism $f: l^{c}(C) \rightarrow M$ in $L C$, there exists a unique morphism $F: C \rightarrow U^{c} M$ of DGC making the diagram

$$
\begin{array}{cc}
M \stackrel{\pi_{U^{c} M}}{\longleftarrow} & l^{c}\left(U^{c} M\right) \\
f \nwarrow & \uparrow l^{c}(F) \\
& l^{c}(C)
\end{array}
$$

commutative.

Let us look at the construction of $U^{c} M$; Let $\left(T^{c} M, \Delta\right)$ be a tensor coalgebra. The universal coenveloping coalgebra $U^{c} M$ is the largest subcoalgebra of $\left(T^{c} M, \Delta\right)$ 
contained in

$$
\operatorname{ker}\left\{\left(\pi_{T^{c} M} \otimes \pi_{T^{c} M}\right)(1-T) \Delta-\psi \pi_{T^{c} M}\right\}
$$

where $\pi_{T^{c} M}: T^{c} M \rightarrow M$ is the natural projection.

In fact, $U^{c} M$ inherits from $\left(T^{c} M, \Delta, \mu_{s h}\right)$ an algebra structure too. We mention once and for all that $\mu_{U^{c} M}$ makes $U^{c} M$ into a commutative associative Hopf algebra; moreover, there is a natural Hopf algebra isomorphism

$$
U^{c} \mathcal{L}^{c}(M)=T^{c} M
$$

A differential graded Lie coalgebra is a connected graded Lie coalgebra $(M, \psi)$ together with a coderivation $\delta$, i.e. $\psi \delta=(\delta \otimes 1-1 \otimes \delta) \psi$, of degree 1 whose square is 0 .

A differential graded Lie coalgebra homomorphism

$$
f:\left(M_{1}, \psi_{1}, \delta_{1}\right) \rightarrow\left(M_{2}, \psi_{2}, \delta_{2}\right)
$$

is a graded Lie coalgebra homomorphism $f:\left(M_{1}, \psi_{1}\right) \rightarrow\left(M_{2}, \psi_{2}\right)$ with $f \delta=\delta f$.

The free Lie coalgebra $\mathcal{L}^{c}(V)$ has the analogous universal property with respect to coderivations: for any homomorphism $\beta: \mathcal{L}^{c}(V) \rightarrow V$ there is a unique coderivation $D_{\beta}: \mathcal{L}^{c}(V) \rightarrow \mathcal{L}^{c}(V)$ which makes the following diagram commute:

$$
\begin{aligned}
V \quad \stackrel{P}{\longleftarrow} & \mathcal{L}^{c}(V) \\
\beta \nwarrow & \uparrow D_{\beta} \\
& \mathcal{L}^{c}(V) .
\end{aligned}
$$

The category of $D G$-Lie coalgebras and their homomorphisms is denoted by $D L C$. In the category of $D G$-Lie coalgebras there exist products. The product of $M$ and $N$ is denoted by $M \prod N$. The universal properties imply that $\mathcal{L}^{c}(V) \prod \mathcal{L}^{c}(V)=$ $\mathcal{L}^{c}(V \oplus V)$. 
Let $(N, d, \psi)$ be a $D G$-Lie coalgebra. A $D G$-Lie coalgebra $\left(N^{*}, d^{*}, \psi^{*}\right)$ together with homomorphisms

$$
(N, d, \psi) \stackrel{i}{\longrightarrow}\left(N^{*}, d^{*}, \psi^{*}\right) \underset{p_{2}}{\stackrel{p_{1}}{\longrightarrow}}(N, d, \psi)
$$

is called a cylinder on $(N, d, \psi)$ if

(i) $p_{1} \prod p_{2}:\left(N^{*}, d^{*}, \psi^{*}\right) \rightarrow(N, d, \psi) \prod(N, d, \psi)$ is surjective;

(ii) $p_{1} i=p_{2} i=1$; and

(iii) $i$ is a quasi-isomorphism.

Two $D G$-Lie coalgebra homomorphisms $f, g:\left(M, d_{M}, \psi_{M}\right) \rightarrow\left(N, d_{N}, \psi_{N}\right)$ are called homotopic, $f \sim g$, if $f \prod g:\left(M, d_{M}, \psi_{M}\right) \rightarrow\left(N, d_{N}, \psi_{N}\right) \prod\left(N, d_{N}, \psi_{N}\right)$ factors through some cylinder $\left(N^{*}, d^{*}, \psi^{*}\right)$.

\section{$1.10 A_{\infty}$-ALGEBRA}

We shall use the following notion of $A_{\infty}$-algebra (strongly homotopy associative) introduced by Stasheff in [41].

Definition 11. An $A_{\infty}$-algebra $\left(A,\left\{m_{i}\right\}\right)$ is defined as an $R$-Module $A$ together with a set operations $\left\{m_{i}: A^{\otimes i} \rightarrow A \mid \operatorname{deg}\left(m_{i}\right)=2-i\right\}_{i \geq 1}$ satisfying the following relations:

$$
\sum_{k=0}^{n-1} \sum_{j=1}^{n-k}(-1)^{k} m_{n-j+1}\left(\hat{a}_{1}, \cdots, \hat{a}_{k}, m_{j}\left(a_{k+1}, \cdots, a_{k+j}\right), \cdots, a_{n}\right)=0
$$

where $\hat{a}_{i}=(-1)^{\left|a_{i}\right|} a_{i}$.

There exists an alternative but equivalent set of signs, see [23], [31], etc.

An $A_{\infty}$-algebra $\left(A,\left\{m_{i}\right\}\right)$ is called minimal if $m_{1}=0$ [24], [25].

The DGA $(A, d, \mu)$ can be considered as an $A_{\infty}$-algebra of the following type $\left(A,\left\{m_{1}, m_{2}, 0,0, \cdots\right\}\right)$, where $m_{1}=d$, and $m_{2}(a \otimes b)=(-1)^{|a|} \mu(a \otimes b)$. 
The Bar construction on an $A_{\infty}$-algebra $\left(A,\left\{m_{i}\right\}\right)$ is the DGC $B A=T^{c}(s I A)$ with differential

$$
\begin{aligned}
& d_{m}\left[a_{1}|\cdots| a_{n}\right]= \\
& \quad \sum_{i=0}^{n-k} \sum_{k=1}^{n}(-1)^{k+\epsilon_{i}(k)}\left[a_{1}|\cdots| a_{i}\left|m_{k}\left(a_{i+1} \cdots a_{i+k}\right)\right| a_{i+k+1}|\cdots| a_{n}\right],
\end{aligned}
$$

where

$$
\epsilon_{i}(k)=\sum_{t=1}^{i}\left(\left|a_{t}\right|+1\right)+\sum_{j=1}^{k-1}\left(\left|a_{i+j}\right|+1\right)(k-j) .
$$

Conversely, any differential $d$ on the tensor coalgebra $\left(T^{c}(s I A), \Delta\right)$ on a graded module $s I M$ uniquely defines an $A_{\infty}$-algebra structure on $\mathrm{M}$. The operations $m_{j}$ are given by the composition

$$
m_{j}: M^{\otimes j} \stackrel{s^{\otimes j}}{\rightarrow}(s M)^{\otimes j} \stackrel{i}{\rightarrow} T^{c}(s I A) \stackrel{d}{\rightarrow} T^{c}(s I A) \stackrel{P_{1}}{\rightarrow} s M \stackrel{s^{-1}}{\rightarrow} M
$$

The condition $d^{2}=0$ guarantees that such defined $\left\{m_{i}\right\}$ satisfy $(2)$.

Definition 12. Given $A_{\infty}$-algebras $\left(M,\left\{m_{i}\right\}\right)$ and $\left(N,\left\{m_{j}^{\prime}\right\}\right)$, an $A_{\infty}$-algebra morphism $\left(M,\left\{m_{i}\right\}\right) \rightarrow\left(N,\left\{m_{j}^{\prime}\right\}\right)$ is a collection of maps

$$
\left\{f_{i}: M^{\otimes i} \rightarrow N, \operatorname{deg}\left(f_{i}\right)=i-1\right\}_{i \geq 1}
$$

satisfying the following identities

$$
\begin{aligned}
& \sum_{k=0}^{n-1} \sum_{j=1}^{n-k}(-1)^{k} f_{n-j+1}\left(\hat{a}_{1}, \cdots, \hat{a}_{k}, m_{j}\left(a_{k+1}, \cdots, a_{k+j}\right), \cdots, a_{n}\right)= \\
& =\sum_{t=1}^{n} \sum_{k_{1}+\cdots+k_{t}=n} m_{t}^{\prime}\left(f_{k_{1}}\left(a_{1}, \cdots, a_{k_{1}}\right), \cdots, f_{k_{t}}\left(a_{n-k_{t}+1}, \cdots, a_{n}\right)\right) .
\end{aligned}
$$

It is clear from the definition that the first component $f_{1}:\left(M, m_{1}\right) \rightarrow\left(N, m_{1}^{\prime}\right)$ is a chain map.

An $A_{\infty}$-algebra morphism $\left\{f_{i}\right\}:\left(M,\left\{m_{i}\right\}\right) \rightarrow\left(N,\left\{m_{i}^{\prime}\right\}\right)$ is called an isomorphism (resp. quasi-isomorphism) if the first component $f_{1}:\left(M, m_{1}\right) \rightarrow\left(N, m_{1}^{\prime}\right)$ is an isomorphism (resp. quasi-isomorphism). 
Definition 13. A morphism of $A_{\infty}$-algebras $\left\{f_{i}\right\}:\left(M,\left\{m_{i}\right\}\right) \rightarrow\left(N,\left\{\bar{m}_{j}\right\}\right)$ induces a DGC map of Bar constructions $B\left\{f_{i}\right\}: B M \rightarrow B N$ given by

$$
B\left\{f_{i}\right\}=\sum_{i_{1}+\cdots+i_{k}=n} \sum_{k=1}^{n}\left[f_{i_{1}}\left(s^{-1}\right)^{\otimes i_{1}}|\cdots| f_{i_{k}}\left(s^{-1}\right)^{\otimes i_{k}}\right] .
$$

where $s^{-1}$ is a suspension. It is easy to prove that $B\left\{f_{i}\right\}$ is an isomorphism (resp. quasi-isomorphism) if and only if $f_{1}: M \rightarrow N$ is an isomorphism (resp. quasiisomorphism) (see [25], for example).

Conversely, any DG-coalgebra map $f:\left(B M, d_{M}, \Delta\right) \rightarrow\left(B N, d_{N}, \Delta\right)$ uniquely defines $A_{\infty}$-algebra morphisms. The operations $f_{j}$ are given by the composition

$$
P_{1} f=\left\{f_{j}: M^{\otimes j} \stackrel{s^{\otimes j}}{\rightarrow}(s M)^{\otimes j} \stackrel{i}{\rightarrow} B M \stackrel{f}{\rightarrow} B N \stackrel{P_{1}}{\rightarrow} s N \stackrel{s^{-1}}{\rightarrow} N\right\}
$$

$A_{\infty}$-algebras and $A_{\infty}$-algebra morphisms form the $A_{\infty}$-algebra category, which we denote by $A_{\infty}-\mathrm{Alg}$.

There is one more category to consider- category DASH (see [37], [42]); it can be considered as a subcategory of $A_{\infty}$-Alg. Objects in DASH are $A_{\infty}$-algebras of type $\left(A,\left\{m_{1}, m_{2}, 0,0, \cdots\right\}\right)$, i.e. DGA, and morphisms-ordinary morphisms in $A_{\infty}$-Alg.

Definition 14. An $A_{\infty}$-algebra $\left(M,\left\{m_{i}\right\}\right)$ is called commutative if

$$
m_{n} s_{i, n-i}=0
$$

for $1 \leq i \leq n-1, n \geq 2$, and $s_{i, n-i} \in S_{i, n-i} ;$ see $[\mathbf{2 4}],[\mathbf{2 5}]$.

Some authors also call such $A_{\infty}$-algebra a balanced $A_{\infty}$-algebra [33].

The Bar-construction of a commutative $A_{\infty}$-algebra $\left(A,\left\{m_{i}\right\}\right)$ is a DG-Hopf algebra with respect to shuffle products, i.e. $\left(B A, d_{A}, \Delta, \mu_{s h}\right) \in H A$. And conversely, any differential on the DG-Hopf algebra $\left(B A, d_{A}, \Delta, \mu_{s h}\right)$ produces commutative $A_{\infty}$ algebra on $A$.

Definition 15. Given two commutative $A_{\infty}$-algebras $\left(M,\left\{m_{i}\right\}\right)$ and $\left(N,\left\{\bar{m}_{i}\right\}\right)$. An $A_{\infty}$-algebra morphism $\left\{f_{i}\right\}:\left(M,\left\{m_{i}\right\}\right) \rightarrow\left(N,\left\{\bar{m}_{j}\right\}\right)$ is called a commutative 
$A_{\infty}$-algebra morphism if

$$
f_{n} s_{i, n-i}=0
$$

for $1 \leq i \leq n-1, n \geq 2$, and $s_{i, n-i} \in S_{i, n-i}$; see $[\mathbf{2 4}],[\mathbf{2 5}]$.

As we have mentioned above, any $A_{\infty}$-algebra morphism

$$
\left\{f_{i}\right\}:\left(M,\left\{m_{i}\right\}\right) \rightarrow\left(N,\left\{\bar{m}_{j}\right\}\right)
$$

induces a DG-coalgebra map of appropriate bar constructions $B\{f\}:\left(B M, d_{M}, \Delta\right) \rightarrow$ $\left(B N, d_{N}, \Delta\right)$. If $\left\{f_{i}\right\}:\left(M,\left\{m_{i}\right\}\right) \rightarrow\left(N,\left\{\bar{m}_{j}\right\}\right)$ is a commutative $A_{\infty}$-algebra morphism then

$$
B\{f\}:\left(B M, d_{M}, \Delta, \mu_{s h}\right) \rightarrow\left(B N, d_{N}, \Delta, \mu_{s h}\right)
$$

is a DG-Hopf algebra map. Conversely, any DG-Hopf algebra map

$$
f:\left(B M, d_{M}, \Delta, \mu_{s h}\right) \rightarrow\left(B N, d_{N}, \Delta, \mu_{s h}\right)
$$

produces a commutative $A_{\infty}$-algebra morphism

$$
f_{i}: M^{\otimes i} \rightarrow(s M)^{\otimes i} \rightarrow B M \rightarrow B N \rightarrow s N \rightarrow N
$$

Commutative $A_{\infty}$-algebras and their morphisms form a commutative $A_{\infty}$-algebra category, which is denoted by $C-A_{\infty}-\mathrm{Alg}$.

\subsection{Twisting COCHAin}

In this section we recall briefly the definition of twisting cochains of Brown [11] and some notions and known facts related with them.

Definition 16. Let $\left(C, d_{C}, \triangle, \varepsilon_{C}, \eta_{C}\right)$ be a $D G C$ and $\left(A, d_{A}, \mu, \varepsilon_{A}, \eta_{A}\right)$ a DGA. The complex $\operatorname{Hom}(C, A)$ is defined as a DGA with differential

$$
D f=d \cdot f-(-1)^{|f|} f \cdot d_{C}
$$


and multiplication

$$
f \cdot g=\mu(f \otimes g) \triangle, \quad f, g \in \operatorname{Hom}(C, A) .
$$

The neutral element in $\operatorname{Hom}(C, A)$ is a composition $e: C \stackrel{\varepsilon_{C}}{\rightarrow} R \stackrel{\eta_{A}}{\rightarrow} A$.

It is easy to check that

$$
D(f \cdot g)=D f \cdot g+(-1)^{|f|} f \cdot D g .
$$

From here throughout the paper we shall work only with augmentation and coaugmentation ideals of DGA and DGC. Thus we consider the complex $\operatorname{Hom}(J C, I A)$.

Definition 17. The element $t \in \operatorname{Hom}(J C, I A)$ of degree -1 is a twisting cochain in the sense of Brown [11] if it satisfies

$$
D t=-t \cdot t
$$

It is easy to show that for any $D G A\left(A, d_{A}, \mu\right)$ and for any $D G C\left(C, d_{C}, \triangle\right)$ the natural maps

$$
P_{1}: B A \rightarrow A \quad \text { and } i_{1}: C \rightarrow \Omega C
$$

are twisting cochains, see [37].

Let $\alpha: J C \rightarrow I A$ be a map of degree -1. By the universal properties mentioned in Subsections 1.6 and 1.7, any such homomorphism uniquely defines an algebra map $f_{\alpha}: \Omega C \rightarrow A$ and a coalgebra map $g_{\alpha}: C \rightarrow B A$. Homomorphisms $f_{\alpha}: \Omega C \rightarrow A$ and $g_{\alpha}: C \rightarrow B A$ are chain maps, i.e. maps in $D G A$ and $D G C$ respectively, if and only if $\alpha: J C \rightarrow I A$ is a twisting cochain; see [25], for example. Thus there are bijections

$$
H_{D \rightarrow}(C, B A) \leftrightarrow T(J C, I A) \leftrightarrow \operatorname{Hom}_{D G A}(\Omega C, A)
$$

where by $T(J C, I A)$ we denote the set of all twisting cochains from $J C$ to $I A$.

Berikashvili in [9] introduced the following equivalence relation on $T(J C, I A)$. 
Definition 18. Two twisting cochains $\alpha, \alpha^{\prime}: J C \rightarrow I A$ are equivalent (we write $\alpha \sim \alpha^{\prime}$ ) if there exists a homomorphism

$$
\beta: J C \rightarrow I A
$$

of degree 0 satisfying the following identity

$$
\alpha-\alpha^{\prime}=\beta d_{C}-d_{A} \beta+\beta \cdot \alpha^{\prime}-\alpha \cdot \beta .
$$

The set $T(C, A)$ factored by this equivalence relation is denoted by $D(C, A)$.

Let $f: A \rightarrow B$ be a map in $D G A$. It is trivial to check that for any $\alpha \in T(J C, I A)$ the composition If $\alpha$, where If $I A \rightarrow I B$, is a twisting cochain in $T(J C, I B)$. Moreover, if $\alpha \sim \alpha^{\prime}$, then If $\alpha \sim \operatorname{If} \alpha^{\prime}$. Thus for any DGC C, a DGA map $f: A \rightarrow B$ induces a set map

$$
f_{*}: D(J C, I A) \rightarrow D(J C, I B)
$$

defined by $[\alpha] \longmapsto[I f \alpha]$. Moreover Berikashvili in $[\mathbf{1 0}]$ proved the following

Theorem 19. Let $f: A \rightarrow B$ be a map in DGA inducing an isomorphism in homology. Then

$$
f_{*}: D(J C, I A) \rightarrow D(J C, I B)
$$

is a bijection.

Let $f=\left\{f_{i}\right\}: A \rightarrow B$ be a DASH map. Kadeishvili in [26] showed that if $\alpha \in T(J C, I A)$, then

$$
\phi=\sum_{i} J f_{i}(\alpha \otimes \cdots \otimes \alpha) \triangle^{i}
$$

belongs to $T(J C, I B)$. Thus for any DGC $C$, a DASH map $f=\left\{f_{i}\right\}: A \rightarrow B$ induces a set map

$$
T(f): T(J C, I A) \rightarrow T(J C, I B)
$$

$\left(\alpha \longmapsto \sum_{i} J f_{i}(\alpha \otimes \cdots \otimes \alpha) J \triangle^{i}\right)$, which by itself induces a set map

$$
f_{*}: D T(J C, I A) \rightarrow D(J C, I B)
$$


defined by $[\alpha] \longmapsto\left[\sum_{i} J f_{i}(\alpha \otimes \cdots \otimes \alpha) J \triangle^{i}\right]$. Kadeishvili in [26] generalized Theorem 19 in the following way

Theorem 20. Let $f=\left\{f_{i}\right\}: A \rightarrow B$ be a map in DASH, such that its first component $f_{1}: A \rightarrow B$ induces an isomorphism in homology. Then

$$
f_{*}: D(J C, I A) \rightarrow D T(J C, I B)
$$

is a bijection.

It is known that if $\alpha, \alpha^{\prime}: J C \rightarrow I A$ are equivalent twisting cochains, then $f_{\alpha}, f_{\alpha^{\prime}}$ : $\Omega C \rightarrow A\left(\right.$ resp. $\left.g_{\alpha}, g_{\alpha^{\prime}}: C \rightarrow B A\right)$ are homotopic in $D G A$ (resp. $D G C$ ); see [27], for example.

We complete this section with the following

THEOREM 21 ([30]). Let $K$ be a simplicial set.

(a) Then in the DGA $A=\operatorname{Hom}\left(C_{*}(K), \Omega C_{*}(K) \otimes \Omega C_{*}(K)\right)$ there exists the element E of degree -1, satisfying the following conditions

I) $D E=-E \cdot E$ (i.e. E is a twisting cochain)

II) The components $E^{1,0}: C \rightarrow C \otimes R$ and $E^{0,1}: C \rightarrow R \otimes C$ are given by

$$
\begin{aligned}
& E^{1,0}(x)=-x \otimes 1 \\
& E^{0,1}(x)=-1 \otimes x
\end{aligned}
$$

III) $E^{n, 0}=E^{0, n}=0$ when $n \neq 1$.

(b) Let $E$ and $\bar{E}$ be any twisting cochains in $A$ satisfying II) and III); i.e. $E^{1,0}=$ $\bar{E}^{1,0}, E^{0,1}=\bar{E}^{0,1}$, and $E^{n, 0}=\bar{E}^{n, 0}=0, E^{0, n}=\bar{E}^{0, n}=0$. Then there exists $P \in A$ of degree 0 such that $P^{0,0}=0$ and

$$
\bar{E}=E+P \cdot E-\bar{E} \cdot P-D P \text {. }
$$

The notation in the theorem is not very obvious. We clarify it below. Let us denote by $p^{\otimes^{i}}: \Omega C_{*}(K) \rightarrow C_{*}(K)^{\otimes^{i}}, i=0,1,2 \cdots$, the natural projections on the Cobarconstruction. Also notice that any homomorphism $F: C_{*}(K) \rightarrow \Omega C_{*}(K) \otimes \Omega C_{*}(K)$ 
uniquely defines the set of linear maps

$$
\left\{F^{m, n}: C_{*}(K) \stackrel{F}{\rightarrow} \Omega C_{*}(K) \otimes \Omega C_{*}(K) \stackrel{p^{\otimes^{m}} \otimes p^{\otimes^{n}}}{\longrightarrow}\left(C_{*}(K)^{\otimes^{m}}\right) \otimes\left(C_{*}(K)^{\otimes^{n}}\right)\right\}
$$

called the components of homomorphism $F$. Part (a) of the theorem says that on $\operatorname{Hom}\left(C_{*}(K), \Omega C_{*}(K) \otimes \Omega C_{*}(K)\right)$ we have a special twisting cochain $E$ (with the components from $I I$ and $I I I)$; part (b) asserts that this twisting cochain is defined uniquely up to twisting equivalence in sense of Definition 18.

\subsection{Perturbation Lemma}

We start this section with the description of the category $S D R$. It is defined as follows: an object in $S D R$ is a set

$$
\tau=\left(\left(X, d_{X}\right) \underset{\beta}{\stackrel{\alpha}{\rightleftarrows}}\left(\left(Y, d_{Y}\right) ; \nu\right)\right)
$$

where $(1)\left(X, d_{X}\right)$ and $\left(Y, d_{Y}\right)$ are $D G$-vector spaces with increasing filtration

$$
\subset F_{p} X \subset F_{p+1} X \subset \cdots, \quad \subset F_{p} Y \subset F_{p+1} Y \subset \cdots
$$

(2) $\beta$ and $\alpha$ are morphisms of DG-vector spaces preserving filtration and satisfying

$$
\beta \alpha=1_{X}, \quad \alpha \beta \stackrel{\nu}{\sim} 1_{Y} \text {, i.e. } 1_{Y}-\alpha \beta=d_{Y} \nu+\nu d_{Y}
$$

$$
\nu \alpha=0, \quad \beta \nu=0, \quad \nu \nu=0 .
$$

The morphism in $S D R$

$$
(f, g): \tau=\left(\left(X, d_{X}\right) \underset{\beta}{\stackrel{\alpha}{\rightleftarrows}}\left(\left(Y, d_{Y}\right) ; \nu\right)\right) \rightarrow \tau \prime=\left(\left(X^{\prime}, d_{X}^{\prime}\right) \underset{\beta^{\prime}}{\stackrel{\alpha^{\prime}}{\rightleftarrows}}\left(\left(Y^{\prime}, d_{Y}^{\prime}\right) ; \nu^{\prime}\right)\right)
$$

is defined as a pair of chain maps $f: X \rightarrow X^{\prime}$ and $g: Y \rightarrow Y^{\prime}$ preserving filtration and satisfying

$$
g \alpha=\alpha^{\prime} f, \quad f \beta=\beta^{\prime} g, \quad g \nu=\nu^{\prime} g .
$$


Let $(C, d)$ be a chain complex over the field. There is a familiar $S D R$, more usually known as $S D R$ data, associated with this chain complex

$$
\tau=((H(C), d=0) \underset{g}{\stackrel{f}{\rightleftarrows}}((C, d) ; \nu) .
$$

Let us discuss this $S D R$ more closely. We have 2 splitting short exact sequences

$$
0 \rightarrow B_{n} \stackrel{j_{n}}{\longrightarrow} Z_{n} \stackrel{\stackrel{c}{\rightleftarrows}}{\stackrel{p_{1}}{\rightleftarrows}} H_{n} \rightarrow 0
$$

and

$$
0 \rightarrow Z_{n} \stackrel{i_{n}}{\rightarrow} C_{n} \underset{p_{2}}{\stackrel{d_{n}}{\rightleftarrows}} B_{n-1} \rightarrow 0
$$

where $p_{1}$ and $p_{2}$ are splitting maps (of course not unique), and $c l$ is a homomorphism assigning to each n-cycle $z$ its homology class $c l(z)$. Then we have two isomorphisms $Z_{n} \cong B_{n} \oplus H_{n}$ and $C_{n} \cong Z_{n} \oplus B_{n-1}$. They yield to an isomorphism

$$
\gamma_{n}: C_{n} \cong B_{n} \oplus H_{n} \oplus B_{n-1}
$$

given by $\gamma\left(c_{n}\right)=\left(\left(c_{n}-p_{2} d_{n} c_{n}\right)-p_{1} c l\left(c_{n}-p_{2} d_{n} c_{n}\right), c l\left(c_{n}-p_{2} d_{n} c_{n}\right), d_{n} c_{n}\right)$, [40]. Let us define a differential $\delta$ on $B_{n} \oplus H_{n} \oplus B_{n-1}$ by

$$
\delta\left(b_{n}, h_{n}, b_{n-1}\right)=\left(b_{n-1}, 0,0\right)
$$

We see that $\delta$ is independent of the choice of the splittings maps, it depends only on d. Clearly, $\delta^{2}=0$ and $\operatorname{ker}\left(\delta_{n}\right) / \operatorname{im}\left(\delta_{n+1}\right)=H_{n}$. It is straightforward to check that $\gamma=\left\{\gamma_{n}\right\}$ is a chain complex isomorphism, i.e. $\gamma_{n-1} \delta_{n}=d_{n} \gamma_{n}$. Once the splitting maps are fixed we identify the chain complexes $\left(C_{n}, d_{n}\right)$ and $\left(B_{n} \oplus H_{n} \oplus B_{n-1}, \delta_{n}\right)$ through $\gamma$.

We define the maps $f: H_{n} \rightarrow C_{n}, g: C_{n} \rightarrow H_{n}$, and $\nu: C_{n} \rightarrow C_{n+1}$ by the following formulas

$$
f\left(h_{n}\right)=\left(0, h_{n}, 0\right), \quad g\left(b_{n}, h_{n}, b_{n-1}\right)=h_{n}, \quad \nu\left(b_{n}, h_{n}, b_{n-1}\right)=\left(0,0,-b_{n}\right) .
$$

It is immediate from the definitions that $g f=1, f g-1=\nu \delta+\delta \nu, \nu f=0$, $g \nu=0, \nu \nu=0$. 
Definition 22. Let $\left(Y, d_{Y}\right)$ be a $D G$-vector space. A perturbation of the differential $d_{Y}$ is a homomorphism $t: Y \rightarrow Y$ of degree 1 satisfying $d_{Y} t+t d_{Y}+t t=0$ (this condition guarantees that $\left(d_{Y}+t\right)^{2}=0$, i.e. $d_{Y}+t$ is a new differential) and $t\left(F_{p} Y\right) \subset F_{p-1} Y$ (decreases filtration).

Let us denote by $S D R P$ the category of objects $(\tau, t)$, where

$$
\tau=\left(\left(X, d_{X}\right) \underset{\beta}{\stackrel{\alpha}{\rightleftarrows}}\left(\left(Y, d_{Y}\right) ; \nu\right)\right)
$$

is an object in $S D R$, and $t$ is a perturbation on $Y$. A morphism in $\operatorname{SDRP}(\tau, t) \rightarrow$ $\left(\tau^{\prime}, t^{\prime}\right)$ is a $S D R$ morphism $(f, g):\left(\tau \rightarrow \tau^{\prime}\right)$ satisfying $g t=t^{\prime} g$. We are ready now to state the basic perturbation lemma [12]

LEMMA 23. Let

$$
\tau=\left(\left(X, d_{X}\right) \underset{\beta}{\stackrel{\alpha}{\rightleftarrows}}\left(\left(Y, d_{Y}\right) ; \nu\right)\right)
$$

be an $S D R$ and $t: Y \rightarrow Y$ any perturbation on $Y$. Then the formulas

$$
\begin{aligned}
& t^{\infty}=\beta t \alpha-\beta t \nu t \alpha+\beta t \nu t \nu t \alpha-\cdots \\
& \alpha^{\infty}=\alpha-\nu t \alpha+\nu t \nu t \alpha-\cdots \\
& \beta^{\infty}=\beta-\beta t \nu+\beta t \nu t \nu-\cdots \\
& \nu^{\infty}=\nu-\nu t \nu+\nu t \nu t \nu-\cdots
\end{aligned}
$$

define new (perturbed) SDR

$$
\tau^{\infty}=\left(\left(X, d_{X}+t^{\infty}\right) \underset{\beta^{\infty}}{\stackrel{\alpha^{\infty}}{\rightleftarrows}}\left(\left(Y, d_{Y}+t\right) ; \nu^{\infty}\right)\right) ;
$$

The construction of $\tau^{\infty}$ is natural; i.e. the correspondence $(\tau, t) \rightarrow \tau^{\infty}$ defines a functor $F: S D R P \rightarrow S D R$.

Note that we have stated the perturbation lemma above for a $D G$-vector space.

There is an analogous (co)algebra perturbation lemma for $D G$-(co)algebras over the field; in that case if $\tau$ is (co)algebraic, i.e.,

(1) $\left(X, d_{X}\right)$ and $\left(Y, d_{Y}\right)$ are $D G$-(co)algebras; 
(2) $\alpha$ and $\beta$ are $D G$-(co)algebra maps;

(3) $\nu$ is a (co)derivation;

and $t: Y \rightarrow Y$ is (co)derivation, then the resulting $\tau^{\infty}$ is (co)algebraic too [22], [24], $[25]$.

In [23] (see also [39]) was shown that in the homology of 1-connected $D G$-algebra $(A, d, \mu)$ there is an $A_{\infty}$-algebra structure. Later using the machinery of the coalgebra perturbation lemma in [25] (see also [21]) it was shown that in homology of the 1connected commutative $A_{\infty}$-algebra $\left(A,\left\{m_{i}\right\}\right)$ there is a commutative minimal $A_{\infty^{-}}$ algebra structure (over the rationals). We state the theorem below and give a sketch of the proof.

TheOrem 24. Given a 1-connected commutative $A_{\infty}$-algebra $\left(A,\left\{m_{i}\right\}\right)$. Then in homology $H\left(A, m_{1}=d\right)$ there arises a minimal commutative $A_{\infty}$-algebra structure and a commutative $A_{\infty}$-algebra quasi-isomorphism

$$
\left\{f_{i}\right\}:\left(H(A),\left\{m_{i}^{H}\right\}\right) \rightarrow\left(A,\left\{m_{i}\right\}\right) .
$$

Proof. Let $\tau=\left((H(A), d=0) \underset{g}{\stackrel{f}{\rightleftarrows}}\left(\left(A, d_{A}\right) ; \nu\right)\right.$ be $S D R$ data as discussed above. Clearly, the given $S D R$ induces a coalgebra $S D R$

$$
B \tau=((B H(A), d=0) \underset{B g}{\stackrel{B f}{\rightleftarrows}}((B A, B d) ; B \nu) .
$$

( $B$ is a Bar-construction functor).

Let consider the following coderivation (perturbation) on $B A$

$$
t=d_{m}-B d
$$

where $d_{m}$ is a differential of $B A$ defined by an $A_{\infty}$-algebra structure on $\mathrm{A}$ (see definition of differential on Bar construction). Applying the (coalgebra) perturbation lemma to $(B \tau, t)$ we obtain new $S D R$

$$
(B \tau)^{\infty}=\left(\left(B H(A), t^{\infty}\right) \underset{(B g)^{\infty}}{\stackrel{(B f)^{\infty}}{\rightleftarrows}}\left((B A, B d+t) ;(B \nu)^{\infty}\right)\right.
$$


and according to the formulas in the perturbation lemma $t^{\infty}=B(g) t B(f)^{\infty}$.

From Subsection 1.10 we know that any differential $t^{\infty}$ on $B H(A)$ uniquely defines an $A_{\infty}$-algebra structure on $H(A)$. Moreover, since perturbation decreases filtration it was shown in $[\mathbf{2 5}]$ that this is a minimal $A_{\infty}$-algebra structure; i.e. the first component $m_{1}^{H}=0$.

So, it remains to show that operations

$$
m_{j}: H(A) \stackrel{\otimes j}{\stackrel{s^{\otimes j}}{\rightarrow}}(s H(A))^{\otimes j} \stackrel{i}{\rightarrow} B H(A) \stackrel{t^{\infty}}{\rightarrow} B H(A) \stackrel{P_{1}}{\rightarrow} s H(A) \stackrel{s^{-1}}{\rightarrow} H(A)
$$

and

$$
f_{j}: H(A)^{\otimes j} \stackrel{s^{\otimes j}}{\rightarrow}(s H(A))^{\otimes j} \stackrel{i}{\rightarrow} B H(A) \stackrel{(B f)^{\infty}}{\rightarrow} B A \stackrel{P_{1}}{\rightarrow} s A \stackrel{s^{-1}}{\rightarrow} A
$$

vanish on the shuffles. Let us assume that operations $f_{j \geq 2}$ vanish on the shuffles; this implies (Subsection 1.10)

$$
B(f)^{\infty}:\left(B H(A), \mu_{s h}\right) \rightarrow\left(B A, \mu_{s h}\right)
$$

is a multiplicative map. Then

$$
\begin{gathered}
t^{\infty}(a * b)=B(g) t B(f)^{\infty}(a * b)=B(g) t\left[B(f)^{\infty}(a) * B(f)^{\infty}(b)\right]= \\
\left.B(g)\left[t B(f)^{\infty}(a) * B(f)^{\infty}(b)+(-1)^{|a|} B(f)^{\infty}(a) * t B(f)^{\infty}(b)\right)\right]= \\
B(g) t B(f)^{\infty}(a) * B(g) B(f)^{\infty}(b)+(-1)^{|a|} B(g) B(f)^{\infty}(a) * B(g) t B(f)^{\infty}(b)= \\
t^{\infty}(a) * b+(-1)^{|a|} a * t^{\infty}(b) .
\end{gathered}
$$

We have used here that $B(g) B(f)^{\infty}=1$, which easily follows from the definition of $B(f)^{\infty}$ (see formulas in the perturbation lemma) and from the fact that $B(g) B(f)=$ 1. Also we have used multiplicativity of

$$
B(g):\left(B H, \mu_{s h}\right) \rightarrow\left(B A, \mu_{s h}\right)
$$

which easily follows from the fact that $B(g)$ as a $D G$-coalgebra map is generated by the $A_{\infty}$-algebra map

$$
\left\{g_{1}=g, 0,0, \cdots\right\}:\left(H,\left\{m_{i}^{H}\right\}\right) \rightarrow\left(A,\left\{m_{i}\right\}\right)
$$


with a single non-trivial component $g=g_{1}: H \rightarrow A$ and automatically $g_{k} s_{i, k-i}=0$ for $k \geq 2$. This implies that $B(g)$ is multiplicative (Subsection 1.10).

Thus assuming $B(f)^{\infty}$ is multiplicative we obtain $t^{\infty}$ is a derivation of the shuffle product; i.e. $\left(B H, t^{\infty}, \Delta, \mu_{s h}\right) \in H A$. This implies that $t^{\infty}$ produces a commutative $A_{\infty}$-algebra structure on $H$ (Subsection 1.10). So it remains to show that $B(f)^{\infty}$ is multiplicative, or equivalently operations

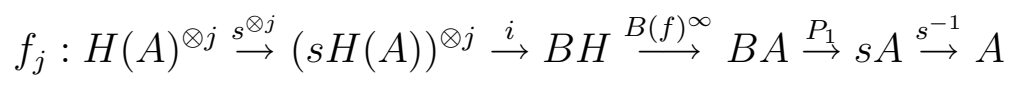

vanish on the shuffles; to check $f_{k} s_{i, k-i}=0$ is straightforward, see [25], where it was shown also that this construction is natural and unique.

REMARK 25. In the proof of Theorem 24 we constructed DG Hopf algebra map

$$
B(f)^{\infty}:\left(B H, t^{\infty}, \Delta, \mu_{s h}\right) \rightarrow\left(B A, d_{m}, \Delta, \mu_{s h}\right)
$$

and its homotopy inverse

$$
B(g)^{\infty}:\left(B A, d_{m}, \Delta, \mu_{s h}\right) \rightarrow\left(B H, t^{\infty}, \Delta, \mu_{s h}\right)
$$

in DGC such that $B(g)^{\infty}$ is DG-coalgebra map with $B(g)^{\infty} B(f)^{\infty}=1_{B H}$, and $B(f)^{\infty} B(g)^{\infty} \sim 1_{B A}$. It is immediate from Lemma 33 that $B(g)^{\infty}$ is multiplicative up to homotopy, i.e. there exists a coderivation homotopy from to $B(g)^{\infty} \mu_{\text {sh }}$ to $\mu_{\text {sh }}\left(B(g)^{\infty} \otimes B(g)^{\infty}\right)$. In the following sections such maps will be called $H A H$ homomorphisms.

\subsection{Hopf ALGEBRAS UP TO HOMOTOPY}

In this section we state several fundamental results of Anick from [3] and [4]. Let $D G A_{0}$ be a category of connected $D G$-algebras over $R$, a commutative ring with unity. Category $D G A_{0}$ has push-outs. The push-out of the diagram $B \stackrel{f}{\leftarrow} A \stackrel{g}{\rightarrow} C$ is denoted by $B \coprod_{A} C$. Most often, we consider push-outs over the trivial graded 
algebra $R$, for which the notation is simply $B \amalg C$. As an R-module, $B \coprod C$ equals the direct sum of the graded $R$-modules

$$
\begin{gathered}
R, B, C, B \otimes C, C \otimes B, C \otimes B \otimes B, B \otimes C \otimes B, B \otimes B \otimes C, \\
B \otimes C \otimes C, C \otimes B \otimes C, C \otimes C \otimes B, \cdots
\end{gathered}
$$

It has an obvious multiplicative structure. If $f$ and $g$ are $D G A$ homomorphisms, then $B \coprod_{A} C$, endowed with the obvious differential, is the push-out in $D G A$ of the di$\operatorname{agram}\left(B, d_{B}, \mu_{B}\right) \stackrel{f}{\leftarrow}\left(A, d_{A}, \mu_{A}\right) \stackrel{g}{\rightarrow}\left(C, d_{C}, \mu_{C}\right)$. There are obvious $D G A$ surjections $A \coprod B \rightarrow A \otimes B \rightarrow A \times B \rightrightarrows A, B$.

Definition 26. Let $(A, d, \mu)$ be a DG-algebra. A DG-algebra $\left(A^{*}, d^{*}, \mu^{*}\right)$ together with homomorphisms

$$
(A, d, \mu) \underset{j_{2}}{\stackrel{j_{1}}{\longrightarrow}}\left(A^{*}, d^{*}, \mu^{*}\right) \stackrel{p}{\longrightarrow}(A, d, \mu)
$$

is called a cylinder on $(A, d, \mu)$ if

(i) $j_{1} \coprod j_{2}:(A, d, \mu) \coprod(A, d, \mu) \rightarrow\left(A^{*}, d^{*}, \mu^{*}\right)$ is injective;

(ii) $p j_{1}=p j_{2}=1$; and

(iii) $p$ is quasi-isomorphism.

Two $D G A$ homomorphisms $f, g:\left(A, d_{A}, \mu_{A}\right) \rightarrow\left(B, d_{B}, \mu_{B}\right)$ are called homotopic, $f \sim g$, if $f \coprod g:\left(A, d, \mu_{A}\right) \coprod\left(A, d, \mu_{A}\right) \rightarrow\left(B, d_{B}, \mu_{B}\right)$ extends over some cylinder $\left(A^{*}, d^{*}, \mu^{*}\right)$.

LEMma 27. (a) if $A \stackrel{h}{\rightarrow} B \underset{g}{\stackrel{f}{\rightarrow}} C \stackrel{i}{\rightarrow} D$ are $D G A$ homomorphisms and $f \sim g$, then ifh $\simeq i g h$.

(b) $\sim$ is an equivalence relation.

Recall that a DG-algebra $\left(A, d, \mu_{A}\right)$ is called free if forgetting the differential it is isomorphic with some $T V$.

In [8], Baues and Lemaire constructed a canonical cylinder on a free DG-algebra A. We denote this Baues-Lemaire cylinder by $I(A, d)$ or $(I A, D)$, and we recall briefly its construction. If $A=T V$, then $I A=T\left(V \oplus V^{\prime} \oplus s V\right)$ as an algebra, where 
$V^{\prime} \cong V$. To define $D$, first put $f, f^{\prime}: T V \rightarrow I A$ by $f(x)=x$ and $f^{\prime}(x)=x^{\prime}$. Let $S$ be the unique $\left(f, f^{\prime}\right)$-derivation having $S(x)=s x$ for $x \in V$. Setting $D(x)=f d(x)$ and $D\left(x^{\prime}\right)=f^{\prime} d(x)$ and $D(s x)=x-x^{\prime}-S d(x)$, extend $D$ (uniquely) to a $(1,1)$ derivation of degree -1 on $I A$. Then $S d+D S$ is an $\left(f, f^{\prime}\right)$-derivation, $f-f^{\prime}=S d+D S . D^{2}=0$ and we have constructed the DG-algebra $(I A, D)$.

Proposition 28. Let $f, g:\left(A, d_{A}, \mu_{A}\right) \rightarrow\left(B, d_{B}, \mu_{B}\right)$ with $A$ free. The following are equivalent

(i) $f \sim g$;

(ii) $f \coprod g:\left(A, d_{A}\right) \coprod\left(A, d_{A}\right) \rightarrow\left(B, d_{B}\right)$ extends over $I(A, d)$

(iii) there is a derivation homotopy from $f$ to $g$.

We have a similar proposition for $D G$-Lie algebras, however, there is no analog for derivation homotopy. The canonical cylinder, due to Tanre [44], is obtained through an entirely new method.

The Tanre cylinder, defined for free DG-Lie algebras over $Q$, is constructed as follows. Given $(\mathcal{L} V, \delta)$, put $I(\mathcal{L} V, \delta)=(I \mathcal{L} V, D)$, where $I \mathcal{L} V=\mathcal{L}(V \oplus s V \oplus D s V)$ and $\left.D\right|_{\mathcal{L} V}=\delta$. The obvious inclusion $j_{1}: \mathcal{L} V \rightarrow I \mathcal{L} V$ is one "end" of the cylinder, and $p: I \mathcal{L} V \rightarrow \mathcal{L} V$ is given on generators by $p(x)=x, p(s x)=0$, and $p(D s x)=0$.

The inclusion $j_{2}$, the other "end" of the cylinder is defined as follows; given a pointwise nilpotent derivation $\theta$ of degree zero on a graded Lie algebra $L$, observe that

$$
e^{\theta}=1+\sum_{n=1}^{\infty} \frac{\theta^{n}}{n !}
$$

defines a Lie algebra automorphism on $L$. Define a derivation $S$ of degree +1 on $I \mathcal{L} V$ by defining it on generators as $S(x)=x, S(s x)=0$, and $S(D s x)=0$. Then $\alpha=[D, S]=D S+S D$ is a derivation on $I \mathcal{L} V$ of degree zero. Put

$$
j_{2}=e^{\alpha} j_{1}
$$

One sees easily that $j_{1} \coprod j_{2}:(\mathcal{L} V, \delta) \coprod(\mathcal{L} V, \delta) \rightarrow(I \mathcal{L} V, D)$ injects and that $I \mathcal{L} V \approx \mathcal{L} V \coprod \mathcal{L} V \coprod \mathcal{L}(s V)$ as Lie algebras. 
Analogous to Proposition 28 is

Lemma 29. Let $f, g:(L, \delta) \rightarrow\left(L^{\prime}, \delta^{\prime}\right)$ be two DG-Lie algebra homomorphisms. Then $f \sim g$ if and only if $f \coprod g$ factors (in DLA) through the inclusion $j_{1} \coprod j_{2}$ of $L$ into its Tanre cylinder.

We state here the Lemaire-Aubry theorem, for proof see [5].

Proposition 30. Let $f, g:(L, \delta) \rightarrow\left(L^{\prime}, \delta^{\prime}\right)$ be two DG-Lie algebra homomorphisms. Then $f \sim g$ if and only if $U f \sim U g$ in $D G A$.

In any category with a concept of homotopy satisfying Lemma 27, the morphism $f: A \rightarrow B$ is called a homotopy equivalence if there exists $g: B \rightarrow A$ for which $g f \sim 1_{A}$ and $f g \sim 1_{B}$. Two objects have the same homotopy type if there is a homotopy equivalence between them.

Let $\varepsilon: A \rightarrow R$ be an augmentation for a graded algebra $(A, d, \mu)$ and put $\pi_{1}=$ $(1 \otimes \varepsilon): A \otimes A \rightarrow A, \pi_{2}=(\varepsilon \otimes 1): A \otimes A \rightarrow A$.

Definition 31. Let $(A, d, \mu)$ be a DG-algebra and $\psi:(A, d, \mu) \rightarrow(A, d, \mu) \otimes$ $(A, d, \mu)$ be a DG-algebra homomorphism. The quartet $(A, d, \mu, \psi)$ is called a Hopf algebra up to homotopy (henceforth Hah) over $R$ if there exist four homotopies in $D G A$

(i) between $\pi_{1} \psi$ and 1 ;

(ii) between $\pi_{2} \psi$ and 1 ;

(iii) between $(\psi \otimes 1) \psi$ and $(1 \otimes \psi) \psi$.

(iv) between $T \psi$ and $\psi$;

A homomorphism of Hah's is a DG-algebra homomorphism $f:(A, d, \mu) \rightarrow$ $\left(A^{\prime}, d^{\prime}, \mu^{\prime}\right)$ such that $(f \otimes f) \psi \sim \psi^{\prime} f$. The category of Hah's over $R$ and their homomorphisms is denoted by $H A H(R)$, or simply $H A H$.

A $\operatorname{Hah}(A, d, \mu, \psi)$ is called free, $r$-reduced, etc. if $(A, d, \mu)$ is so in $D G A$. A homomorphism in $H A H$ is called surjection, quasi-isomorphism, etc. if it is so in $D G A$. 
Let us denote by $H A H_{1}$ full subcategory of $H A H$ consisting of free 1-reduced objects, i.e. such $A$ 's which are isomorphic to $T V$ for some $V$ with $V_{0}=0$.

The cylinder and homotopy in $H A H$ is defined by essentially copying the definitions for these concepts in $D G A$. Let $(A, d, \mu, \psi) \in H A H$. Given any $D G A$ cylinder $\left(A^{*}, d^{*}, \mu^{*}\right)$ on the underlying DG-algebra $(A, d, \mu)$, put $\psi^{*}=\left(j_{1} \otimes j_{2}\right) \psi p$. Then $j_{1}$, $j_{2}$, and $p$ are automatically $H A H$ homomorphisms. The following two lemmas are applications of this definitions.

Lemma 32. Let $f:(A, d, \psi, \mu) \rightarrow\left(A^{\prime}, d^{\prime}, \psi^{\prime}, \mu^{\prime}\right)$ be an HAH homomorphism and let $g:(A, d, \mu) \rightarrow\left(A^{\prime}, d^{\prime}, \mu^{\prime}\right)$ be a DGA Homomorphism. The following are equivalent:

(i) $f \sim g$ in $D G A$;

(ii) $g$ is a $H A H$ homomorphism and $f \sim g$ in $H A H$.

Lemma 33. Let $f:(A, d, \psi, \mu) \rightarrow\left(A^{\prime}, d^{\prime}, \psi^{\prime}, \mu^{\prime}\right)$ be an HAH homomorphism and suppose $g:\left(A^{\prime}, d^{\prime}, \mu^{\prime}\right) \rightarrow(A, d, \mu)$ is a homotopy inverse in $D G A$. Then $g \in H A H$, and $f$ and $g$ are homotopy inverses in $H A H$.

We are ready now to state some theorems from $[\mathbf{3}]$. We state the results over the rationals, although they were proved for any $R \not \supset Q$ under certain restrictions on $r$-mildness of given $D G$-objects.

Proposition 34. Let $(A, d)$ be a free $D G$-algebra, $A=T V$ with $V_{0}=0$. Let $\psi$ be a coproduct on $A$ which is a DG-algebra homomorphism. Suppose $\psi$ is a both cocommutative and coassociative. Then there is a graded $Q$-module $W$ isomorphic to the graded $Q$-module $V$ such that $(A, d)=U(\mathcal{L W}, \mathrm{d})$ and $\psi=\Delta_{\mathcal{L W}}$, where $(\mathcal{L} \mathrm{W}, \mathrm{d})$ is a free Lie algebra.

Let introduce some notations which we need in the definitions below.

Let $\mathcal{L} \mathrm{V}$ be a free Lie algebra over $V$. Let $\sigma \in S_{k}$ and let $\underline{x}=\left(x_{1}, \cdots, x_{k}\right)$ be a list of (homogeneous) elements in $\mathcal{L} \mathrm{V}$. Define the graded sign $\sigma: \underline{x}$ of $\sigma$ relative to $\underline{x}$ to be the sign (i.e. +1 or -1 ) that $\sigma$ has when viewed as permuting $k$-blocks of sizes 
$\left|x_{1}\right|, \cdots,\left|x_{k}\right|$. Put

$$
C(\underline{x})=C\left(x_{1}, \cdots, x_{k}\right)=\frac{1}{k !} \Sigma_{\sigma \in S_{k}}[\sigma: \underline{x}] x_{\sigma(1)} \otimes \cdots \otimes x_{\sigma(k)}
$$

which defines an element in $T(V)=U \mathcal{L}(\mathrm{V})$. Let $U_{0}(V)=R$ and $U_{1}(V)=\mathcal{L}(\mathrm{V})$ and

$$
U_{n}(V)=\operatorname{span}\left\{C\left(x_{1}, \cdots, x_{k}\right) \mid x_{i} \in \mathcal{L}(\mathrm{V})\right\} \in \mathrm{T}(\mathrm{V}) .
$$

Define a map $\mu: I T(V) \otimes I T(V) \rightarrow I T(V)$ by

$$
\mu\left(x_{1} \otimes C\left(x_{2}, \cdots, x_{k}\right)\right)= \begin{cases}\frac{1}{k} C\left(x_{1}, \cdots, x_{k}\right) & \text { for } k \geq 1 \\ 0 & \text { otherwise }\end{cases}
$$

Put $E=I T(V)^{\otimes 2} \oplus I T(V)^{\otimes 3}$ and let $\phi=\left(\phi_{1}, \phi_{2}\right): T(V)^{\otimes 2} \rightarrow E$, where $\phi_{1}=1-T$ and $\phi_{2}=I\left(\Delta_{s h}\right) \otimes 1-1 \otimes I\left(\Delta_{s h}\right)$. Define $\lambda: E \rightarrow T(V)^{\otimes 2}$ as follows

$$
\begin{cases}\lambda(a \otimes b)=-\frac{i}{k} T(a \otimes b) & a \in V^{\otimes i}, b \in V^{\otimes k-i} ; \\ \lambda(a \otimes b \otimes c)=\frac{i+j}{k}(1+T)(\mu(a \otimes b) \otimes c) & a \in V^{\otimes i}, b \in V^{\otimes j}, c \in V^{\otimes k-i-j}\end{cases}
$$

Let $q: T(V) \rightarrow I T(V)$ denote natural projection. We also allow $q$ to denote any of the homomorphisms $q^{\otimes 2}: T(V)^{\otimes 2} \rightarrow I T(V)^{\otimes 2}, q^{\otimes 3}: T(V)^{\otimes 3} \rightarrow I T(V)^{\otimes 3}$, $q^{\otimes 2} \oplus q^{\otimes 3}: T(V)^{\otimes 2} \oplus T(V)^{\otimes 3} \rightarrow I T(V)^{\otimes 2} \oplus I T(V)^{\otimes 3}=E$.

Definition 35. Let $(A, d, \mu, \psi)$ be a Hah, $A=T(V), V_{0}=0$. Call $(A, d, \mu, \psi)$ $n$-perfect if

(i) $(\varepsilon \otimes 1) \psi=1=(1 \otimes \varepsilon) \psi$

(ii) $\psi=\psi T$ and $(\psi \otimes 1) \psi=(1 \otimes \psi) \psi$ on $V_{\leq n}$

(iii) there exist derivation homotopies $G_{1}$ from $\psi$ to $\psi T$ and $G_{2}$ from $(\psi \otimes 1) \psi$ to $(1 \otimes \psi) \psi$ such that $q\left(G_{1}, G_{2}\right)\left((k e r J(\psi))_{<n}\right) \subseteq i m\left(1_{E}-\phi \lambda\right) \subseteq E$.

Lemma 36. Let $(A, d, \mu, \psi)$ be a n-perfect Hah, $A=T(V), V_{0}=0$. Then there exist $\chi \sim \psi$ such that $\chi$ coincides with $\psi$ on $T\left(V_{\leq n}\right)$ and $(A, d, \mu, \chi)$ is $n+1$-perfect. Furthermore, the derivation homotopy $F$ from $\chi$ to $\psi$ may be chosen so that $F\left(V_{i}\right)=0$ for $i<n$ and for $i>n+1$.

The initial step of the induction is provided by 
Proposition 37. Let $(A, d, \mu, \psi)$ be a Hah. Then there exists $\chi \sim \psi$ such that $(A, d, \mu, \chi)$ is 1-perfect.

We combine Proposition 34, Lemma 36 and Proposition 37 to obtain the following (over the rationals)

Proposition 38. Let $(A, d, \mu, \psi)$ be a Hah, $A=T(V), V_{0}=0$. Then there exists on $A$ a strictly coassociative and strictly cocommutative diagonal $\chi \sim \psi$; moreover, the resulting $D G$-Hopf algebra $(A, d, \mu, \chi)$ has the form $U \mathcal{L}(W, d)$ for some $Q$-module $W$.

We proceed with a definition, and inductive step, which are analogs of the definition and lemma above.

Definition 39. Let $(\mathcal{L} V, d)$ and $(M, e)$ be DG-Lie algebras with $V_{0}=0$ and $M_{0}=0$. An $H A H$ homomorphism $f: U(\mathcal{L} V, d) \rightarrow U(M, e)$ is n-perfect if

(i) $f\left(V_{\leq n}\right) \subset M ;$ and

(ii) there is a derivation homotopy $G$ from $\Delta_{U M} f$ to $(f \otimes f) \Delta_{\mathcal{L} V}$ such that $q G\left(V_{<n}\right) \subseteq \operatorname{Im}\left(1-I\left(\Delta_{U M}\right) \mu\right) \subseteq I(U M)^{\otimes 2}$.

REMARK 40. Any such $f$ is automatically 1-perfect from the dimensional viewpoint $[3]$.

Lemma 41. Let $(\mathcal{L} V, d)$ and $(M, e)$ be DG-Lie algebras with $V_{0}=0, M_{0}=0$ and let $f: U(\mathcal{L} V, d) \rightarrow U(M, e)$ be n-perfect. There is an $(n+1)$-perfect $g$ such that $g \sim f$. If $f$ is an isomorphism, so is $g$. There is a derivation homotopy $F$ from $f$ to $g$ having $F\left(V_{i}\right)=0$ for $i<n$ and for $i>n+1$.

Note that if the $H A H$ homomorphism $f: U(\mathcal{L V}, \mathrm{d}) \rightarrow U(M, e)$ is $n$-perfect it follows from $(i)$ that $(f \otimes f) \Delta_{U \mathcal{L}(\mathrm{V})}=\Delta_{U M} f$ in dimensions $\leq n$; this implies that if $f: U(\mathcal{L V}) \rightarrow U(M)$ is $n$-perfect for any $n \in N$ then it is strictly comultiplicative on all of $U(\mathcal{L} \mathrm{V})$. Hence applying Proposition 41 inductively we obtain the following (over the rationals) 
Proposition 42. Let $f: U(\mathcal{L} V, d) \rightarrow U\left(\mathcal{L} W\right.$, e) be a Hah homomorphism, $V_{0}=$ $W_{0}=0$. Then there exists a DG-Hopf algebra map $g: U(\mathcal{L} V, d) \rightarrow U(\mathcal{L} W, e)$ such that $g \sim f$. If $f$ is an isomorphism so is $g$.

Let us briefly discuss homotopy categories. For any category $\mathcal{C}$ having a notion of homotopy between morphisms satisfying Lemma 27, we may construct the classical homotopy category $\mathcal{H o C}$. Objects in $\mathcal{H o C}$ are objects of $\mathcal{C}$, but morphisms in $\mathcal{H o C}$ are homotopy classes of morphisms in $\mathcal{C}$. Two objects of the same homotopy type in $\mathcal{C}$ become isomorphic in $\mathcal{H} o \mathcal{C}$. A functor $\mathcal{F}: \mathcal{C} \rightarrow \mathcal{D}$ such that $\mathcal{F} f \sim \mathcal{F} g$ whenever $f \sim g$ induces a functor $\mathcal{H} \circ \mathcal{F}: \mathcal{H o C} \rightarrow \mathcal{H} o \mathcal{D}$. In particular, the functor $U$ of Subsection 1.8 (universal enveloping algebra functor) induces $\mathcal{H} o U$.

A functor $\mathcal{F}: \mathcal{C} \rightarrow \mathcal{D}$ is an equivalence of categories if it induces a bijection on isomorphic classes of objects, as well as bijections $\operatorname{MOR}_{\mathcal{C}}(A, B) \rightarrow M O R_{\mathcal{D}}(\mathcal{F} A, \mathcal{F} B)$ for each pair of objects in $\mathcal{C}$. Let us denote by $D L A_{1}(Q)$ the category of 1 reduced $D G$-Lie algebras which are free as Lie algebras. We are ready to state the principal result of $[\mathbf{3}]$.

Theorem 43. $\mathcal{H o}(U): \mathcal{H o D L A} A_{1}(Q) \rightarrow \mathcal{H}_{o H A H_{1}}(Q)$ is an equivalence. Furthermore, the functor $U$ itself induces a bijection on isomorphism classes of objects.

Anick proved this theorem in several intermediate steps, see [3]. We state below only one of the steps (theorem 6.4 in [3]) and refer to it in the last section.

Theorem 44. Let $f:\left(A, d_{A}, \psi_{A}, \mu_{A}\right) \rightarrow\left(B, d_{B}, \psi_{B}\right)$ be any homomorphism in $H A H_{1}(Q)$. There exists a homomorphism $\alpha:\left(L, d_{L}\right) \rightarrow\left(M, d_{M}\right)$ in $D L A_{1}(Q)$ such that $U\left(L, d_{L}\right)$ may be identified with $\left(A, d_{A}, \mu_{A}\right) ; U\left(M, d_{M}\right)$ may be identified with $\left(B, d_{B}, \mu_{B}\right)$; and there exist $D G A$ homotopies $f \sim U \alpha, \psi_{A} \sim \Delta_{L}, \psi_{B} \sim \Delta_{M}$. Furthermore, $\alpha$ is an isomorphism if and only if $f$ is an isomorphism.

We will state below another fundamental theorem from [4], which is a new proof of the fundamental theorem of rational homotopy theory. The fundamental theorem of rational homotopy theory asserts that the homotopy category $\mathcal{H}_{o} \operatorname{Top}_{1}(Q)$ of simply 
connected rational spaces is equivalent to the homotopy category $\mathcal{H} o D L A_{1}(Q)$ of connected differential graded Lie Q-algebras [38]. Letting $\mathcal{Q}(X)$ be a Quillene's minimal Lie algebra model for simply connected space $X$, a rephrasing of this could say that a model $\mathcal{Q}$ induces a bijection on weak rational homotopy types, and that two maps $f, g: X \rightarrow Y$ are $Q$-locally homotopic if and only if $\mathcal{Q}(f) \sim \mathcal{Q}(g)$ in $D L A_{1}(Q)$. Consequently any statement or problem about $\mathcal{H o T o p}_{1} Q$ may be translated into an equivalent statement in the purely algebraic category $D L A_{1}(Q)$. The model $\mathcal{Q}$ is called a "complete rational homotopy invariant" on simply connected spaces.

Let $C W_{1}$ denote the category of simply connected $C W$ complexes, with arbitrary pointed maps among them. Anick constructed a model $\mathcal{L}$ from $C W_{1}$ to the category $D L A_{1}$ of 1-reduced differential graded Lie algebras [3]. Denote by $\mathcal{H} o C W_{1} Q$ the homotopy category of simply connected $C W$ complexes by collapsing $Q$-local homotopy classes of maps and by $\mathcal{H} o D L A_{1}(Q)$ the homotopy category of 1-reduced $D G$ - Lie algebras as in Theorem 40. Then

TheOREM 45. The model $\mathcal{L}$ induces an equivalence of rational homotopy categories

$$
\mathcal{H o L}: \mathcal{H}_{o} C W_{1}(Q) \longrightarrow \mathcal{H o D L A}_{1}(Q)
$$

\subsection{Homotopy G-ALGeBRA}

We start this subsection with the definition of Homotopy G-algebra. We slightly change the notation using the sequence of operations $E_{1, q}: A \otimes\left(\otimes^{q} A\right) \rightarrow A$ instead of sequences of multibraces $a\left\{a_{1}, \cdots, a_{q}\right\}$ originally used in $[\mathbf{1 7}]$.

Definition 46. A Homotopy G-algebra is defined as a DG-algebra $(A, d, \mu)$ together with a set of operations

$$
E=\left\{E_{0,1}=1, E_{1, q}: A \otimes\left(\otimes^{q} A\right) \rightarrow A, q=0,1,2, \cdots\right\}
$$


satisfying

(i) $d E_{1, q}\left(a \mid b_{1}, \cdots, b_{q}\right)-E_{1, q}\left(d a \mid b_{1}, \cdots, b_{q}\right)-$

$$
\begin{gathered}
(-1)^{|a|} \sum_{j=1}^{q}(-1)^{\left|b_{1}\right|+\cdots+\left|b_{j-1}\right|} E_{1, q}\left(a \mid b_{1}, \cdots, d b_{j}, \cdots, b_{q}\right)= \\
=(-1)^{|a|+1} \sum_{i=1}^{q-1}(-1)^{\left|b_{1}\right|+\cdots+\left|b_{i}\right|} E_{1, q-1}\left(a \mid b_{1}, \cdots, b_{i} \cdot b_{i+1}, \cdots, b_{q}\right)+ \\
(-1)^{(|a|+1)\left(\left|b_{1}\right|\right)} b_{1} \cdot E_{1, q-1}\left(a \mid b_{2}, \cdots, b_{q}\right)+ \\
(-1)^{|a|+\left|b_{1}\right|+\cdots+\left|b_{q-1}\right|} E_{1, q-1}\left(a \mid b_{1}, \cdots, b_{q-1}\right) \cdot b_{q} ;
\end{gathered}
$$

(ii) $E_{1, q}\left(a_{1} \cdot a_{2} \mid b_{1}, \cdots, b_{q}\right)=$

$$
=\sum_{k=0}^{q}(-1)^{\varepsilon} E_{1, k}\left(a_{1} \mid b_{1}, \cdots, b_{k}\right) \cdot E_{1, q-k}\left(a_{2} \mid b_{k+1}, \cdots, b_{q}\right) \text {; }
$$

(iii) $E_{1, n}\left(E_{1, m}\left(a \mid b_{1}, \cdots, b_{m}\right) \mid c_{1}, \cdots, c_{n}\right)=$

$$
\begin{array}{r}
=\sum_{0 \leq i_{1} \leq \cdots \leq i_{m} \leq n}(-1)^{\tau} E_{1, .}\left(a \mid c_{1}, \cdots, c_{i_{1}}, E_{1, .}\left(b_{1} \mid c_{i_{1}+1}, \cdots\right), \cdots\right. \\
\left.\cdots, c_{i_{m}}, E_{1, .}\left(b_{m} \mid c_{i_{m}+1}, \cdots\right), \cdots, c_{n}\right)
\end{array}
$$

where $\varepsilon=\left(\left|a_{2}\right|+1\right) \sum_{i=1}^{k}\left|b_{i}\right|$, and $\tau=\sum_{p=1}^{m}\left|b_{p}\right| \sum_{q=1}^{i_{p}}\left|c_{q}\right|$.

It follows from $(i)$ and $(i i)$ that the map

$$
E:\left(B A \otimes B A, d_{B A \otimes B A}, \Delta_{\otimes}\right) \rightarrow(A, d, \mu)
$$

given by

$$
\left.E\right|_{\left(\otimes^{p} A\right) \otimes\left(\otimes^{q} A\right)}=\left\{\begin{array}{cl}
1, & \text { if } p=0, q=1 \\
E_{1, q}, & \text { if } p=1 \\
0, & \text { otherwise }
\end{array}\right.
$$

is a twisting cochain $d E+E d_{B A \otimes B A}=E \smile E$, which, according to Subsections 1.7 and 1.11, uniquely defines a $D G$-coalgebra homomorphism $\mu_{E}:\left(B A \otimes B A, d_{B A \otimes B A}, \Delta_{\otimes}\right) \rightarrow\left(B A, d_{B A}, \Delta\right)$ by

$$
\mu_{E}\left(b_{1}, \ldots, b_{m} \mid c_{1}, \ldots, c_{n}\right)=
$$




$$
\begin{array}{r}
\sum_{0 \leq i_{1} \leq \cdots \leq i_{m} \leq n}(-1)^{\varepsilon} c_{1}, \cdots, c_{i_{1}}, E_{1, .}\left(b_{1} \mid c_{i_{1}+1}, \cdots\right), \cdots \\
\left.\cdots, c_{i_{m}}, E_{1, .}\left(b_{m} \mid c_{i_{m}+1}, \cdots\right), \cdots, c_{n}\right)
\end{array}
$$

where $\varepsilon=\sum_{p=1}^{m}\left|b_{p}\right| \sum_{q=1}^{i_{p}}\left|c_{q}\right|$. The third condition implies that this product is associative.

Thus the bar-construction $\left(B A, d_{B A}, \Delta, \mu_{E}\right)$ over $\left(A, d, \mu,\left\{E_{1, q}\right\}\right)$ is a $D G$-Hopf algebra.

For a 1-reduced simplicial set $X$, i.e. $X_{0}=X_{1}=\{*\}$, Baues has constructed a $D G$ Hopf algebra map $\phi: \Omega C_{*}(X, R) \rightarrow \Omega C_{*}(X, R) \otimes \Omega C_{*}(X, R)$, see [6]; Gerstenhaber and Voronov Showed that dualization of the restrictions

$$
\phi i_{1}: C_{*}(X, R) \rightarrow \Omega C_{*}(X, R) \rightarrow \Omega C_{*}(X, R) \otimes \Omega C_{*}(X, R)
$$

yields to a Homotopy G-algebra structure on singular cochains $C^{*}(X, R)$, see corollary 6 in [18]. We describe these dual operations below.

For an n-simplex $\sigma: \triangle(n) \rightarrow X$ with $\triangle(n)$ being the standard n-simplex, let $\sigma\left(n_{0}, \cdots, n_{k}\right)$ denotes its face spanned by the vertices $n_{0}, \cdots, n_{k}$, where $i \mapsto n_{i}$ is a monotonic function $\triangle(k) \rightarrow \triangle(n)$. The singular cochain complex $C^{*}(X, R)$ has a natural Homotopy G-algebra structure; suppose $\varphi \in C^{m_{0}}(X, R), \varphi_{i} \in C^{j_{i}}(X, R)$, $\sigma \in \triangle^{m_{0}+j_{1}+\cdots+j_{k}-k}(X)$, then

$$
\begin{gathered}
E_{1, k}\left(\varphi \mid \varphi_{1}, \cdots, \varphi_{k}\right) \sigma= \\
\sum_{n_{1}, \cdots, n_{k}}(-1)^{\varepsilon} \varphi \sigma\left(0, \cdots, n_{1}, n_{1}+j_{1}, \cdots, n_{2}, \cdots, n_{k}+j_{k}, \cdots, n_{k+1}=m\right) \\
\varphi_{1} \sigma\left(n_{1}, \cdots, n_{1}+j_{1}\right) \varphi_{2} \sigma\left(n_{2}, \cdots, n_{2}+j_{2}\right) \cdots \varphi_{k} \sigma\left(n_{k}, \cdots, n_{k}+j_{k}\right)
\end{gathered}
$$

where $\varepsilon=\sum_{i=1}^{k+1}(i-1)\left(n_{i}+1-\left(n_{i-1}+j_{i-1}\right)\right)$. Note that $E_{1,1}$ coincides with the Steenrod's $\smile_{1}$ product. For more details see [6], see also [28].

Once we have a Homotopy G-algebra structure $\left\{E_{1, k}\right\}$ on $C^{*}(X, R)$, we know that the Bar construction $\left(B C^{*}(X, R), d_{C^{*}(X, R)}, \Delta\right)$, together with the multiplication $\mu_{E}$ defined above, becomes a $D G$ Hopf algebra. 
Later in [7] it was shown that the diagonal $\phi: \Omega C_{*}(X, R) \rightarrow \Omega C_{*}(X, R) \otimes$ $\Omega C_{*}(X, R)$ is cocommutative up to homotopy; i.e. there is a derivation homotopy $\psi: \Omega C_{*}(X, R) \rightarrow \Omega C_{*}(X, R) \otimes \Omega C_{*}(X, R)$ such that $\psi: \phi \sim T \phi$. Baues described the homotopy $\psi$ explicitly on page 475 . So $\left(\Omega C_{*}(X, R), d_{C_{*}(X, R)}, \mu, \phi\right)$ is a Hopf algebra up to homotopy $(H a h)$; indeed, we have equalities not just homotopies for first 3 axioms of Definition 31.

We have seen above that the Baues diagonal $\phi$ on $\Omega C_{*}(X, R)$ canonically corresponds to a multiplication $\mu_{E}$ on $B C^{*}(X, R)$. Similarly the direct formula of homotopy $\psi$ and its implications, as it was hypothesized by Baues (see page 476 in [7]), has the dual analogs in the coalgebraic case, i.e. on $B C^{*}(X, R)$.

Let us describe the "dual" homotopy in the coalgebraic case. Suppose that $f_{k} \in C^{i_{k}}(X, R), g_{t} \in C^{j_{t}}(X, R), \sigma \in C_{m}(X, R), m=\sum_{k=1}^{p} i_{k}+\sum_{t=1}^{q} j_{t}-p-q$, then the set of operations

$$
\begin{gathered}
F_{p, q}\left(f_{1}, \cdots, f_{p} \mid g_{1}, \cdots, g_{q}\right) \sigma= \\
\sum_{n_{1}, \cdots, n_{q}}(-1)^{\varepsilon} f_{1} \sigma\left(0, \cdots, n_{1}, n_{1}+j_{1}, \cdots, n_{2}, \cdots, n_{q-1}+j_{q-1}, \cdots, n_{q}, k_{1}, \cdots, n_{q+1}\right) \\
k_{1}, \cdots, k_{p} \\
g_{1} \sigma\left(n_{1}, \cdots, n_{1}+j_{1}\right) g_{2} \sigma\left(n_{2}, \cdots, n_{2}+j_{2}\right) \cdots g_{q-1} \sigma\left(n_{q-1}, \cdots, n_{q-1}+j_{q-1}\right) \\
g_{q} \sigma\left(n_{q}, \cdots, k_{1}, n_{q+1}, \cdots, k_{2}, k_{2}+i_{2}, \cdots, k_{3}, \cdots, k_{p}+i_{p}, \cdots, k_{p+1}=m\right) \\
f_{2} \sigma\left(k_{2}, \cdots, k_{2}+i_{2}\right) f_{3} \sigma\left(k_{3}, \cdots, k_{3}+i_{3}\right) \cdots f_{p} \sigma\left(k_{p}, \cdots, k_{p}+i_{p}\right),
\end{gathered}
$$

where $\varepsilon=\sum_{t=2}^{q}(t-1)\left(n_{t}+1-\left(n_{t-1}+j_{t-1}\right)\right)+q\left(n_{q+1}+1-k_{1}\right)+\left(k_{2}+1-n_{q+1}\right)+$ $\sum_{t=3}^{p+1}\left(k_{t}+1-\left(k_{t-1}+i_{t-1}\right)\right)$, uniquely defines a coderivation homotopy $D_{F}$ so that $D_{F}: \mu_{E} \sim \mu_{E} T$.

Thus the Bar construction $\left(B C^{*}(X, R), d_{B C^{*}(X, R)}, \Delta, \mu_{E}\right)$ on $C^{*}(X, R)$ is a coalgebraic Hah. By the coalgebraic Hah, of course, we mean $D G$-coalgebra $(C, d, \Delta)$ together with a $D G$-coalgebra map $\mu: C \otimes C \rightarrow C$ such that the coaugmentation $\eta$ 
of coalgebra $C$ is a two sided homotopy unit for $\mu, \mu(\eta \otimes 1) \sim 1$ and $\mu(1 \otimes \eta) \sim 1$, and $\mu$ is homotopy associative, $\mu(\mu \otimes 1) \sim \mu(1 \otimes \mu)$, and homotopy commutative, $\mu \sim \mu T$. A homomorphism $f:(C, d, \Delta, \mu) \rightarrow\left(C^{\prime}, d^{\prime}, \Delta^{\prime}, \mu\right)$ between two such objects is a $D G$-coalgebra map $f:(C, d, \Delta) \rightarrow\left(C^{\prime}, d^{\prime}, \Delta^{\prime}\right)$ such that $f \mu \sim \mu^{\prime}(f \otimes f)$; a homomorphism $f$ is called a quasi-isomorphism, isomorphism etc. if it is so in $D G C$. Throughout the rest of this paper we work with free coalgebras, $C \cong T^{c} V$, and coderivation homotopies. The coalgebraic Hah's and their homomorphisms form a category denoted by $H A H$ (from the context it always will be clear whether the given objects belong to the algebraic or coalgebraic $H A H$, thus the notation $H A H$ is unambiguous).

We have the coalgebraic analogs of the algebraic theorems of Subsection 1.13. In particular, the dual to the Propositions 38 and 42 are the following statements:

Proposition 47. Let $(C, d, \Delta, \mu)$ be a coalgebraic Hah, $C=T^{c}(V), V_{0}=0$. Then there exists on $C$ strictly associative and strictly commutative multiplication $\phi \sim \mu$; moreover, the resulting DG-Hopf algebra $(C, d, \Delta, \phi)$ has the form $U^{c} \mathcal{L}^{c}(W, d)$ for some $Q$-module $W$.

Proposition 48. Let $f: U^{c}\left(\mathcal{L}^{c} V, d\right) \rightarrow U^{c}\left(\mathcal{L}^{c} W, e\right)$ be a Hah homomorphism, and $V_{0}=W_{0}=0$. Then there exists a DG-Hopf algebra map $g: U^{c}\left(\mathcal{L}^{c} V, d\right) \rightarrow$ $U^{c}\left(\mathcal{L}^{c} W, e\right)$ such that $g \sim f$. If $f$ is an isomorphism so is $g$.

Definition of the homomorphism in $H A H$ motivates the following

Definition 49. A Homotopy G-algebra morphism

$$
f:\left(A, d_{A}, \mu_{A},\left\{E_{1, q}^{A}\right\}\right) \rightarrow\left(B, d_{B}, \mu_{B},\left\{E_{1, q}^{B}\right\}\right)
$$

is a DASH map

$$
f:\left(A, d_{A}, \mu_{A}\right) \rightarrow\left(B, d_{B}, \mu_{B}\right)
$$

such that the coalgebra map

$$
B f:\left(B A, d_{B A}, \mu_{E^{A}}, \Delta\right) \rightarrow\left(B B, d_{B B}, \mu_{E^{B}}, \Delta\right)
$$


of appropriate bar constructions is multiplicative up to homotopy, i.e. there exists a coderivation homotopy from $B f \mu_{E^{A}}$ to $\mu_{E^{B}}(B f \otimes B f)$.

A Homotopy G-algebra morphism

$$
f:\left(A, d_{A}, \mu_{A},\left\{E_{1, q}^{A}\right\}\right) \rightarrow\left(B, d_{B}, \mu_{B},\left\{E_{1, q}^{B}\right\}\right)
$$

is called quasi-isomorphism, isomorphism, etc. if $B f$ is so in $D G C$.

Clearly, the identity map and a composition of Homotopy $G$-algebra morphisms are Homotopy $G$-algebra morphisms. Thus Homotopy $G$-algebras and their morphisms form a category which we call Homotopy $G$-algebra category. 


\section{On the Gugenheim MAP}

In this section we give some applications of the Baues operations $\left\{E_{1, k}\right\}$ and $\left\{F_{p, q}\right\}$.

Let $X$ be a simplicial complex ( $C^{\infty}$ manifold). Then there are two $D G$-algebras, Sullivan's algebra of rational differential forms (de Rham algebra of differential forms) $A^{*}(X, Q)[\mathbf{1 6}]$ and the algebra of normalized simplicial cochains (normalized differentiable cochains), $C^{*}(X, Q)$ corresponding to $X$.

The transformation $\rho: A^{*}(X, Q) \rightarrow C^{*}(X, Q)$ given by

$$
<\rho \omega, c>=\int_{c} \omega,
$$

where $c$ is any simplicial (singular) chain, was first constructed in the 1930's in the context of smooth manifolds and ordinary differential forms. The de Rham theorem says, whether $X$ is a smooth manifold or simplicial complex, that $\rho$ induces a homology isomorphism

$$
H \rho: H A^{*}(X, Q) \rightarrow H C^{*}(X, Q)
$$

which is a map of algebras [13].

Although $\rho$ itself is not a map of algebras $\left(A^{*}(X, Q)\right.$ is commutative, $C^{*}(X, Q)$ is not) it was shown in [20] that it can be extended to a map of $D A S H$; i.e. there exists a whole family of higher operations

$$
\rho_{i}: A^{*}(X, Q)^{\otimes i} \rightarrow C^{*}(X, Q)
$$


such that

$$
D \rho_{i}=\sum_{j=1}^{i-1}(-1)^{j}\left\{\left(\rho_{j} \smile \rho_{i-j}\right)-\rho_{i-1}\left(1^{(i-j)} \otimes \wedge \otimes 1^{(i-j-1)}\right)\right\} .
$$

For $i=1,2$ we get

$$
D \rho_{1}=0, \quad D \rho_{2}=\rho_{1} \wedge-\rho_{1} \smile \rho_{1} .
$$

The latter statement contains, of course, the classical result that $H \rho$ is multiplicative.

From Subsection 1.10 we know that a given $D A S H$ map $\left\{\rho_{i}\right\}$ canonically defines a coalgebra map

$$
B\left\{\rho_{i}\right\}:\left(B A^{*}, d_{B A^{*}}, \triangle\right) \rightarrow\left(B C^{*}, d_{B C^{*}}, \triangle\right)
$$

of appropriate bar constructions; but both $\left(B A^{*}(X, Q), d_{B A^{*}(X, Q)}, \triangle\right)$ and $\left(B C^{*}(X, Q), d_{B C^{*}(X, Q)}, \triangle\right)$ are $D G$-Hopf algebras with respect to shuffle $\mu_{s h}$ and Baues $\mu_{E}$ products respectively. We showed in [29] that

THEOREM 50. Gugenheim's map

$$
B\left\{\rho_{i}\right\}:\left(B A^{*}(X, Q), d_{B A^{*}(X, Q)}, \triangle, \mu_{s h}\right) \rightarrow\left(B C^{*}(X, Q), d_{B C^{*}(X, Q)}, \triangle, \mu_{E}\right)
$$

is multiplicative up to homotopy.

Below we recall the construction of the Sullivan functor and state some key lemmas which we use to prove the theorem.

\subsection{Sullivan FUnCTOR AND SOME KEY LEMmaS}

We begin this subsection with short sketch of the Sullivan functor $A(K)$ mainly following [15], [32]. For more details see [16].

A simplicial object $K$ with values in a category $\mathcal{C}$ is a sequence $\left\{K_{n}\right\}_{n \geq 0}$ of objects in $\mathcal{C}$, together with $\mathcal{C}$-morphisms

$$
\mathfrak{d}_{i}: K_{n+1} \rightarrow K_{n}, \quad 0 \leq i \leq n+1 \quad \text { and } \quad s_{j}: K_{n} \rightarrow K_{n+1}, \quad 0 \leq j \leq n,
$$

called face and degeneracy operators, satisfying certain identities, see [34] for example. 
A simplicial morphism $f: K \rightarrow L$ between two such simplicial objects is a sequence of $\mathcal{C}$-morphisms $\varphi: K \rightarrow L$ commuting with $\mathfrak{d}_{i}$ and $s_{j}$.

A simplicial set $K$ is a simplicial object in the category of sets. Thus it consists of a sequence of sets $\left\{K_{n}\right\}_{n \geq 0}$ together with set maps.

A simplicial cochain algebra $A$ is a simplicial object in the category of cochain algebras: it consists of a sequence of cochain algebras $\left\{A_{n}\right\}_{n \geq 0}$ with appropriate face and degeneracy operators. Similarly simplicial cochain complexes, simplicial vector spaces ... are simplicial objects in the category of cochain complexes, vector spaces

\section{Construction of $\mathbf{A}(\mathrm{K})$.}

Let $K$ be a simplicial set, and let $A$ be a simplicial cochain complex or a simplicial cochain algebra. Then

$$
A(K)=\left\{A^{p}(K)\right\}_{p \geq 0}
$$

is the "ordinary" cochain complex (or cochain algebra) defined as follows:

- $A^{p}(K)$ is the set of simplicial set morphisms from $K$ to $A^{P}$.

Thus an element $\Phi \in A^{p}(K)$ is a mapping that assigns to each $n$-simplex $\sigma \in K_{n}$ $(n \geq 0)$ an

element $\Phi_{\sigma} \in\left(A^{p}\right)_{n}$, such that $\Phi_{\mathfrak{d}_{i} \sigma}=\mathfrak{d}_{i} \Phi_{\sigma}$ and $\Phi_{s_{j} \sigma}=s_{j} \Phi_{\sigma}$.

- Addition, the scalar multiplication and the differential are given by

$$
(\Phi+\Psi)_{\sigma}=\Phi_{\sigma}+\Psi_{\sigma}, \quad(\lambda \cdot \Phi)_{\sigma}=\lambda \cdot \Phi_{\sigma} \quad \text { and } \quad(d \Phi)_{\sigma}=d \Phi_{\sigma}
$$

- If $A$ is a simplicial cochain algebra multiplication in $A(K)$ is given by

$$
(\Phi \cdot \Psi)_{\sigma}=\Phi_{\sigma} \cdot \Psi_{\sigma}
$$

- If $\varphi: K \rightarrow L$ is a morphism of simplicial sets then $A(\varphi): A(L) \rightarrow A(K)$ is the morphism of

cochain complexes (or cochain algebras) defined by

$$
(A(\varphi) \Phi)_{\sigma}=\Phi_{\varphi \sigma}
$$


- If $\Theta: A \rightarrow B$ is a morphism of simplicial cochain complexes (or simplicial cochain algebras)

then $\Theta(K): A(K) \rightarrow B(K)$ is the morphism defined by

$$
(\Theta(K) \Phi)_{\sigma}=\Theta\left(\Phi_{\sigma}\right)
$$

- When $X$ is a topological space we write $A(X)$ for $A\left(S_{*}(X)\right)$ where $S_{\star}(X)=$ $\left\{S_{n}(X)\right\}_{n \geq 0}$ is simplicial set of singular simplices $\sigma: \triangle^{n} \rightarrow X$ on the topological space $X$.

REMARK 51. Note that the construction $A(K)$ is functorial in $A$ and contrafunctorial in $K$.

Let denote by $\triangle^{n}$ the Euclidean $n$-simplex $\left(\sum_{i=0}^{n} t_{i}=1, t_{i} \geq 0\right)$ in $R^{n+1}$ and by $\triangle^{n} \underset{\sigma_{i}}{\stackrel{\mathfrak{d}_{i}}{\rightleftarrows}} \triangle^{n+1}$ the ordinary coface and codegeneracy operators.

Let $\triangle$ be a category, objects of which are sets $\triangle^{n}$, and morphisms -compositions of $\mathfrak{d}_{i}$ and $\sigma_{i}$. The simplicial object in a category $\mathcal{B}$ we call any contravariant functor $K: \triangle \rightarrow \mathcal{B}$ (i.e. the family $\left(K_{n}, d_{i}, s_{i}\right)$ of objects and morphisms $K_{n-1} \leftarrow K_{n} \rightarrow K_{n+1}$ in $\mathcal{B}$ satisfying the ordinary relations of commutation); the simplicial morphism in $\mathcal{B}$ is a natural transformation of two such functors.

Denote by $A_{D R}\left(\triangle^{n}\right)$ the classical cochain algebra of real $C^{\infty}$ differential forms on $\triangle^{n}$. The (differentiable) maps $\mathfrak{d}_{i}$ and $\sigma_{i}$ induce operations

$$
A_{D R}\left(\triangle^{n-1}\right) \stackrel{d_{i}}{\leftarrow} A_{D R}\left(\triangle^{n}\right) \stackrel{s_{i}}{\rightarrow} A_{D R}\left(\triangle^{n+1}\right)
$$

so that the family $\left(A_{D R}\left(\triangle^{n}\right), d_{i}, s_{i}\right)$ is a commutative simplicial DGA; i.e. the simplicial object in commutative $D A$ over the reals. For the sake of simplicity we suppress $\triangle^{n}$ from notation, writing $A_{D R}$ for $A_{D R}\left(\triangle^{n}\right)$. The Poincare Lemma allows us to prove that each $A_{D R}\left(\triangle^{n}\right)=\left(A_{D R}\right)_{n}$ is acyclic. 
Denote by $A_{Q}$ a commutative simplicial $D G A$ (over rationals), where $\left(A_{Q}\right)_{n}$ is a subspace in $\left(A_{D R}\right)_{n}$ consisting of those differential forms

$$
\sum \omega_{i_{1} \cdots i_{p}} d t^{i_{1}} \wedge \cdots \wedge d t^{i_{p}}
$$

whose coefficients $\omega_{i_{1} \cdots i_{p}}$ in the barycentric coordinates $\left(t_{0}, t_{1}, \cdots t_{n}\right)$ are rational polynomials.

For a classical homotopy operator

$$
h: A_{D R}^{p}\left(\triangle^{n}\right) \rightarrow A_{D R}^{p-1}\left(\triangle^{n}\right),
$$

with $1_{\left(A_{D R}\right)_{n}^{p}}=d h+h d, h\left(A_{Q}\right)_{n}^{p} \subset\left(A_{Q}\right)_{n}^{p-1}$. Thus $\left(A_{Q}\right)_{n}$ is acyclic as well.

Definition 52. The Sullivan functor on a topological space $X$ is defined as a $D G A A^{*}(X, Q)$ with

$$
A^{p}(X, Q)=\operatorname{Hom}\left(S_{*}(X),\left(A_{Q}\right)^{p}\right),
$$

i.e. an element $\Phi \in A^{p}(X, Q)$ is a function assigning to each singular $n$-simplex of $X$ a polynomial $p$-form on $\triangle^{n}, n \geq 0$, compatible with the face and degeneracy maps (see construction of $A(K)$ at the beginning of current subsection). This motivates the following terminology: The commutative cochain algebra, $A^{p}(X, Q)$, is the cochain algebra of polynomial differential forms on $X$ over rationals.

Analogously over the reals, $A^{p}(X, R)=\operatorname{Hom}\left(S_{*}(X),\left(A_{D R}\right)^{p}\right)$.

\section{Acyclicity of the complex $\operatorname{Hom}\left(\mathrm{A}(\mathrm{X})^{\otimes i}, \mathrm{~A}(\mathrm{X})\right)$}

We continue with the following

Lemma 53. The Complex Hom $\left(A(X)^{\otimes i}, A(X)\right)$ is acyclic.

We shall use the machinery of contravariant acyclic models used by Gugenheim in $[\mathbf{2 0}]$; the machinery of acyclic models was introduced (covariantly) by Eilenberg and MacLane in [14]. 
For any contravariant functor $K: \mathcal{J} \rightarrow\{$ vector spaces $\}$ there is a new contravariant functor $\hat{K}: \mathcal{J} \rightarrow\{$ vector spaces $\}$

$$
\hat{K}(X)=\prod_{u: M \rightarrow X}\{K(M), u\}
$$

where the product is taken over all maps $u: M \rightarrow X$ of $\mathcal{J}$, and $M$ is a "model", i.e., one of the standard simplices $\triangle^{n}$. If $f: X \rightarrow Y$ is a map, then $\hat{K}(f): \hat{K}(Y) \rightarrow \hat{K}(X)$ is defined by

$$
\hat{K}(f)\left\{m_{v}, v\right\}=\left\{m_{f \cdot u}, u\right\},
$$

where $u: M \rightarrow X, v: M_{v} \rightarrow Y$, and $m_{v} \in K\left(M_{v}\right)$.

Let $K, L: \mathcal{J} \rightarrow \mathcal{V}$ be contravariant functors, and $\Theta: K \rightarrow L$ be a transformation of these functors. Then a new transformation $\hat{\Theta}: \hat{K} \rightarrow \hat{L}$ is defined by

$$
\hat{\Theta}(X)\{m, u\}=\{\Theta(M) m, u\}
$$

For any contravariant functor $K: \mathcal{J} \rightarrow \mathcal{V}$ define $\Phi: K \rightarrow \hat{K}$ by

$$
\Phi(X) h=\{K(u) h, u\} \quad(h \in K(X)) .
$$

We easily verify that

$$
\hat{\Theta} \Phi=\Phi \Theta
$$

In particular, for the functors $A^{*}$ and $C^{*}$ the functors $\hat{A}^{*}$ and $\hat{C}^{*}$ are defined by

$$
(\hat{A})^{n}=\widehat{\left(A^{n}\right)}, \quad(\hat{C})^{n}=\widehat{\left(C^{n}\right)},
$$

and we get differentials

$$
\hat{d}: \hat{A}^{*} \rightarrow \hat{A}^{*}, \quad \hat{d}: \hat{C} \rightarrow \hat{C}^{*}
$$

To prove Lemma 53 we need the following

Proposition 54. The Functor $A^{*}$ is corepresentable, i.e., there is a transformation of functors $\Psi: \hat{A}^{*} \rightarrow A^{*}$ such that $\Psi \Phi=$ the identity. 
Proof. We define $\Psi(X): \hat{A}^{*}(X) \rightarrow A^{*}(X)$ by

$$
<\Psi(X)\left\{m_{u}, u\right\}, v>=<m_{v}, 1_{M_{v}}>
$$

where $v: M_{v} \rightarrow X$, and $1_{M_{v}}$ denotes the identity map of $M_{v}$ regarded as a singular chain. Let $h \in A^{*}(X)$, then

$$
\begin{aligned}
<\Psi \Phi(h), v> & =<\Psi\left\{A^{*}(u) h, u\right\}, v>=<A^{*}(v) h, 1_{M_{v}}> \\
& =<h, v\left(1_{M_{v}}\right)>=<h, v>
\end{aligned}
$$

i.e. $\Psi \Phi=$ the identity.

We mentioned above that the functor $A^{*}$ is acyclic on models $M$. Moreover there is a homotopy operator (contraction)

$$
h_{M}: A^{*}(M) \rightarrow A^{*}(M)
$$

satisfying $d h_{M}+h_{M} d=1_{A^{*}(M)}$. It follows from the definition of the tensor product that the functors

$$
A^{* \otimes i}: A^{* \otimes i}(M) \rightarrow A^{* \otimes i}(M)
$$

are acyclic on models as well. Let us fix for each $i \geq 1$ and a model $M$, once and for all, a contraction

$$
h_{M}^{i}: A^{* \otimes i}(M) \rightarrow A^{* \otimes i}(M)
$$

In such a case Gugenheim in [20] defined a transformation of functors

$$
\hat{h}^{i}: \hat{A}^{* \otimes i}(X) \rightarrow \otimes^{i} \hat{A}^{* \otimes i}(X)
$$

by $\hat{h}_{M}^{i}\left\{\omega_{u}, u\right\}=\left\{h_{M}^{i} \omega_{u}, u\right\}$, where $u: M \rightarrow X$. Clearly, $D \hat{h}_{M}^{i}=1_{\otimes^{i} \hat{A}^{*}(M)}$.

Proof of Lemma 53. Thus we must to prove that if $D f=0$ for any $f \in$ $\operatorname{Hom}\left(A^{\otimes i}(X), A(X)\right), \operatorname{deg}(f)=k$, then there exists $g \in \operatorname{Hom}\left(A^{\otimes i}(X), A(X)\right)$ of degree $k-1$ satisfying $D g=f$. 
Let ue assume that $g$ is defined in dimension $<n$, i.e. on $\left(A^{\otimes i}(X)\right)_{<n}$, satisfying $d g_{j-1}-(-1)^{k-1} g_{j} d=f_{j-1}$ for any $j<n$. We write the defining equation for $g_{n}$ as

$$
g_{n} d=(-1)^{k-1}\left(d g_{n-1}-f_{n-1}\right)
$$

For simplicity we denote $\Theta_{i}=(-1)^{k-1}\left(d g_{n-1}-f_{n-1}\right)$. Thus $\Theta_{i}$ is defined in dimension $n-1$, and we have the following diagram:

$$
\begin{aligned}
& \left(A^{*}\right)_{n-1+k} \\
& \Theta_{i} \nearrow \\
& \left(A^{* \otimes i}\right)_{n-2} \quad \underset{d}{\rightarrow}\left(A^{* \otimes i}\right)_{n-1} \quad \vec{d} \quad\left(A^{* \otimes i}\right)_{n}
\end{aligned}
$$

Note that $\Theta_{i} d=0$; indeed

$$
\begin{aligned}
& (-1)^{k-1}\left\{d g_{n-1}-f_{n-1}\right\} d=(-1)^{k-1} d g_{n-1} d+d f_{n-2}= \\
& d\left\{(-1)^{k-1} g_{n-1} d+f_{n-2}\right\}=d d g_{n-2}=0 .
\end{aligned}
$$

We have used here the fact that $D f=0$, i.e. $\left.(-1)^{k} f_{n-1} d=d f_{n-2}\right)$. Since in the diagram above everything is functorial, we get

$$
\begin{array}{cccc} 
& \left.\hat{\Theta}_{i} \hat{A}^{*}\right)_{n-1+k} & \\
\left(\hat{A}^{* \otimes i}\right)_{n-2} \quad \underset{\hat{d}}{\rightarrow}\left(\hat{A}^{* \otimes i}\right)_{n-1} & \underset{\hat{h}}{\leftrightarrows} & \left(\hat{A}^{* \otimes i}\right)_{n}
\end{array}
$$

where $\hat{\Theta}_{i} \hat{d}=0$. Now we define $k_{i}=\hat{\Theta}_{i} \hat{h}^{i}$ and obtain

$$
k_{i} \hat{d}=\hat{\Theta}_{i} \hat{h}^{i} \hat{d}=\hat{\Theta}_{i}\left(1-\hat{d} \hat{h}^{i}\right)=\hat{\Theta}_{i}
$$

Next we define $g_{n}$ on $\left(A^{* \otimes i}(X)\right)_{n}$ by

$$
g_{n}=\Psi k_{i} \Phi
$$

and verify

$$
g_{n} d=\Psi k_{i} \Phi d=\Psi k_{i} \hat{d} \Phi=\Psi \hat{\Theta}_{i} \Phi=\Psi \Phi \Theta_{i}=\Theta_{i}
$$

as required. 


\section{Twisting cochains on $\operatorname{Hom}\left(\mathbf{B A}^{*}(X) \otimes \mathbf{B A}^{*}(X), \mathbf{A}^{*}(X)\right)$.}

For simplicity we will omit $X$ from the notations writing $A^{*}$ for $A^{*}(X)$.

Let us represent a homomorphism $E: B A^{*}(X) \otimes B A^{*}(X) \rightarrow A^{*}(X)$ by the collection of its components (restrictions on the tensor powers)

$$
\left\{E^{p, q}=\left.E\right|_{s^{-1} A^{\otimes p} \otimes s^{-1} A^{\otimes q}}\left(\left(s^{-1}\right)^{\otimes p} \otimes\left(s^{-1}\right)^{\otimes q}\right):\left(A^{*}\right)^{\otimes p} \otimes\left(A^{*}\right)^{\otimes q} \rightarrow A^{*}\right\}
$$

Note that if $\operatorname{deg} E=k$, then $\operatorname{deg} E^{p, q}=k-(p+q)$.

In the set of all twisting cochains from $B A^{*} \otimes B A^{*}$ to $A^{*}$ there exists one remarkable element. This is the "generator" $E_{s h}=p_{1} \mu_{s h}$ of the shuffle product $\mu_{s h}: B A^{*} \otimes B A^{*} \rightarrow B A^{*}$. Indeed, since the multiplication on $A^{*}$ is commutative, the Bar construction $\left(B A^{*}, d_{B A^{*}}, \mu_{s h}, \triangle\right)$ of $A^{*}$ is DGHA (see Subsection 1.10); i.e., $\mu_{s h} \in D C$, then by bijection 1.11 .1

$$
E_{s h}=p_{1} \mu_{s h}: B A^{*} \otimes B A^{*} \rightarrow A^{*}
$$

is a twisting cochain. It is well known that $E_{s h}$ consists of only two non trivial components: $E_{s h}^{0,1}=1: R \otimes A \rightarrow A$ and $E_{s h}^{0,1}=1: A \otimes R \rightarrow A$.

Let $F \in \operatorname{Hom}\left(B A^{*} \otimes B A^{*}, A^{*}\right)$. Let us denote by $F^{n}$ the sum of components $F^{p, q}$ of $F$ with $p+q=n$ :

$$
F^{n}=\sum_{p+q=n} F^{p, q}
$$

We call the number $p+q$ the filtration of $F^{p, q}$.

REMARK 55. It is easy to see that the product of two homogenous elements $F^{p_{1}, q_{1}}$ and $F^{p_{2}, q_{2}}$ in $\operatorname{Hom}\left(B A^{*} \otimes B A^{*}, A^{*}\right)$ is homogenous of the type $F^{p_{1}+p_{2}, q_{1}+q_{2}}$. Thus we see that the multiplication in $\mathrm{Hom}\left(B A^{*} \otimes B A^{*}, A^{*}\right)$ adds filtrations of the factors.

For simplicity we write $\mathcal{A}$ for $\operatorname{Hom}\left(B A^{*} \otimes B A^{*}, A^{*}\right)$; if $F \in \mathcal{A}$ has degree $m$ we write $F \in \mathcal{A}^{m}$. 
DeFinition 56. Let $n$ be a given number. We say that the elements $F_{1}, F_{2} \in$ $\mathcal{A}^{m}=\operatorname{Hom}^{m}\left(B A^{*} \otimes B A^{*}, A^{*}\right)$ coincide up to $n$ (write $\left.F_{1} \stackrel{n}{=} F_{2}\right)$ if

$$
F_{1}^{k}=F_{2}^{k}
$$

for any $k \leq n$.

Let $X$ be a simplicial set. Then the differential $D$ on $\operatorname{Hom}\left(B A^{*}(X) \otimes B A^{*}(X), A^{*}(X)\right)$ can be expressed as a sum

$$
D=D_{1}+D_{2}
$$

where

$$
D_{1} F=F\left(d_{\wedge} \otimes 1+1 \otimes d_{\wedge}\right) \quad D_{2} F=F\left(d_{\otimes} \otimes 1+1 \otimes d_{\otimes}\right)-(-1)^{|F|} d_{A^{*}(X)} F,
$$

$d_{\wedge}$ and $d_{\otimes}$ are given by the formula

$$
\begin{gathered}
d_{\wedge}\left[\Phi_{1}|\cdots| \Phi_{n}\right]=\sum_{i=0}^{n-2}\left[\bar{\Phi}_{1}|\cdots| \bar{\Phi}_{i}\left|\left(\bar{\Phi}_{i+1} \cdot a_{i+1}\right)\right| \Phi_{i+k+1}|\cdots| \Phi_{n}\right], \\
d_{\otimes}\left[\Phi_{1}|\cdots| \Phi_{n}\right]=\sum_{i=0}^{n-1}\left[\bar{\Phi}_{1}|\cdots| \bar{\Phi}_{i}\left|d \Phi_{i+1}\right| \Phi_{i+1}|\cdots| \Phi_{n}\right],
\end{gathered}
$$

where $\Phi_{i} \in A^{*}(X), \bar{\Phi}_{i}=(-1)^{\left|\Phi_{i}\right|+1} \cdot \Phi$ (see Subsection 1.10).

Proposition 57. Let $F$ be an element in $\mathcal{A}$ with $F^{n-1}=0$. Then equality $D F \stackrel{n}{=} 0$ guarantees that $D_{2} F^{n}=0$, i.e., $F^{n}$ is a chain map of (chain) complexes

$$
\sum_{p+q=n}\left(A^{*}(X) \otimes \cdots \stackrel{p}{\cdots} \otimes A^{*}(X)\right) \otimes\left(A^{*}(X) \otimes \cdot \stackrel{q}{*} \otimes A^{*}(X)\right) \rightarrow A^{*}(X) .
$$

Proof. Clear.

Now we are ready to prove the following lemma which is analogous to Theorem 21.

Lemma 58. Let $E, \bar{E} \in \mathcal{A}^{1}$ satisfy the following conditions

I) $D E=-E \cdot E$ and $D \bar{E}=-\bar{E} \cdot \bar{E}$ (i.e., $E$ are $\bar{E}$ twisting cochains);

II) $E^{1,0}=\bar{E}^{1,0}=1, E^{0,1}=\bar{E}^{0,1}=1$, and $E^{n, 0}=\bar{E}^{n, 0}=0, E^{0, n}=\bar{E}^{0, n}=0$ when $n \neq 1$. 
Then there exists $P \in \mathcal{A}^{0}, P^{0,0}=0$, satisfying

$$
\bar{E}=E+P \cdot E-\bar{E} \cdot P-D P .
$$

Proof. Note that it is enough to construct the sequence of elements in $\mathcal{A}^{0}$

$$
P_{(1)}, P_{(2)}, P_{(3)}, \cdots
$$

satisfying the following condition

I) $P_{(1)}=0$

II) $P_{(i)} \stackrel{i}{=} P_{(i+1)}, \quad i=1,2, \cdots$

III) $\left(P_{(i)}\right)^{k}=0 \quad$ when $k=0,1$ or $k>i$

IV) $D P_{(i)} \stackrel{i}{=} E-\bar{E}+P_{(i-1)} \cdot E-\bar{E} \cdot P_{(i-1)}$.

After constructing such a sequence we define $P$ by

$$
P \stackrel{i}{=} P_{(i)}, \quad i=1,2, \cdots
$$

Clearly, $P$ satisfies the required identity $\bar{E}=E+P \cdot E-\bar{E} \cdot P-D P$.

Assume that $P_{(1)}, P_{(2)}, \cdots, P_{(n-1)}$ have been already constructed. Now we must construct $P_{(n)}$ with the required conditions $I-I V$. Let us consider the following element in $\mathcal{A}^{1}$

$$
\beta(n-1)=E-\bar{E}+P_{(n-1)} \cdot E-\bar{E} \cdot P_{(n-1)}-D P_{(n-1)} .
$$

Conditions $I-I V$ and Remark 55 give

$$
\begin{aligned}
& \quad D \beta(n-1)= \\
& =D E-D \bar{E}+D P_{(n-1)} \cdot E+P_{(n-1)} \cdot D E-D \bar{E} \cdot P_{(n-1)}+\bar{E} \cdot D P_{(n-1)} \stackrel{n}{=} \\
& \stackrel{n}{=}-E \cdot E+\bar{E} \cdot \bar{E}+\left(E-\bar{E}+P_{(n-2)} \cdot E-\bar{E} \cdot P_{(n-2)}\right) \cdot E-P_{(n-1)} \cdot E \cdot E+ \\
& \quad \quad \quad \bar{E} \cdot \bar{E} \cdot P_{(n-1)}+\bar{E} \cdot\left(E-\bar{E}+P_{(n-2)} \cdot E-\bar{E} \cdot P_{(n-2)}\right)= \\
& \quad P_{(n-2)} \cdot E \cdot E-P_{(n-1)} \cdot E \cdot E+\bar{E} \cdot \bar{E} \cdot P_{(n-1)}-\bar{E} \cdot \bar{E} \cdot P_{(n-2)} \stackrel{n}{=} 0,
\end{aligned}
$$

where we used the fact that $P_{(n-2)} \cdot E \cdot E \stackrel{n}{=} P_{(n-1)} \cdot E \cdot E$; indeed, since $P^{(n-2)}, P^{(n-1)}$ and $E$ have no nontrivial components with filtration of 0 , multiplication adds filtrations 
(Remark 55) and $P^{(n-2)} \stackrel{n-2}{=} P^{(n-1)}$ (condition $\left.I I\right)$, then the elements $P^{(n-2)} \cdot E \cdot E$ and $P^{(n-1)} \cdot E \cdot E$ coincide up to $\left.n\right)$.

By definition, $\beta(n-1)$ has no nontrivial components with filtration $\leq n-1$; then by the Proposition 57

$$
D_{2} \beta(n-1)^{n}=0,
$$

or equivalently $\beta(n-1)^{n}$ is a cycle in

$$
\sum_{p+q=n}\left(A^{*}(X) \otimes \stackrel{p}{\cdots} \otimes A^{*}(X)\right) \otimes\left(A^{*}(X) \otimes \stackrel{q}{ } \otimes A^{*}(X)\right) \rightarrow A^{*}(X)
$$

Since the complex above is acyclic (see Lemma 53) there exists an element $P^{\prime}$ such that

$$
D_{2} P^{\prime}=\beta(n-1)^{n}
$$

Now we define $P_{(n)}$ by

$$
P_{(n)}=P_{(n-1)}+P^{\prime}
$$

Thus, it remains to show that $P_{(n)}$ together with $P_{(0)}, P_{(1)}, \cdots, P_{(n-1)}$ satisfies conditions $I-I V$. The first three conditions are immediate. Let us verify condition $I V$. Since $D_{2} P^{\prime}=\beta(n-1)^{n}$ and $\beta(n-1)$ has no nontrivial components with filtration $\leq n-1$, we have

$$
D P^{\prime} \stackrel{n}{=} \beta(n-1)
$$

then

$$
\begin{aligned}
& D P_{(n)}=D P_{(n-1)}+D P^{\prime} \stackrel{n}{=} D P_{(n-1)}+\beta(n-1)=D P_{(n-1)}-D P_{(n-1)}+ \\
& +E-\bar{E}+P_{(n-1)} \cdot E-\bar{E} \cdot P_{(n-1)}=E-\bar{E}+P_{(n-1)} \cdot E-\bar{E} \cdot P_{(n-1)} .
\end{aligned}
$$

Thus Lemma 58 is completely proved. 


\subsection{Multiplicativity up TO HOMOTOPY}

We complete this Section with the proof of Theorem 50 .

Proof of Theorem 50. Let us consider the following twisting cochain

$$
B A^{*}(X) \otimes B A^{*}(X) \stackrel{B\left\{\rho_{i}\right\} \otimes B\left\{\rho_{i}\right\}}{\longrightarrow} B C^{*}(X) \otimes B C^{*}(X) \stackrel{\mu_{E}}{\rightarrow} B C^{*}(X) \stackrel{P_{1}}{\rightarrow} C^{*}(X)
$$

where $\mu_{E}$ is a Baues multiplication from previous section (the entire composition is a twisting cochain being the composition of coalgebra maps and the twisting cochain $P_{1}$, see Subsection 1.9).

Since the first component $\rho=\rho_{1}: A^{*}(X) \rightarrow C^{*}(X)\left(<\rho \omega, c>=\int_{c} \omega\right)$ of Gugenheim's (DASH) map

$$
\rho_{i}: \otimes^{i} A^{*}(X) \rightarrow C^{*}(X) \quad(i \geq 1)
$$

induces an isomorphism in homology, we know from Theorem 20 that there exists twisting cochain

$$
\bar{E}: B A^{*}(X) \otimes B A^{*}(X) \rightarrow A^{*}(X)
$$

satisfying $P_{1} \mu_{E}\left(B\left\{\rho_{i}\right\} \otimes B\left\{\rho_{i}\right\}\right) \sim \sum_{i} \rho_{i}(\bar{E} \otimes \cdots \otimes \bar{E}) \triangle^{i}=P_{1} B\left\{\rho_{i}\right\} \mu_{\bar{E}}$, where

$$
\mu_{\bar{E}}: B A^{*}(X) \otimes B A^{*}(X) \rightarrow B A^{*}(X)
$$

is a coalgebra map induced by $\bar{E}$. We know that equivalent twisting cochains on $T(C, A)$ induce homotopic DG coalgebra maps on $\operatorname{Hom}(C, B A)$ (see Subsection 1.11); thus $\mu_{E}\left(B\left\{\rho_{i}\right\} \otimes B\left\{\rho_{i}\right\}\right) \sim B\left\{\rho_{i}\right\} \mu_{\bar{E}}$ in $D G C$, i.e.

$$
B\left\{\rho_{i}\right\}:\left(B A^{*}(X), d_{B A^{*}}, \triangle, \mu_{\bar{E}}\right) \rightarrow\left(B C^{*}(X), d_{B C^{*}}, \triangle, \mu_{E}\right)
$$

is multiplicative up to homotopy, but the randomly chosen $\mu_{\bar{E}}$ may not be $\mu_{s h}$.

Let us assume that $\bar{E}^{0,1}=1, \bar{E}^{1,0}=1$ and $\bar{E}^{0, n}=0, \bar{E}^{n, 0}=0$ when $n \neq 1$ (we will show below that we can achieve this). Then we have two twisting cochains $E_{s h}\left(E_{s h^{-}}\right.$the generator of shuffle product $\left.\mu_{s h}\right)$ and $\bar{E}$ on $\operatorname{Hom}\left(B A^{*} \otimes B A^{*}, A^{*}\right)$ 
satisfying the conditions of Lemma 58; therefore there exists a homomorphism $P \in$ $\operatorname{Hom}\left(B A^{*}(X) \otimes B A^{*}(X), A^{*}(X)\right)$ such that $E_{s h} \stackrel{P}{\sim} \bar{E}$, hence $\mu_{s h} \sim \mu_{\bar{E}}$, or equivalently

$$
1:\left(B A^{*}(X), d_{B A^{*}}, \triangle, \mu_{s h}\right) \rightarrow\left(B A^{*}(X), d_{B A^{*}}, \triangle, \mu_{\bar{E}}\right)
$$

is multiplicative up to homotopy. Then the composition

$$
\begin{gathered}
B\left\{\rho_{i}\right\} \cdot 1:\left(B A^{*}(X), d_{B A^{*}}, \triangle, \mu_{s h}\right) \rightarrow\left(B A^{*}(X), d_{B A^{*}}, \triangle, \mu_{\bar{E}}\right) \\
\rightarrow\left(B C^{*}(X), d_{B C^{*}}, \triangle, \mu_{E}\right)
\end{gathered}
$$

produces the homotopy multiplicative Gugenheim map

$$
B\left\{\rho_{i}\right\}:\left(B A^{*}(X), d_{B A^{*}}, \triangle, \mu_{s h}\right) \rightarrow\left(B C^{*}(X), d_{B C^{*}}, \triangle, \mu_{E}\right)
$$

Thus, it remains to show that $\bar{E}^{0,1}=1, \bar{E}^{1,0}=1$ and $\bar{E}^{0, n}=0, \bar{E}^{n, 0}=0$ when $n \neq 1$. First of all, note that $\mu_{\bar{E}}$ is homotopy commutative, homotopy associative and has a homotopy unit. Let us verify the first assertion, exactly the same argument can be applied to show the rest. Indeed,

$$
\begin{gathered}
\left\{\rho_{i}\right\}_{*}(\bar{E})=P_{1} B\left\{\rho_{i}\right\} \mu_{\bar{E}} \sim P_{1} \mu_{E}\left(B\left\{\rho_{i}\right\} \otimes B\left\{\rho_{i}\right\}\right) \sim P_{1} \mu_{E} T\left(B\left\{\rho_{i}\right\} \otimes B\left\{\rho_{i}\right\}\right)= \\
=P_{1} \mu_{E}\left(B\left\{\rho_{i}\right\} \otimes B\left\{\rho_{i}\right\}\right) T \sim P_{1} B\left\{\rho_{i}\right\} \mu_{\bar{E}} T=P_{1} B\left\{\rho_{i}\right\} \mu_{\bar{E} T}=\left\{\rho_{i}\right\}_{*}(\bar{E} T),
\end{gathered}
$$

where we used homotopy multiplicativity of the Baues product, $\mu_{E} \sim \mu_{E} T$. Since $\left\{\rho_{i}\right\}_{*}(\bar{E}) \sim\left\{\rho_{i}\right\}_{*}(\bar{E} T)$, according to the bijection of Theorem 20 the twisting cochains $\bar{E}$ and $\bar{E} T$ are equivalent, hence they induce homotopic coalgebra maps, $\mu_{\bar{E}} \sim \mu_{\bar{E}} T$; i.e. $\mu_{\bar{E}}$ is homotopy commutative. Now by Proposition $37 \mu_{\bar{E}}$ can be replaced by the new product

$$
\mu_{\tilde{E}} \sim \mu_{\bar{E}}: B A^{*} \otimes B A^{*} \rightarrow B A^{*}
$$

which has a strict unit. The latter implies that the twisting cochain

$$
\tilde{E}=P_{1} \mu_{\tilde{E}}: B A^{*}(X) \otimes B A^{*}(X) \rightarrow B A^{*}(X) \rightarrow A^{*}(X)
$$

has $\tilde{E}^{0,1}=1, \tilde{E}^{1,0}=1$ and $\tilde{E}^{0, n}=0, \tilde{E}^{n, 0}=0$ when $n \neq 1$. This completes the proof. 


\section{3. $\tilde{B}_{\infty}$-ALGEBRA CATEGORY}

\section{$3.1 B_{\infty}$-ALGEBRA}

In this section we recall the notion of a $B_{\infty}$-algebra. We show that the singular cochain complex $C^{*}(X, R)$ endowed with the Baues operations from previous sections is a special case of a $B_{\infty}$-algebra; see also Baues' comment on this fact in [7].

The notion of $B_{\infty}$-algebra was introduced by Getzler and Jones in [19] as an additional structure on a chain complex $H$ which turns the bar-construction $B H$ into a $D G$-Hopf algebra. So this structure requires a new differential (which should be a coderivation with respect to standard coprodact on $B H) d_{m}: B H \rightarrow B H$ and a new associative product $\mu: B H \otimes B H \rightarrow B H$ so that $d_{m}: B H \rightarrow B H$ is a derivation.

Having in mind the structure of a differential on $B H$ (Subsection 1.10) and of a product $B H \otimes B H \rightarrow B H$ (Subsection 1.14) we can formulate the definition of a $B_{\infty}$-algebra as follows

Definition 59. A $B_{\infty}$-algebra is defined as an $A_{\infty}$-algebra $\left(H,\left\{m_{i}\right\}\right)$ together with a set of operations $E=\left\{E_{p, q}:\left(\otimes^{p} H\right) \otimes\left(\otimes^{q} H\right) \rightarrow H\right\}$, such that $E_{0,1}=1$, $E_{1,0}=1, E_{0, n}=E_{n, 0}=0 n \neq 1$ and

$$
\begin{aligned}
& \text { (i) } \sum_{j=0}^{p-i} \sum_{i=1}^{p} E_{p-i+1, q}\left(a_{1}, \cdots, a_{j}, m_{i}\left(a_{j+1}, \cdots, a_{i+j}\right), a_{i+j+1}, \cdots, a_{p} \mid b_{1}, \cdots, b_{q}\right)+ \\
& +\sum_{l=0}^{q-k} \sum_{k=1}^{q} E_{p, q-k-1}\left(a_{1}, \cdots, a_{p} \mid b_{1}, \cdots, b_{l}, m_{k}\left(b_{l+1}, \cdots, b_{k+l}\right), b_{k+l+1}, \cdots, b_{q}\right)=
\end{aligned}
$$




$$
\begin{aligned}
& =\sum \sum_{t=1}^{p+q} m_{t}\left(E_{p_{1}, q_{1}}\left(a_{1}, \cdots, a_{p_{1}} \mid b_{1}, \cdots, b_{q_{1}}\right), \cdots\right. \\
& p_{1}+\ldots+p_{t}=p \\
& q_{1}+\ldots+q_{t}=q \\
& \cdots, E_{p_{t}, q_{t}}\left(a_{p-p_{t}+1}, \cdots, a_{p} \mid b_{q-q_{t}+1}, \cdots, b_{q}\right) \text {; } \\
& \sum \sum_{t=1}^{p+q} E_{t, g}\left(E_{p_{1}, q_{1}}\left(a_{1}, \cdots, a_{p_{1}} \mid b_{1}, \cdots, b_{q_{1}}\right), \cdots\right. \\
& p_{1}+\ldots+p_{t}=p \\
& q_{1}+\ldots+q_{t}=q \\
& \left.\cdots, E_{p_{t}, q_{t}}\left(a_{p-p_{t}+1}, \cdots, a_{p} \mid b_{q-q_{t}+1}, \cdots, b_{q}\right) \mid c_{1}, \cdots, c_{g}\right)= \\
& \sum \sum_{k=1}^{q+g} E_{p, k}\left(a_{1}, \cdots, a_{p} \mid E_{r_{1}, g_{1}}\left(b_{1}, \cdots, b_{r_{1}} \mid c_{1}, \cdots, c_{g_{1}}\right), \cdots\right. \\
& r_{1}+\ldots+r_{k}=q \\
& g_{1}+\ldots+g_{k}=g \\
& \left.\cdots, E_{r_{k}, g_{k}}\left(b_{q-r_{k}+1}, \cdots, b_{q} \mid c_{g-g_{k}+1}, \cdots, c_{g}\right)\right) .
\end{aligned}
$$

It follows from (i) that the map $E:\left(B H \otimes B H, d_{m \otimes m}, \Delta_{\otimes}\right) \rightarrow\left(H,\left\{m_{i}\right\}\right)$, given by $\left.E\right|_{\left(\otimes^{p} H\right) \otimes\left(\otimes^{q} H\right)}=E_{p, q}$, is an $A_{\infty}$-twisting cochain. Thus it generates a DG-coalgebra map $\mu_{E}:\left(B H \otimes B H, d_{m \otimes m}, \Delta_{\otimes}\right) \rightarrow\left(B H, d_{m}, \Delta\right)$, defined by

$$
\begin{aligned}
& \mu_{E}\left(a_{1}, \cdots, a_{p} \mid b_{1}, \cdots, b_{q}\right)= \\
& =\sum_{\substack{p_{1}+\ldots+p_{t}=p \\
q_{1}+\ldots+q_{t}=q}}^{p+q} \sum_{t=1}^{p+q} E_{p_{1}, q_{1}}\left(a_{1}, \cdots, a_{p_{1}} \mid b_{1}, \cdots, b_{q_{1}}\right), \cdots \\
& \cdots, E_{p_{t}, q_{t}}\left(a_{p-p_{t}+1}, \cdots, a_{p} \mid b_{q-q_{t}+1}, \cdots, b_{q}\right) .
\end{aligned}
$$

Condition (ii) guarantees that the product defined above is associative; so the Barconstruction $\left(B H, d_{m}, \Delta, \mu_{E}\right)$ of a $B_{\infty}$-algebra $\left(H,\left\{m_{i}\right\},\left\{E_{p, q}\right\}\right)$ is $D G$-Hopf algebra.

Note that a Homotopy G-algebra $\left(A, d, \mu,\left\{E_{1, q}\right\}\right)$ can be considered as a $B_{\infty^{-}}$ $\operatorname{algebra}\left(A,\left\{m_{i}\right\},\left\{E_{p, q}\right\}\right)$ with $m_{3}=m_{4}=\cdots=0$, and $E_{p, q}=0$ unless $p=1$ and $E_{0,1}=1$. In such a case conditions $(i)-(i i)$ are equivalent to the conditions 
$(i)-($ iii $)$ of the Definition 46. Since we know that $\left(C^{*}(X, R), d_{C^{*}(X, R)}, \smile\right)$ endowed with Baues operations $\left\{E_{1, k}\right\}$ carries the structure of a Homotopy G-Algebra then from the discussion above it is obvious that $\left(C^{*}(X, R), d_{C^{*}(X, R)}, \smile,\left\{E_{1, k}\right\}\right)$ is a $B_{\infty^{-}}$ algebra.

Note that any commutative $A_{\infty}$-algebra $\left(A,\left\{m_{i}\right\}\right)$ also can be regarded as a $B_{\infty^{-}}$ algebra of the following type $\left(A,\left\{m_{i}\right\},\left\{E_{1,0}=E_{0,1}=1, E_{p, q}=0\right.\right.$ otherwise $\left.\}\right)$; the first condition in the definition above simply reduces to $m_{k} s_{i, k-i}=0$.

DeFinition 60. We define a $B_{\infty}$-algebra morphism

$$
f:\left(A,\left\{m_{i}^{A}\right\},\left\{E_{p, q}^{A}\right\}\right) \rightarrow\left(B,\left\{m_{i}^{B}\right\},\left\{E_{p, q}^{B}\right\}\right)
$$

as an $A_{\infty}$-algebra map

$$
f:\left(A,\left\{m_{i}^{A}\right\}\right) \rightarrow\left(B,\left\{m_{i}^{B}\right\}\right)
$$

such that the coalgebra map

$$
B f:\left(B A, B d_{m^{A}}, \mu_{E^{A}}, \Delta\right) \rightarrow\left(B B, B d_{m^{B}}, \mu_{E^{B}}, \Delta\right)
$$

of appropriate bar constructions is multiplicative up to homotopy, i.e. there exists a coderivation homotopy from $B f \mu_{E^{A}}$ to $\mu_{E^{B}}(B f \otimes B f)$.

A $B_{\infty}$-algebra morphism

$$
f:\left(A,\left\{m_{i}^{A}\right\},\left\{E_{p, q}^{A}\right\}\right) \rightarrow\left(B,\left\{m_{i}^{B}\right\},\left\{E_{p, q}^{B}\right\}\right)
$$

is called quasi-isomorphism, isomorphism, etc. if $B f$ is so in $D G C$.

Definition 61. Two $B_{\infty}$-algebra morphisms

$$
f, g:\left(A,\left\{m_{i}^{A}\right\},\left\{E_{p, q}^{A}\right\}\right) \rightarrow\left(B,\left\{m_{i}^{B}\right\},\left\{E_{p, q}^{B}\right\}\right)
$$

are called homotopic, $f \sim g \in B_{\infty}$-Alg, if $B f \sim B g \in D G C$.

It is immediate that $\sim$ is an equivalence relation. 
Definition 62. $B_{\infty}$-algebras $\left(A,\left\{m_{i}^{A}\right\},\left\{E_{p, q}^{A}\right\}\right)$ and $\left(B,\left\{m_{i}^{B}\right\},\left\{E_{p, q}^{B}\right\}\right)$ we call weakly equivalent if they are connected by the chain

$$
(A, d) \stackrel{\simeq}{\longleftarrow}(Z(0), d) \stackrel{\simeq}{\longrightarrow}(Z(1), d) \stackrel{\simeq}{\longleftarrow} \ldots \stackrel{\simeq}{\longleftarrow}(Z(k), d) \stackrel{\simeq}{\longrightarrow}(B, d)
$$

of quasi-isomorphisms of $B_{\infty}$-algebras.

It is easy to check that $B_{\infty}$-algebras and their morphisms form a category which we denote by $B_{\infty}-A l g$.

We know that the Bar construction $\left(B C^{*}(X, Q), d_{B C^{*} X}, \Delta, \mu_{E}\right)$ of a 1-reduced simplicial set $X$ is a $D G$-Hopf algebra. Moreover we know that the product $\mu_{E}$ is homotopy commutative so that $\left(B C^{*}(X, Q), d_{B C^{*} X}, \Delta, \mu_{E}\right) \in H A H$. In the upcoming sections we essentially need homotopy commutative products which encourages us to slightly modify the notion of $B_{\infty}$-algebra.

Definition 63. An $\left(H,\left\{m_{i}\right\},\left\{E_{p, q}\right\}\right)$ is called a $\tilde{B}_{\infty}$-algebra if the Bar construction $\left(B H, d_{m}, \Delta, \mu_{E}\right) \in H A H$.

Note that we do not require in the definition above strict associativity of the multiplication $\mu_{E}$, as it was the case for $B_{\infty}$-algebras. Definition 63 yields to up to homotopy commutative and up to homotopy associative multiplication.

Let $X$ be a 1-reduced simplicial set. In Subsection 1.14 we constructed Baues operations $\left\{E_{1, k}\right\}$ and $\left\{F_{p, q}\right\}$ on $C^{*}(X, R)$. We will show below that

$$
\left(C^{*}(X, R), d_{C^{*}(X, R)}, \smile,\left\{E_{1, k}\right\},\left\{F_{p, q}\right\}\right)
$$

is an object in $\tilde{B}_{\infty}$-Alg. Indeed we know that operations $\left\{E_{1, k}\right\}$ define a strictly associative multiplication $\mu_{E}$ (which also has a strict unit) on the Bar construction $B C^{*}(X, R)$. Moreover, operations $\left\{F_{p, q}\right\}$ uniquely define a coderivation homotopy $D_{F}$ from $\mu_{E}$ to $\mu_{E} T$ making the product $\mu_{E}$ homotopy commutative; i.e. the Bar construction $\left(B C^{*}(X, R), d_{C^{*}(X, R)}, \Delta, \mu_{E}\right) \in H A H$. Then by Definition 63 we have a $\tilde{B}_{\infty^{-}}$algebra structure on $\left(C^{*}(X, R), d_{C^{*}(X, R)}, \smile,\left\{E_{1, k}\right\},\left\{F_{p, q}\right\}\right)$. 
DeFinition 64. We define a $\tilde{B}_{\infty}$-algebra morphism

$$
f:\left(A,\left\{m_{i}^{A}\right\},\left\{E_{p, q}^{A}\right\}\right) \rightarrow\left(B,\left\{m_{i}^{B}\right\},\left\{E_{p, q}^{B}\right\}\right)
$$

as an $A_{\infty}$-algebra map

$$
f:\left(A,\left\{m_{i}^{A}\right\}\right) \rightarrow\left(B,\left\{m_{i}^{B}\right\}\right)
$$

such that the coalgebra map

$$
B f:\left(B A, B d_{m^{A}}, \mu_{E^{A}}, \Delta\right) \rightarrow\left(B B, B d_{m^{B}}, \mu_{E^{B}}, \Delta\right)
$$

of appropriate bar constructions is multiplicative up to homotopy, i.e. there exists a coderivation homotopy from $B f \mu_{E^{A}}$ to $\mu_{E^{B}}(B f \otimes B f)$.

A $\tilde{B}_{\infty}$-algebra morphism

$$
f:\left(A,\left\{m_{i}^{A}\right\},\left\{E_{p, q}^{A}\right\}\right) \rightarrow\left(B,\left\{m_{i}^{B}\right\},\left\{E_{p, q}^{B}\right\}\right)
$$

is called a quasi-isomorphism, isomorphism, etc. if $B f$ is so in $H A H$.

Definition 65. Two $\tilde{B}_{\infty}$-algebra morphisms

$$
f, g:\left(A,\left\{m_{i}^{A}\right\},\left\{E_{p, q}^{A}\right\}\right) \rightarrow\left(B,\left\{m_{i}^{B}\right\},\left\{E_{p, q}^{B}\right\}\right)
$$

are called homotopic, $f \sim g \in \tilde{B}_{\infty}-A l g$, if $B f \sim B g \in H A H$, i.e. there is a coderivation homotopy from $B f$ to $B g$.

It is immediate that $\sim$ is an equivalence relation.

Proposition 66. Let $f, g:\left(A,\left\{m_{i}^{A}\right\},\left\{E_{p, q}^{A}\right\}\right) \rightarrow\left(B,\left\{m_{i}^{B}\right\},\left\{E_{p, q}^{B}\right\}\right)$ be $\tilde{B}_{\infty}$-algebra morphisms. Then $f \sim g \in \tilde{B}_{\infty}$-Alg if and only if $B f \sim B g \in H A H$.

Proof. Directly follows from the Definitions 64 and 65 .

Definition 67. $\tilde{B}_{\infty}$-algebras $\left(A,\left\{m_{i}^{A}\right\},\left\{E_{p, q}^{A}\right\}\right)$ and $\left(B,\left\{m_{i}^{B}\right\},\left\{E_{p, q}^{B}\right\}\right)$ are called weakly equivalent if they are connected by the chain

$$
(A, d) \stackrel{\simeq}{\longleftarrow}(Z(0), d) \stackrel{\simeq}{\longrightarrow}(Z(1), d) \stackrel{\simeq}{\longleftarrow} \cdots \stackrel{\simeq}{\longleftarrow}(Z(k), d) \stackrel{\simeq}{\longrightarrow}(B, d)
$$

of quasi-isomorphisms of $\tilde{B}_{\infty}$-algebras. 
It is easy to check that $\tilde{B}_{\infty}$-algebras and their morphisms form a category which we denote by $\tilde{B}_{\infty}-A l g$.

Assigning to each 1-reduced simplicial set $X$ an appropriate $\tilde{B}_{\infty}$-Algebra

$$
X \mapsto\left(C^{*}(X), d_{C^{*}(X)}, \smile,\left\{E_{1, k}\right\},\left\{F_{p, q}\right\}\right)
$$

defines a correspondence from 1-reduced simplicial sets to 1 -connected $\tilde{B}_{\infty}$-algebras. To show that this correspondence is natural we need to show that any simplicial map $f: X \rightarrow Y$ induces a $\tilde{B}_{\infty}$-Algebra morphism

$$
C^{*}(f):\left(C^{*}(Y), d_{C^{*}(Y)}, \smile,\left\{E_{1, k}^{Y}\right\},\left\{F_{p, q}^{Y}\right\}\right) \rightarrow\left(C^{*}(X), d_{C^{*}(X)}, \smile,\left\{E_{1, k}^{X}\right\},\left\{F_{p, q}^{X}\right\}\right)
$$

By Definition 64 we need to show that the coalgebra map

$$
B C^{*}(f):\left(B C^{*}(Y), d_{B C^{*}(Y)}, \Delta, \mu_{E^{Y}}\right) \rightarrow\left(B C^{*}(X), d_{B C^{*}(X)}, \Delta, \mu_{E^{X}}\right)
$$

is multiplicative up to homotopy, i.e. $B C^{*}(f) \mu_{E^{Y}} \sim \mu_{E^{X}}\left(B C^{*}(f) \otimes B C^{*}(f)\right)$. But $B C^{*}(f)$ is strictly multiplicative not just homotopy multiplicative [7]; it is straightforward to check that the singular cochain map $C^{*}(f): C^{*}(Y) \rightarrow C^{*}(X)$ commutes with operations $\left\{E_{1, k}\right\}$ which is a necessary and sufficient condition for multiplicativity of $B C^{*}(f)$.

We state below some key results of Baues [7], which we will use in the next section.

THEOREM 68. Let $X$ be a 1-reduced simplicial set. Then there is a product $\mu_{\tilde{E}}$ on the bar construction $B C^{*}(X, Q)$ such that $\left(B C^{*}(X, Q), \mu_{\tilde{E}}\right)$ is a commutative differential Hopf algebra. Moreover there is a derivation homotopy $F: \mu_{E} \simeq \mu_{\tilde{E}}$. Both $\mu_{\tilde{E}}$ and $F$ are natural for $X \in s_{S e t_{1}}$ so that one gets a commutative diagram of functors

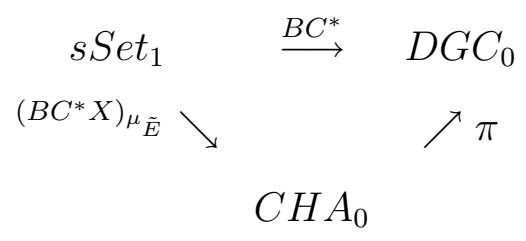

where sSet and $_{\mathrm{CH}} \mathrm{A}_{0}$ are categories of 1-reduced simplicial sets and connected commutative Hopf algebras respectively. 
TheOrem 69. Let $X$ be a 1-reduced simplicial set. Then there is a differential $d=d_{\mathcal{L}^{c}}$ on the free Lie coalgebra $\mathcal{L}^{c}\left(s^{-1} I C^{*}(X, Q)\right)$ such that $\mathcal{L}^{c}(X, Q)=$ $\left(\mathcal{L}^{c}\left(s^{-1} I C^{*}(X, Q)\right), d_{\mathcal{L}^{c}}\right)$ defines a functor

$$
\mathcal{L}^{c}: s S e t \longrightarrow D L C_{1}
$$

Here $\mathcal{L}^{c}$ carries a map $f: X \longrightarrow Y$ to the induced map $\mathcal{L}^{c}(f)=\mathcal{L}^{c}\left(s^{-1} I C^{*} f\right)$ which is a differential map. Moreover there is an isomorphism of commutative differential Hopf algebras

$$
\beta:\left(U^{c} \mathcal{L}^{c}(X, Q), \mu_{U^{c} \mathcal{L}^{c}(X)}\right) \longrightarrow\left(B C^{*}(X, Q), \mu_{\tilde{E}}\right)
$$

Here $U^{c} \mathcal{L}^{c}(X, Q)=\left(U^{c} \mathcal{L}^{c}\left(s^{-1} I C^{*}(X, Q)\right), d\right)$ is the universal coenveloping coalgebra of the Lie coalgebra $\mathcal{L}^{c}\left(s^{-1} I C^{*}(X, Q)\right)$ and $\mu_{\tilde{E}}$ is the product on the bar construction from the theorem above. The isomorphism $\beta$ is natural in $X \in$ sSet $_{1}$ and induces the identity on $s^{-1} I C^{*}(X, Q)$.

It is worth mentioning that the differential $d_{\mathcal{L}^{c}}$ on $\mathcal{L}^{c}\left(s^{-1} I C^{*}(X, Q)\right)$ was defined by $d_{\mathcal{L}^{c}}=\beta^{-1} d_{B C^{*} X} \beta$. Consequently we have three naturally isomorphic differential Hopf algebras

$$
\begin{aligned}
\left(B C^{*}(X, Q), d_{B C^{*} X}, \mu_{\tilde{E}}\right) & =\left(U^{c} \mathcal{L}^{c}(X, Q), U^{c} d_{\mathcal{L}^{c}}, \mu_{U^{c} \mathcal{L}^{c}(X)}\right) \\
& =\left(B C^{*}(X, Q), U^{c} d_{\mathcal{L}^{c}}, \mu_{s h}\right)
\end{aligned}
$$

(see [7], or Subsection 1.9 above).

We know that the differential $U^{c} d_{\mathcal{L}^{c}}$ on $\left(B C^{*}(X, Q), \mu_{s h}, U^{c} d_{\mathcal{L}^{c}}\right)$ canonically defines a commutative $A_{\infty}$-algebra structure $\left\{m_{i}:\left(C^{*}(X, Q)^{\otimes i} \rightarrow C^{*}(X, Q)\right\}\right.$ on $C^{*}(X, Q)$ (see Definition 14). Baues computed $m_{1}$ and $m_{2}$ explicitly.

TheOrem 70. Let $X$ be a 1-reduced simplicial set. Then the first two components of the differential $U^{c} d_{\mathcal{L}^{c}}$ are given by

$$
m_{1}=d_{C^{*}(X, Q)}
$$


and

$$
m_{2}=\frac{1}{2}(\smile+\smile T)
$$

where $\smile$ is an Alexander-Whitney multiplication on the singular cochain complex $C^{*}(X, Q)$.

Clearly $m_{2}$ is commutative, i.e. $m_{2} s_{1,1}=0$. We finish this section with the following

REMARK 71. The functor $\mathcal{L}^{c}$ in Theorem 69 induces an equivalence of rational homotopy categories

$$
\mathcal{H}_{o} \mathcal{L}^{c}: \mathcal{H o s S e t}_{1} \rightarrow \mathcal{H} o D L C_{1}
$$

where $\mathcal{H}$ o denotes a localization with respect to rational cohomology isomorphism, see $[7]$.

\section{$3.2 A_{\infty}$-Algebra struCture IN HOMOLOGY}

Let $X$ be a 1-reduced simplicial set and $\left(A^{*}(X, Q), d_{A^{*}(X)}, \wedge\right)$ be a Sullivan $D G$ algebra of polynomial differential forms on $X$, see Section 2 . We mentioned above that in homology $H\left(A^{*}(X, Q), d_{A^{*}(X)}, \wedge\right)$ there is a commutative $A_{\infty}$-algebra structure

denoted by $\left(H A^{*}(X, Q),\left\{m_{i}^{H A^{*}(X)}\right\}\right)$; the commutativity of Sullivan's cochains was strongly applied in the construction.

On the other hand, although the $D G$-algebra $\left(C^{*}(X, Q), d_{C^{*}(X)}, \smile\right)$ of the singular cochains almost never is commutative, the Baues' higher homotopies still allow us to construct a commutative $A_{\infty}$-algebra structure on $H\left(C^{*}(X, Q)\right)$. Indeed, from the preceding section we know that there is a commutative $A_{\infty}$-algebra structure on $C^{*}(X, Q)$ with $m_{1}=d_{C^{*} X}$ and $m_{2}=\frac{1}{2}(\smile+\smile T)$; then by Theorem 24 we have the following 
Proposition 72. There exist a minimal commutative $A_{\infty}$-algebra structure on $H C^{*}(X, Q)$ and a quasi-isomorphism of commutative $A_{\infty}$-algebras

$$
\left.\left\{f_{i}^{\prime}\right\}:\left(H C^{*}(X, Q)\right),\left\{m_{i}^{H C^{*}(X)}\right\}\right) \rightarrow\left(C^{*}(X, Q),\left\{m_{i}\right\}\right)
$$

Thus for a topological space $X$ we have now two minimal commutative $A_{\infty^{-}}$ algebras on homology $H(X, Q)$; one is obtained through Sullivan's commutative DG-algebra $A^{*}(X, Q)$ and its appropriate $A_{\infty}$-algebra is denoted by $\left(H A^{*}(X, Q)=\right.$ $\left.H(X, Q),\left\{m_{i}^{H A^{*}(X)}\right\}\right)$, and the second is obtained through the singular cochain algebra $C^{*}(X, Q)$ and its appropriate $A_{\infty}$-algebra is denoted by $\left(H(X, Q),\left\{m_{i}^{H C^{*}(X)}\right\}\right)$. We show below that they are isomorphic in $C$ - $A_{\infty}$-Alg category.

THEOREM 73. There is a commutative $A_{\infty}$-algebra isomorphism

$$
\left.P_{1} \Sigma:\left(H A^{*}(X, Q),\left\{m_{i}^{H A^{*}(X)}\right\}\right) \rightarrow\left(H C^{*}(X, Q)\right),\left\{m_{i}^{H C^{*}(X)}\right\}\right) .
$$

Proof. Let us consider the following composition of the bar construction maps

$$
\begin{aligned}
& \left(B H A^{*}(X, Q), d_{m^{H A^{*}(X)}}, \Delta, \mu_{s h}\right) \quad\left(B H C^{*}(X, Q), d_{m^{H C^{*}(X)}}, \Delta, \mu_{s h}\right) \\
& \downarrow B f \quad \uparrow B g^{\prime} \\
& \left(B A^{*}(X, Q), d_{B A^{*}(X)}, \Delta, \mu_{s h}\right) \quad\left(B C^{*}(X, Q), d_{m^{C^{*}(X)}}, \Delta, \mu_{s h}\right) \\
& \downarrow B \rho \quad \uparrow \beta \\
& \left(B C^{*}(X, Q), d_{B C^{*}(X)}, \Delta, \mu_{E}\right) \quad \stackrel{1}{\rightarrow} \quad\left(B C^{*}(X, Q), d_{B C^{*}(X)}, \Delta, \mu_{\tilde{E}}\right)
\end{aligned}
$$

Here $B f$ is multiplicative and $B g^{\prime}$ is multiplicative up to homotopy (see Theorem 24); also $\beta$ is the Hopf algebra map of Theorem 69 and $B \rho$ is multiplicative up to homotopy by Theorem 50 . Therefore the entire composition

$$
\Gamma:\left(B H A^{*}(X, Q), d_{m^{H A^{*}(X)}}, \Delta, \mu_{s h}\right) \rightarrow\left(B H C^{*}(X), d_{m^{H C^{*}(X, Q)}}, \Delta, \mu_{s h}\right)
$$

is multiplicative up to homotopy as well. Moreover each of the maps is a quasiisomorphism, and so is $\Gamma$; but the quasi-isomorphism of the bar constructions over minimal $A_{\infty}$-algebras is an isomorphism; i.e. $\Gamma$ is an isomorphism in $H A H$. Then by 
Proposition 48 there exists $D G$-Hopf algebra isomorphism

$$
\Sigma:\left(B H A^{*}(X, Q), d_{m^{H A^{*}(X)}}, \Delta, \mu_{s h}\right) \rightarrow\left(B H C^{*}(X, Q), d_{m^{H C^{*}(X)}}, \Delta, \mu_{s h}\right)
$$

such that $\Sigma \sim \Gamma$ in $H A H$. This implies that the underlying commutative $A_{\infty}$-algebra morphism

$$
P_{1} \Sigma:\left(H A^{*}(X, Q),\left\{m_{i}^{H A^{*}(X)}\right\}\right) \rightarrow\left(H C^{*}(X, Q),\left\{m_{i}^{H C^{*}(X)}\right\}\right)
$$

is both commutative and an isomorphism since $\Sigma$ is (Subsection 1.10). 


\section{Rational homotopy tYPe}

By a space below we mean a CW complex; we call a CW complex $X$ 1-reduced if its 1-skeleton $X^{1}$ is trivial. For simplicity we suppress $Q$ from the notation, just writing $C^{*}(X), H A H$, for $C^{*}(X, Q), H A H_{1}(Q)$ respectively.

TheOrem 74. The 1-reduced $X$ and $Y$ have the same rational homotopy type if and only if $\left(C^{*}(X), d_{C^{*}(X)}, \smile,\left\{E_{1, k}^{X}\right\},\left\{F_{p, q}^{X}\right\}\right)$ and $\left(C^{*}(Y), d_{C^{*}(Y)}, \smile,\left\{E_{1, k}^{Y}\right\},\left\{F_{p, q}^{Y}\right\}\right)$ are weakly equivalent in the $\tilde{B}_{\infty}$-algebra category.

Proof. Let us assume that $X$ and $Y$ have the same rational homotopy type; this implies that there exists a chain

$$
X=T_{0} \stackrel{f_{1}}{\longrightarrow} T_{1} \stackrel{f_{2}}{\longleftarrow} T_{2} \rightarrow \cdots \stackrel{f_{n+1}}{\longleftarrow} T_{n+1}=Y
$$

of 1-reduced spaces and rational homotopy equivalences, i.e. continuous maps inducing isomorphism on the rational cohomology ring. For simplicity let us assume that there is short such chain:

$$
X \stackrel{f_{1}}{\longrightarrow} T \stackrel{f_{2}}{\longleftarrow} Y
$$

the long version is proved exactly by same argument. From Section 3 we know that

each of the maps, $f_{1}$ and $f_{2}$, induces $\tilde{B}_{\infty}$-algebra morphisms $C^{*} f_{1}$ and $C^{*} f_{2}$. So we have a chain of $\tilde{B}_{\infty}$-algebras and $\tilde{B}_{\infty}$-algebra morphisms

$$
\begin{gathered}
\left(C^{*}(X), d_{C^{*}(X)}, \smile,\left\{E_{1, k}^{X}\right\},\left\{F_{p, q}^{X}\right\}\right) \stackrel{C^{*}\left(f_{1}\right)}{\longleftarrow}\left(C^{*}(T), d_{C^{*}(T)}, \smile,\left\{E_{1, k}^{T}\right\},\left\{F_{p, q}^{T}\right\}\right) \stackrel{C^{*}\left(f_{2}\right)}{\longrightarrow} \\
\longrightarrow\left(C^{*}(Y), d_{C^{*}(Y)}, \smile,\left\{E_{1, k}^{Y}\right\},\left\{F_{p, q}^{Y}\right\}\right)
\end{gathered}
$$

So it remains to show that each of the maps, $C^{*} f_{1}$ and $C^{*} f_{2}$, is a quasi-isomorphism in $\tilde{B}_{\infty}-A l g$. By the definition of quasi-isomorphism in $\tilde{B}_{\infty}-A l g$ we need to show 
that the Bar construction maps $B C^{*} f_{1}$ and $B C^{*} f_{2}$ are quasi-isomorphisms. But they are, since both $C^{*} f_{1}$ and $C^{*} f_{2}$ are quasi-isomorphisms in $D G C$ (see Subsection 1.10, Definition 13). Thus $\tilde{B}_{\infty^{-a l g e b r a s}}\left(C^{*}(X), d_{C^{*}(X)}, \smile,\left\{E_{1, k}^{X}\right\},\left\{F_{p, q}^{X}\right\}\right)$ and $\left(C^{*}(Y), d_{C^{*}(Y)}, \smile,\left\{E_{1, k}^{Y}\right\},\left\{F_{p, q}^{Y}\right\}\right)$ are weakly equivalent.

Conversely, suppose that the $\tilde{B}_{\infty^{-}}$-algebras $\left(C^{*}(X), d_{C^{*}(X)}, \smile,\left\{E_{1, k}^{X}\right\},\left\{F_{p, q}^{X}\right\}\right)$ and $\left(C^{*}(Y), d_{C^{*}(Y)}, \smile,\left\{E_{1, k}^{Y}\right\},\left\{F_{p, q}^{Y}\right\}\right)$ are weakly equivalent. Again, as above, we assume that there is a single 1-connected $\tilde{B}_{\infty}$-algebra $\left(V, d_{V}, \cdot,\left\{E_{p, q}^{V}\right\},\left\{F_{p, q}^{V}\right\}\right)$ connecting them

$$
\left(C^{*}(X), d_{C^{*}(X)}, \smile,\left\{E_{1, k}^{X}\right\}\right) \stackrel{f_{1}}{\longleftarrow}\left(V, d_{V}, \cdot,\left\{E_{p, q}^{V}\right\}\right) \stackrel{f_{2}}{\rightarrow}\left(C^{*}(Y), d_{C^{*}(Y)}, \smile,\left\{E_{1, k}^{Y}\right\}\right) .
$$

From Subsection 3.2 we know that on the homology $H C^{*} X$ of the space $X$ there exist a minimal commutative $A_{\infty}$-algebra structure $\left(H C^{*} X,\left\{m_{i}\right\}\right)$ and the following maps in both directions

$$
B f:\left(B H C^{*} X, d_{m}, \Delta, \mu_{s h}\right) \rightarrow\left(B C^{*} X, d_{B C^{*} X}, \Delta, \mu_{s h}\right)
$$

and

$$
B g:\left(B C^{*} X, d_{B C^{*} X}, \Delta, \mu_{s h}\right) \rightarrow\left(B H C^{*} X, d_{m}, \Delta, \mu_{s h}\right)
$$

the first map is strictly multiplicative and the second is multiplicative up to homotopy. Similarly for space $Y$.

On the other hand, since $\left(V, d_{V}, \cdot,\left\{E_{p, q}^{V}\right\},\left\{F_{p, q}^{V}\right\}\right)$ is a $\tilde{B}_{\infty}$-algebra the Bar construction $\left(B V, d_{B V}, \Delta, \mu_{E^{V}}\right)$ is a $H a h$. Then by Proposition 47 there is a commutative and associative product $\tilde{\mu}_{E^{V}}$ on $B V$ such that $\tilde{\mu}_{E^{V}} \sim \mu_{E^{V}}$; this implies that the identity map

$$
1:\left(B V, d_{B V}, \Delta, \tilde{\mu}_{E^{V}}\right) \rightarrow\left(B V, d_{B V}, \Delta, \mu_{E^{V}}\right)
$$

is multiplicative up to homotopy.

By the same proposition we know that the resulting Hopf algebra $\left(B V, d_{B V}, \Delta, \tilde{\mu}_{E^{V}}\right)$ (now with commutative and associative multiplication) has the form

$$
\left(B V, d_{B V}, \Delta, \tilde{\mu}_{E^{V}}\right)=\left(U^{c} \mathcal{L}^{\mathrm{c}} \mathrm{W}, \mathrm{d}, \Delta_{\mathcal{L}^{\mathrm{c}} \mathrm{W}}, \mu_{\mathcal{L}^{\mathrm{c}} \mathrm{W}}\right)
$$


for some $Q$-module $W$ that is isomorphic to the $Q$-module $V$. But the latter is canonically isomorphic to $\left(B W, d_{B W}, \Delta, \mu_{s h}\right)$. Then by Theorem 24 on homology $H(W)$ there exists a minimal commutative $A_{\infty}$-algebra structure $\left(H(W),\left\{m_{i}\right\}\right)$ and quasi-isomorphisms in both directions

$$
B f:\left(B H(W), d_{m}, \Delta, \mu_{s h}\right) \rightarrow\left(B W, d_{B W}, \Delta, \mu_{s h}\right)
$$

and

$$
B g:\left(B W, d_{B W}, \Delta, \mu_{s h}\right) \rightarrow\left(B H(W), d_{m}, \Delta, \mu_{s h}\right)
$$

Therefore the entire composition

$$
\left(B H(W), \mu_{s h}\right) \stackrel{B f}{\rightarrow}\left(B W, \mu_{s h}\right)=\left(U^{c} \mathcal{L}^{\mathrm{c}} \mathrm{W}, \mu_{\mathcal{L}^{\mathrm{c}} \mathrm{W}}\right)=\left(\mathrm{BV}, \tilde{\mu}_{\mathrm{Ev}}\right) \stackrel{1}{\rightarrow}\left(\mathrm{BV}, \mu_{\mathrm{EV}}\right)
$$

is multiplicative up to homotopy. Thus we have the following diagram

$$
\begin{aligned}
& \left(B H C^{*}(X), \mu_{s h}\right) \quad\left(B H(W), \mu_{s h}\right) \quad\left(B H C^{*}(Y), \mu_{s h}\right) \\
& \uparrow B g_{X} \quad \downarrow B f \quad \uparrow B g_{Y} \\
& \left(B C^{*}(X), \mu_{E^{X}}\right) \stackrel{B f_{1}}{\longleftarrow} \quad\left(B V, \mu_{E^{V}}\right) \quad \stackrel{B f_{2}}{\longrightarrow}\left(B C^{*}(Y), \mu_{E^{Y}}\right)
\end{aligned}
$$

of the quasi-isomorphisms in $H A H$; then the compositions

$$
F_{1}=B g_{X} B f_{1} B f:\left(B H(W), d_{m}, \mu_{s h}, \Delta\right) \rightarrow\left(B H C^{*}(X), d_{m}, \mu_{s h}, \Delta\right)
$$

and

$$
F_{2}=B g_{Y} B f_{2} B f:\left(B H(W), d_{m}, \mu_{s h}, \Delta\right) \rightarrow\left(B H C^{*}(Y), d_{m}, \mu_{s h}, \Delta\right)
$$

are quasi-isomorphism too. But quasi-isomorphisms of the Bar-constructions over minimal $A_{\infty}$-algebras are isomorphisms. By Proposition 48, without loss of generality, we can assume that $F_{1}$ and $F_{2}$ are $D G$-Hopf algebra maps. Then the composition

$$
F_{2} F_{1}^{-1}:\left(B H C^{*}(X), d_{m}^{X}, \mu_{s h}, \Delta\right) \rightarrow\left(B H C^{*}(Y), d_{m}^{Y}, \mu_{s h}, \Delta\right)
$$

is an isomorphism of $D G$-Hopf algebras. This implies that the underlying $A_{\infty}$-algebra morphism

$$
P_{1} F_{2} F_{1}^{-1}:\left(H C^{*}(X),\left\{m_{i}^{X}\right\}\right) \rightarrow\left(H C^{*}(Y),\left\{m_{i}^{Y}\right\}\right)
$$


is an isomorphism in $C$ - $A_{\infty}-A l g$. Then by Theorem 73 the commutative $A_{\infty}$-algebras $\left(H A^{*}(X),\left\{m_{i}^{X}\right\}\right)$ and $\left(H A^{*}(Y),\left\{m_{i}^{Y}\right\}\right)$ are isomorphic too. The latter implies that $X$ and $Y$ have the same rational homotopy type; see the second bijection in the introduction.

We know that the correspondence $X \mapsto\left(C^{*}(X), d_{C^{*}(X)}, \smile,\left\{E_{1, k}^{X}\right\},\left\{F_{p, q}^{X}\right\}\right)$ is natural (see Section 3) and so it defines a transformation of the following sets

$$
\begin{aligned}
& \text { \{rational homotopy types }\} \rightarrow \text { \{weak equivalent classes of } \\
& \text { 1-connected } \left.\tilde{B}_{\infty} \text { - algebras }\right\} \text {; }
\end{aligned}
$$

the correctness and injectivity of this correspondence is provided by Theorem 74 . Let us assume now that 1-reduced spaces $X$ and 1-connected $\tilde{B}_{\infty}$-algebras $\left(V,\left\{m_{i}\right\},\left\{E_{p, q}\right\}\right)$ are such that $H^{*}(X, Q)$ and $H^{*}(V, Q)$ have finite type. Then

THEOREM 75. The sets

$$
\begin{aligned}
\{\text { rational homotopy types }\} \simeq & \{\text { weak equivalence classes of } \\
& \left.1 \text {-connected } \tilde{B}_{\infty} \text {-algebras }\right\}
\end{aligned}
$$

are bijective.

Proof. Let $\left(V,\left\{m_{i}\right\},\left\{E_{p, q}\right\}\right)$ be a $\tilde{B}_{\infty}$-algebra. We want to show that there exists a space $X$ such that $\left(C^{*}(X), d_{C^{*}(X)}, \smile,\left\{E_{1, k}^{X}\right\}\right)$ and $\left(V,\left\{m_{i}\right\},\left\{E_{p, q}\right\}\right)$ are weakly equivalent $\tilde{B}_{\infty}$-algebras.

Since $\left(B V, d_{m}^{B V}, \Delta, \mu_{E}\right) \in H A H$ we know that there exists a $Q$-module $W$ such that

$$
B f:\left(B H(W), d, \Delta, \mu_{s h}\right) \rightarrow\left(B V, d_{m}^{B V}, \Delta, \mu_{E}\right)
$$

is a quasi-isomorphism in $H A H$ (in more detail we have described this map in the proof of Theorem 74). By construction, the differential $d$ on $B H(W)$ defines a minimal commutative $A_{\infty}$-algebra structure $\left\{m_{i}\right\}=\left\{P_{1} d\right\}$ on $H(W)$, then by the second bijection of the introduction there exists a space $X$ such that $\left(H A^{*}(X),\left\{m_{i}^{X}\right\}\right)$ and 
$\left(H(W),\left\{m_{i}\right\}\right)$ are isomorphic in $C-A_{\infty}-A l g$; i.e there arises a $D G$ Hopf algebra isomorphism

$$
G:\left(B H A^{*}(X), d_{m}^{B H^{*} X}, \Delta, \mu_{s h}\right) \rightarrow\left(B H(W), d, \Delta, \mu_{s h}\right)
$$

of appropriate Bar constructions.

We show below that for this particular $X$ the $\tilde{B}_{\infty}$-algebras $\left(V,\left\{m_{i}\right\},\left\{E_{p, q}\right\}\right)$ and $\left(C^{*}(X), d_{C^{*}(X)}, \smile,\left\{E_{1, k}\right\}\right)$ are weakly equivalent.

Note that we have two quasi-isomorphisms in $H A H$ : first, the composition

$$
B f G:\left(B H A^{*}(X), d_{m}^{B H^{*} X}, \Delta, \mu_{s h}\right) \rightarrow\left(B V, d_{m}^{B V}, \Delta, \mu_{E}\right),
$$

and the second,

$$
\begin{gathered}
\beta^{-1} B f^{\prime}:\left(B H C^{*}(X), d_{m}^{B H^{*} X}, \Delta, \mu_{s h}\right) \rightarrow\left(B C^{*}(X), d_{m}^{B C^{*} X}, \Delta, \mu_{s h}\right) \rightarrow \\
\rightarrow\left(B C^{*}(X), d_{B C^{*}(X)}, \Delta, \mu_{E}\right)
\end{gathered}
$$

where $\beta^{-1}$ is the isomorphism of Theorem 69 , and $B f^{\prime}$ is a coalgebra map induced by the $A_{\infty}$-algebra morphism $\left\{f_{i}^{\prime}\right\}$ of Proposition 72 . Then the diagram

$$
\begin{array}{cc}
\left(B H C^{*}(X), d_{m}^{B H^{*} X}, \Delta, \mu_{s h}\right) & \stackrel{\Sigma}{\cong}\left(B H A^{*}(X), d_{m}^{B H^{*} X}, \Delta, \mu_{s h}\right) \\
\beta^{-1} B f^{\prime} \downarrow & \downarrow^{B f G} \\
\left(B C^{*}(X), d_{B C^{*}(X)}, \Delta, \mu_{E}\right) & \left(B V, d_{m}^{B V}, \Delta, \mu_{E}\right)
\end{array}
$$

gives a desired chain of underlying $\tilde{B}_{\infty}$-algebras and their quasi-isomorphisms

$$
\begin{array}{ccc}
\left(H C^{*}(X),\left\{m_{i}^{H C^{*}(X)}\right\}\right) & \stackrel{P_{1} \Sigma}{\cong} & \left(H A^{*}(X),\left\{m_{i}^{H A^{*}(X)}\right\}\right) \\
P_{1} \beta^{-1} B f^{\prime} \downarrow & \downarrow^{P_{1} B f G} \\
\left(C^{*}(X), d_{C^{*}(X)}, \smile,\left\{E_{1, k}\right\}\right) & \left(V,\left\{m_{i}\right\},\left\{E_{p, q}\right\}\right)
\end{array}
$$

where $P_{1} \Sigma$ is the isomorphism of Theorem 73 ; i.e. $\left(C^{*}(X), d_{C^{*}(X)}, \smile,\left\{E_{1, k}^{X}\right\}\right)$ and $\left(V,\left\{m_{i}\right\},\left\{E_{p, q}\right\}\right)$ are weakly equivalent in $\tilde{B}_{\infty}-A l g$ as desired. 
Let $X$ and $Y$ be simply connected $C W$ complexes and $f: X \rightarrow Y$ be any pointpreserving continuous map. We know that the singular cochain functor induces a $\tilde{B}_{\infty}$-algebra morphism

$$
C^{*} f:\left(C^{*}(Y), d_{C^{*}(Y)}, \smile,\left\{E_{1, k}^{Y}\right\},\left\{F_{p, q}^{Y}\right\}\right) \rightarrow\left(C^{*}(X), d_{C^{*}(X)}, \smile,\left\{E_{1, k}^{X}\right\},\left\{F_{p, q}^{X}\right\}\right)
$$

THEOREM 76. The singular cochain functor induces a bijection of the following sets

$$
\begin{aligned}
& \left\{Q \text { -locally homotopy classes } \simeq \left\{\text { homotopy classes of } \tilde{B}_{\infty}\right.\right. \text {-alg- } \\
& \text { of continuous maps } \left.(X, Y)\} \quad \text { ebra morphisms }\left(C^{*} Y, C^{*} X\right)\right\}
\end{aligned}
$$

PROOF. Let us show first that this correspondence is well defined and is an injection, which is equivalent to say that $f_{1}, f_{2}: X \rightarrow Y$ are $Q$-locally homotopic maps if and only if $C^{*} f_{1}, C^{*} f_{2}: C^{*} Y \rightarrow C^{*} X$ are homotopic in $\tilde{B}_{\infty}-A l g$.

The continuous maps $f_{1}, f_{2}: X \rightarrow Y$ are $Q$-locally homotopic if and only if the Lie coalgebra maps $\mathcal{L}^{c} f_{1}, \mathcal{L}^{c} f_{2}: \mathcal{L}^{c}\left(s^{-1} I C^{*}(Y)\right) \rightarrow \mathcal{L}^{c}\left(s^{-1} I C^{*}(X)\right)$ are homotopic in $D L C$, see Remark 71 . We know that $\mathcal{L}^{c} f_{1}, \mathcal{L}^{c} f_{2}$ are homotopic if and only if

$$
U^{c} \mathcal{L}^{c} f_{1}, U^{c} \mathcal{L}^{c} f_{2}: U^{c} \mathcal{L}^{c}\left(s^{-1} I C^{*}(Y)\right) \rightarrow U^{c} \mathcal{L}^{c}\left(s^{-1} I C^{*}(X)\right)
$$

are homotopic in $H A H$.

On the other hand each of the continuous maps $f_{1}, f_{2}: X \rightarrow Y$ induce $H A H$ morphisms

$$
B C^{*} f_{1}, B C^{*} f_{2}:\left(B C^{*}(Y), d_{B C^{*} Y}, \Delta, \mu_{E^{Y}}\right) \rightarrow\left(B C^{*}(X), d_{B C^{*} X}, \Delta, \mu_{E^{X}}\right)
$$

We know that $\left(B C^{*}(X), d_{B C^{*} X}, \Delta, \mu_{E^{X}}\right)$ and $U^{c} \mathcal{L}^{c}\left(s^{-1} I C^{*}(X)\right.$ are naturally isomorphic in $H A H$. This isomorphism is obtained by first constructing the strictly commutative and associative product $\mu_{\tilde{E}^{x}}$ on $\left(B C^{*}(X)\right.$ (Theorem 68) and then composing the resulting $D G$ Hopf algebra $\left(B C^{*}(X), d_{B C^{*} X}, \Delta, \mu_{\tilde{E}^{X}}\right)$ with $D G$ Hopf algebra $U^{c} \mathcal{L}^{c}\left(s^{-1} I C^{*}(X)\right.$ via the natural Hopf algebra isomorphism $\beta$ of Theorem 69 . Analogously $\left(B C^{*}(Y), d_{B C^{*} Y}, \Delta, \mu_{E^{Y}}\right)$ is isomorphic to $U^{c} \mathcal{L}^{c}\left(s^{-1} I C^{*}(Y)\right.$. Clearly, by construction $U^{c} \mathcal{L}^{c} f_{1}, U^{c} \mathcal{L}^{c} f_{2}$ are homotopic in $H A H$ if and only if $B C^{*} f_{1}, B C^{*} f_{2}$ 
are homotopic in $H A H$. Finally, by Proposition $66 B C^{*} f_{1}, B C^{*} f_{2}$ are homotopic in $H A H$ if and only if the underlying $\tilde{B}_{\infty}$-algebra morphisms $C^{*} f_{1}, C^{*} f_{2}$ are homotopic in the $\tilde{B}_{\infty}-A l g$ category as desired.

Thus it remains to show that this correspondence is a surjection, i.e. for any $\tilde{B}_{\infty}$-algebra homomorphism

$$
g:\left(C^{*}(Y), d_{C^{*}(Y)}, \smile,\left\{E_{1, k}^{Y}\right\},\left\{F_{p, q}^{Y}\right\}\right) \rightarrow\left(C^{*}(X), d_{C^{*}(X)}, \smile,\left\{E_{1, k}^{X}\right\},\left\{F_{p, q}^{X}\right\}\right)
$$

there exists a continuous map $f: X \rightarrow Y$ such that $C^{*} f \sim g \in \tilde{B}_{\infty}-A l g$. Given $\tilde{B}_{\infty}$-algebra morphism $g$ induces a $H A H$ homomorphism

$$
B g:\left(B C^{*}(Y), d_{B C^{*} Y}, \Delta, \mu_{E}\right) \rightarrow\left(B C^{*}(X), d_{B C^{*} X}, \Delta, \mu_{E}\right)
$$

We know that $\left(B C^{*}(Y), d_{B C^{*} Y}, \Delta, \mu_{E}\right)$ and $\left(B C^{*}(X), d_{B C^{*} X}, \Delta, \mu_{E}\right)$ are naturally isomorphic in $H A H$ to $U^{c} \mathcal{L}^{c}\left(s^{-1} I C^{*}(Y)\right.$ and $U^{c} \mathcal{L}^{c}\left(s^{-1} I C^{*}(X)\right.$ respectively, through which they are identified. Then by Proposition $48 \mathrm{Bg}$ is homotopic to a $D G$-Hopf algebra map $U^{c} \alpha, B g \sim U^{c} \alpha \in H A H$, for some $D G$-Lie coalgebra homomorphism

$$
\alpha: \mathcal{L}^{c}\left(s^{-1} I C^{*}(Y)\right) \rightarrow \mathcal{L}^{c}\left(s^{-1} I C^{*}(X)\right)
$$

(compare with Theorem 44). By Remark 71 for $\alpha$ there exists a continuous map $f: X \rightarrow Y$ such that $\mathcal{L}^{c}\left(s^{-1} I C^{*} f\right) \sim \alpha \in D L C$ and hence $U^{c} \mathcal{L}^{c}\left(s^{-1} I C^{*} f\right) \sim$ $U^{c} \alpha \in H A H$. Therefore $U^{c} \mathcal{L}^{c}\left(s^{-1} I C^{*} f\right) \sim B g \in H A H$. But by construction $U^{c} \mathcal{L}^{c}\left(s^{-1} I C^{*} f\right) \sim B C^{*} f \in H A H$, which implies that $B C^{*} f \sim B g \in H A H$. It follows now from Definition 65 that $C^{*} f \sim g \in \tilde{B}_{\infty^{-}} A l g$. 


\section{BIBLIOGRAPHY}

[1] M. Andre, Hopf algebras with divided powers, J. Algebra 18 (1971), 19-50.

[2] J.F. Adams, On the cobar construction, Prof. nat. Acad. Sci. (USA) 42 (1956), 409-412.

[3] D.J. Anick, Hopf algebras up to homotopy, Journal of The AMS, 2 (1989), 417-453.

[4] D.J. Anick, R-local homotopy thory, Lecture Notes in Math., vo. 1418, (1990), 78-85.

[5] M. Aubry and J.-M. Lemaire, Homotopies d'algebres de Lie et de leurs algebtres enveloppantes, Algebraic Topology-Rational Homotopy, Lecture Notes in Math., no 1318, Springer-Verlag, Berlin and New York, 1988, 26-30.

[6] H.J. Baues, The double bar and cobar construction, Compositio Math. 43 (1981), 331-341.

[7] H.J. Baues, The cobar construction as a Hopf algebra, Invent. Math., 132 (1998), 467-489.

[8] H.J. Baues and J.M. Lemaire, Minimal models in homotopy theory, Math. Ann. 225, (1977), 219-242.

[9] N.A. Berikashvili, The homology theory of spaces, Bull. of Georg. Acad. Sci., 59 (1970), 13-16.

[10] N.A. Berikashvili, On the differentials of spectral sequence, Proc. Tbil. Math. Inst., 51 (1976), $1-105$.

[11] E.H. Brown, Twisted tensor products, Ann. of Math. 69 (1959), 223-246.

[12] R. Brown, the twisted Eilenberg-Zilber theorem, Celebrazioni Archimedee del secolo XX, Simposio di topologia, (1967), 34-37.

[13] G. de Rham, Sur l'analysis situs des varietes a n dimensions, J. Math. Pures Appl. 10(1931), $115-200$.

[14] S. Eilenberg and S. MacLane, Acyclic Models, Amer. J. Math 75 (1953) 189-199.

[15] Y. Felix, S. Halperin and J-C. Thomas, Rational homotopy theory, Preprint, 1998.

[16] E. Friedlander, P.A. Griffiths and J. Morgan, Homotopy theory and differential forms, Mimeographed notes, Seminar of Geometry, Florence, 1972.

[17] M. Gerstenhaber and A.A. Voronov, Higher operations on Hochschild complex, Functional Analysis and its Applications, 29 (1995), 1-5. 
[18] M. Gerstenhaber and A.A. Voronov, Homotopy Gerstenhaber algebras and moduli space operad, IMNR International Math. Research Notices NO 3, 141-153 (1995).

[19] E. Getzler and J.D. Jones, Operads, homotopy algebra, and iterated integrals for double loop spaces, Preprint, 1995.

[20] V.K.A.M. Gugenheim, On the multiplicative structure of the De Rham theory, Journal of Differential Geometry, Vol. 11, No. 2 (1976), 309-314.

[21] V.K.A.M. Gugenheim, L. Lambe and J.D. Stasheff, Perturbation theory in differential homological algebra II, Illinois J. Math. 35 (1991), 357-373.

[22] J. Huebshmann and T. Kadeishvili, Small models for chain algebras, Math. Zeitschrift, 207 (1991), 245-280.

[23] T.V. Kadeishvili, On the homology theory of fiber spaces, Russian Math. Surveys, 35:3 (1980), 231-238.

[24] T.V Kadeishvili, $A(\infty)$-algebra structure in cohomology and rational homotopy type, Forschungsschwerpunkt Geometrie, Universitat Heidelberg, Math. Inst. Heft 37, 1-64, Oktober 1988 .

[25] T.V. Kadeishvili, $A(\infty)$-algebra structure in cohomology and rational homotopy type, Proc. A. Razmadze Math. Inst., 107, 1993, 1-94.

[26] T.V. Kadeishvili, The category of differential coalgebras and the category of $A_{\infty}$-algebras, Proc. A. Razmadze Math. Inst. 1985.

[27] T.V. Kadeishvili, The predifferential of a twisting product, Russian Math. Surveys 41:6 (1986), 135-147.

[28] T.V. Kadeishvili and S. Saneblidze, A cubical model for fibration, Math. AT/0210006.

[29] Z. Kharebava, On a multiplicativity up to homotopy of the Gugenheim map, Georgian Math. Journal 9 (2002), No 3, 549-566.

[30] L.G. Khelaia, On some chain operations, Bull. of Georg. Acad. Sci., V. 96, No. 3 (1979), 532-535.

[31] T. Lada, Commutators of $A_{\infty}$ structures, Contemporary Mathematics, V. 227, (1999), 227-233.

[32] D. Lemann, Théorie homotopique des forms différentielles (d'aprés D. Sullivan). -Société mathématique de France, asterisque 45, 1977.

[33] M. Markl, A cohomology theory for $A(M)$-algebras and applications, Journ. Pure Appl. Algebra 83 (1992), 141-175.

[34] J.P. May, Simplicial objects in algebraic topology, Van. Nostrand, New York, 1967.

[35] W. Michaelis, Lie Coalgebras, Advances in Mathematics, 38 (1980), 1-54. 
[36] J. Milnor and J.C. Moore, On the structure of Hopf algebras, Ann. of Math. 81, 211-264 (1965).

[37] H.J. Munkholm, The Eilenberg-Moore spectral sequence and strongly homotopy multiplicative maps, Journal Pure and Application Algebra, 5 (1974), 1-50.

[38] D. Quillen, Rational homotopy theory, Annals of Math. 90 (1969), 205-295.

[39] V.A. Smirnov, Homology theory of fibre spaces, Russian Math. Suveys, 35:3 (1980), 183-188.

[40] E. H. Spanier, Algebraic topology, McGraw-Hill series in higher mathematics, McGraw-Hill, Academic Press, 1966.

[41] J.D. Stasheff, Homotopy associativity of $H$-spaces, I, II, Trans. Amer. Math. Soc., 108 (1963), 275-312.

[42] J. Stasheff and H. Halperin, Differential homological algebra in its own rite, Proc. Advanced Study Inst. Algebraic Topology, Aarhus, 1970.

[43] D. Sullivan, Infinitisimal computations in topology. Publ. I.H.E.S. 47 (1977), 269-331.

[44] D. Tanre, Homotopie rationnelle: Modeles de Chen, quillen, Sullivan, Lecture Notes in Math., no 1025, Springer-Verlag, Berlin and New York, 1983. 